\title{
Thermal fluctuations, quenched disorder, phase transitions, and transport in type-II superconductors
}

\author{
Daniel S. Fisher* \\ Physics Department, Princeton University, Princeton, New Jersey 08544 \\ Matthew P. A. Fisher \\ IBM T. J. Watson Research Center, P.O. Box 218, Yorktown Heights, New York 10598 \\ David A. Huse \\ AT\&T Bell Laboratories, Murray Hill, New Jersey 07974 \\ (Received 28 February 1990)
}

\begin{abstract}
The effects of thermal fluctuations, quenched disorder, and anisotropy on the phases and phase transitions in type-II superconductors are examined, focusing on linear and nonlinear transport properties. In zero magnetic field there are two crossovers upon approaching $T_{c}$, first the "Ginzburg" crossover from mean-field behavior to the universality class of an uncharged superfluid, and then, much closer to $T_{c}$ for strongly type-II systems, a crossover to the universality class of a charged superfluid. The primary focus of this paper is on the behavior in the presence of a penetrating magnetic field. In a clean system the vortex-lattice phase can melt due to thermal fluctuations; we estimate the phase boundary in a variety of regimes. Pinning of vortices due to impurities or other defects destroys the long-range correlations of the vortex lattice, probably replacing it with a new vortex-glass phase that has spin-glasslike off-diagonal long-range order and is truly superconducting, in contrast to conventional theories of "flux creep." The properties of this vortex-glass phase are examined, as well as the critical behavior near the transition from the vortex-glass to the vortex-fluid phase. The crossover from lattice to vortex-glass behavior for weak pinning is also examined. Linear and nonlinear conductivity measurements and other experiments on the high- $T_{c}$ superconductors $\mathrm{Y}-\mathrm{Ba}-\mathrm{Cu}-\mathrm{O}$ and $\mathrm{Bi}-\mathrm{Sr}-\mathrm{Ca}-\mathrm{Cu}-\mathrm{O}$ are discussed in light of the results. The latter is found to exhibit strongly two-dimensional behavior over large portions of its phase diagram.
\end{abstract}

\section{INTRODUCTION AND SUMMARY}

In conventional ("low- $T_{c}$ ") bulk superconductors, fluctuation effects are generally quite small. This is because the length,

$$
\Lambda_{T}=\frac{\phi_{0}^{2}}{16 \pi^{2} T} \simeq \frac{2 \times 10^{8} \AA \mathrm{K}}{T},
$$

set by the flux quantum, $\phi_{0}=h c / 2 e$, and the temperature, $T$, is much larger than any other characteristic lengths, except extremely close to $T_{c}$. Because fluctuation effects are weak, the behavior as a function of temperature, field, current, etc., is generally quite close to that found in mean-field theory. ${ }^{1,2}$ In particular, the onset of dissipation with increasing current or temperature is rather sharp, yielding fairly well-defined critical currents for temperatures $T$ less than $T_{c}$, and a sharp transition in the linear resistivity as $T$ passes through $T_{c}$.

Nevertheless, there is an important question of principle: do fluctuations destroy the defining property of superconductors - a vanishing linear resistivity? In the Meissner phase, the answer is certainly no. However, in a type-II superconductor at magnetic fields above $H_{c 1}$ where flux penetrates, this question has never been satisfactorily answered, although the standard Anderson-Kim theory of "flux creep" $3-5$ would answer the question in the affirmative. For conventional superconductors (with the exception of thin films, long wires, or granular materials) this is largely an academic question. In the high$T_{c}$ cuprate superconductors, on the other hand, a combination of not completely unrelated factors-(i) higher temperatures, (ii) shorter coherence length $\xi$, (iii) large magnetic penetration length $\lambda$, and (iv) quasi-twodimensionality-conspire to drastically enhance the effects of thermal fluctuations (see, for example, Refs. 6 and 7) and make this question far more interesting. In this paper we investigate various consequences of thermal fluctuations and the crucial role played by quenched disorder. We will focus on the qualitative behavior of the various phases of a type-II superconductor and the behavior near to the phase transitions between them. The effects of quasi-two-dimensionality will often play a large role and we examine various two- and three-dimensional regimes.

The mean-field phase diagram ${ }^{1}$ of a type-II superconductor is shown in Fig. 1. Of the two ordered phases, the Meissner phase is, in all respects, the more stable against thermal fluctuations and quenched disorder. Thus, most of this paper focuses on the behavior in the presence of a penetrating magnetic field, $H>H_{c 1}$. The Abrikosov vortex lattice phase ${ }^{1}$ which occurs in this regime has two spontaneously broken symmetries: First, as in the Meiss- 


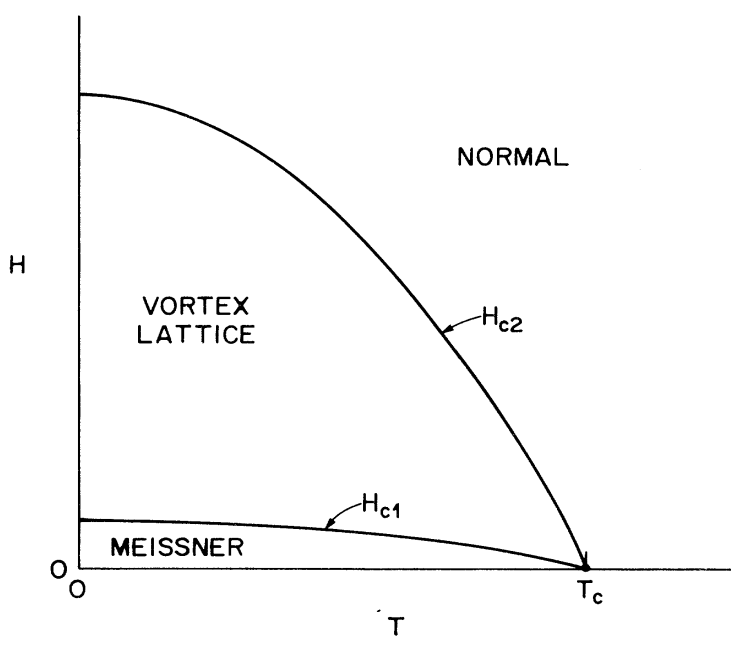

FIG. 1. Mean-field phase diagram of a type-II superconductor (schematic) as a function of applied magnetic field $H$ and temperature $T$.

ner phase, there is phase coherence (off-diagonal, longrange order) in the pairing field, albeit with a nontrivial spatial structure reflecting the vortex lattice. Second, there is crystalline long-range order of the vortex lattice that breaks the translational symmetry. In the absence of vortex pinning, this phase is not truly superconducting: it will have a nonzero Ohmic resistivity due to vortex (flux) flow, ${ }^{8}$ except possibly for currents strictly parallel to the vortices.

In the presence of vortex pinning due to disorder or other imperfections, vortex flow is impeded. However, Larkin and Ovchinikov ${ }^{9}$ have shown that, in equilibrium, the crystalline long-range order of the vortex lattice phase is unstable against the introduction of random pinning for spatial dimensionality $D$ less than four. Thus, real two- or three-dimensional systems, provided they are in equilibrium, cannot have a long-range-ordered vortex lattice in the presence of quenched disorder. Existing theories of vortex pinning ${ }^{3-5}$ appear to have generally neglected the question of long-range phase coherence beyond the length scale where the lattice correlations are destroyed. We will argue that a vortex-glass phase may occur with vanishing resistivity and long-range phase coherence, again with a nontrivial spatial structure reflecting the positions of the (now randomly pinned) vortices. ${ }^{10-12}$ This ordering into a specific nontrivial arrangement determined by the particular details of the quenched disorder in the system is very analogous to the magnetic order that occurs in a spin glass, ${ }^{13,14}$ thus, the name vortex glass. For a three-dimensional type-II superconductor it appears quite possible that such a vortex-glass phase can be stable at nonzero temperature, yielding a phase diagram like Fig. 2. For a twodimensional (2D) superconductor, on the other hand, we expect, by analogy to two-dimensional spin glasses, ${ }^{13,14}$ the vortex-glass long-range order to be unstable against thermal fluctuations at arbitrarily low temperatures-

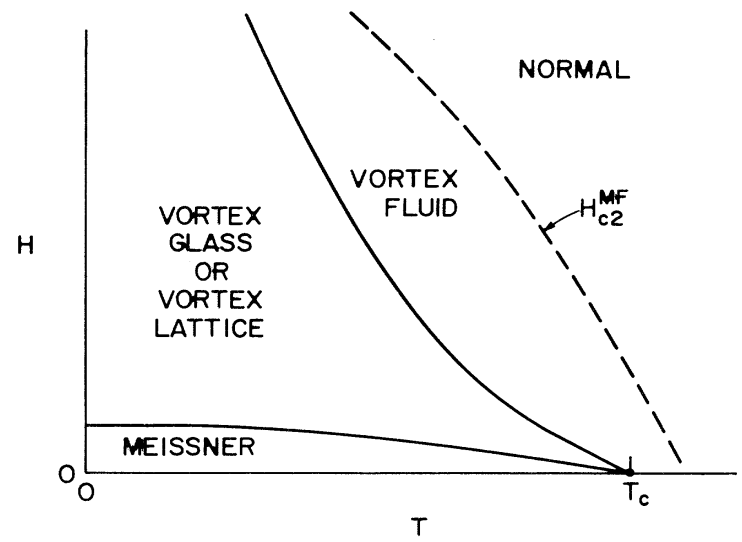

FIG. 2. Schematic phase diagram of a three-dimensional type-II superconductor with strong thermal fluctuations. The crossover from the normal regime to the vortex-fluid regime is not a sharp phase transition and occurs near the location of the mean-field transition $H_{c 2}^{\mathrm{MF}}$. Without random pinning, a vortexlattice phase is present and the vortex-fluid phase also intrudes at $H_{c 1}$ between the Meissner and vortex-lattice phases. This intrusion is not shown here since, for parameters appropriate to the cuprate superconductors, it occurs over too narrow an interval of $H$ to be seen in this figure (see Sec. V and Fig. 4). With random pinning we expect the vortex-lattice phase to be replaced with a vortex-glass phase.

and the system thus not truly superconducting for any $T>0$. The properties of the vortex-glass phase have been discussed briefly by one of us ${ }^{11}$ and are discussed in more detail below in Sec. VIII.

As the temperature is raised, increased thermal fluctuations will cause the vortex lattice and vortex-glass phases to disorder (melt) into a vortex fluid phase. ${ }^{6,7,15-18}$ In the cuprate superconductors this transition can occur at a temperature well below the meanfield transition $H_{c 2}^{\mathrm{MF}}$; see Fig. 2. Much of the ordering energy still comes out at $H_{c 2}^{\mathrm{MF}}$, however, so one expects the specific heat to show a smooth maximum near there. For zero or weak disorder, the melting transition may be of first order, in which case there will be a small latent heat of melting that would, at least in principle, be observable in the specific heat. The vortex fluid phase is a fully disordered phase and is thus not separated from the normal phase by a true phase transition - they really are the same phase and should be connected by a smooth crossover. In the normal phase there is essentially no local pairing at all so one cannot usefully describe the system in terms of vortex positions. Below $H_{c 2}^{\mathrm{MF}}$, on the other hand, there is a local tendency toward pairing, but in the vortex fluid phase the pairing field is strongly fluctuating with only a finite, although large, correlation length. In this regime the system thus may be described in terms of mobile, fluctuating vortices, as in the usual analysis of superfluid or superconducting films just above the Kosterlitz-Thouless transition. ${ }^{19}$ As the system is cooled further the correlation length of the pairing field grows 
and diverges when the system orders into either a vortex-glass or vortex-lattice phase, depending on whether or not random pinning is present. In bulk low- $T_{c}$ superconductors, vortex-lattice or vortex-glass melting essentially coincides with $H_{c 2}^{\mathrm{MF}}$, so that there is not an appreciable temperature range where a description in terms of a vortex fluid is appropriate.

For a two-dimensional superconductor in a magnetic field, a long-range-ordered vortex-glass phase is not expected except at $T=0$. However, the correlation length of the pairing field (i.e., the vortex-glass correlation length) should grow upon cooling and diverge as $T \rightarrow 0$. This is in contrast to the conventional "flux-creep" models $^{3-5}$ which assume that the longest relevant length scale is that over which lattice correlations persist, a length which remains finite as $T \rightarrow 0$. Although, in two dimensions, there will be only minor differences in the temperature dependence of the linear resistivity between these two models, the nonlinear $I-V$ characteristics differ markedly. The $2 \mathrm{D}$ vortex-glass scenario predicts a crossover current density scale $J_{X}$ separating Ohmic from non-Ohmic behavior, which varies as a power of temperature, $J_{X} \sim T^{1+v_{2}}$, with an exponent $v_{2}>0$, or perhaps even more rapidly with $T$, in contrast to the "flux-creep" models which predict a linear temperature dependence for $J_{X}$. This should be relevant in highly anisotropic high- $T_{c}$ superconductors which, as discussed below, behave as quasi-two-dimensional systems over a wide range of temperature and field.

With the exception of the direct imaging of flux lines at low magnetic fields, ${ }^{20}$ most experiments that study the magnetic properties of superconductors and their possible phase transitions are, in essence, transport measurements. ${ }^{21-24}$ Thus, in this paper we will emphasize results for both linear and nonlinear electrical transport.

Although this paper may be rather long, it is by no means intended to be complete - this problem has many aspects and different regimes and here we just touch on a few of them with the hope of raising more questions than we answer. An outline of the paper and summary of our results are as follows.

We begin in Sec. II with a review of the GinzburgLandau model for a type-II superconductor and its mean-field solution. ${ }^{1}$ This model is used throughout as the basis for studying the effects of fluctuations and disorder. This section also serves to establish much of the notation and define many of the parameters used later on.

In Sec. III we briefly discuss the Meissner phase. We examine the nonlinear resistivity due to thermally activated nucleation and growth of vortex loops. ${ }^{25}$ This leads to nonlinear dissipation and an electric field of the form

$$
E \sim \exp \left[-\left(J_{T} / J\right)^{\mu}\right]
$$

for small current density $J$. Here $\mu=1$ and the characteristic current scale $J_{T} \sim 1 / T$ at low temperatures. There is a second important characteristic current scale, $J_{F}$, above which the dissipation rapidly increases due to nonactivated phase-slip processes. Deep within the Meissner phase $J_{T} \gg J_{F}$, so that there is a sharp onset of dissipation at a "critical current" $J_{F}$, as depicted in Fig. 3(a). In the critical regime near $T_{c}$ (see below), however, $J_{T} \simeq J_{F}$ so that fluctuation effects should induce a smoother current-voltage characteristic, as illustrated in Fig. 3(b).

In Sec. IV we examine the normal-to-superconductor phase transition in zero magnetic field. This transition, at $T_{c} \equiv T_{c}(H=0)$, is significantly changed by the thermal fluctuations, which are relevant at the Gaussian fixed point governing the mean-field transition. The lowestorder fluctuation corrections to mean-field theory have received much attention in the past. ${ }^{2}$ The so-called "Ginzburg criterion" gives the point at which these fluctuation correlations become large upon approaching $T_{c}$ and for strongly type-II systems there is first a crossover to behavior governed by the $X Y$ model critical point of an unchanged superfluid. The Ginzburg criterion for a layered superconductor in the regime where interplane correlations are important takes the form
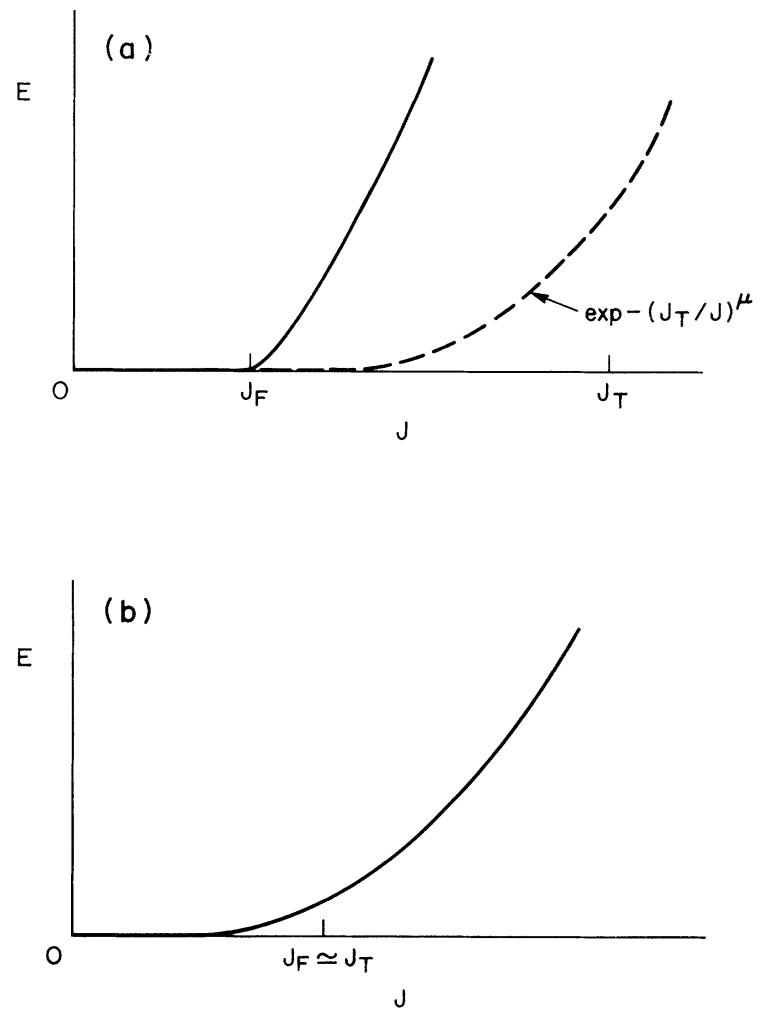

FIG. 3. Current-voltage characteristics in a superconducting (vortex-glass or Meissner) phase with $J$ the current density and $E$ the electric field in the sample. (a) In the mean-field regime there is a fairly sharp onset of nonactivated vortex flow and dissipation at current density $J_{F}$. The dissipation for $J \ll J_{F}$ is due to activated nucleation and growth of vortex-loop or bundle excitations and vanishes as $\exp \left[-\left(J_{T} / J\right)^{\mu}\right]$ for $J \rightarrow 0$. In the mean-field regime $J_{T} \gg J_{F}$, so the dissipation is still very small just below $J_{F}$. (b) In the thermal fluctuation-dominated critical regime, $J_{F}$ becomes of order $J_{T}$ so the onset of dissipation is smoother. 


$$
\lambda^{2} / \gamma \xi \simeq C \Lambda_{T}
$$

where $\Lambda_{T}$ is the thermal length defined in (1.1); $\lambda$ and $\xi$ are the in-plane penetration and coherence lengths, respectively; $C$ is a constant of order 1 and $\gamma=\xi_{z} / \xi_{\perp}$ is the anisotropy ratio between out-of-plane and in-plane coherence lengths. The cuprate superconductors are typically highly anisotropic $(\gamma<<1)$, strongly type II $(\kappa=\lambda / \xi \gg>1)$, and have a high $T_{c}$. All these features conspire to increase the role of fluctuations, making the fluctuation-dominated $X Y$ critical regime potentially accessible.

In the $X Y$ critical regime, the charge of the carriers and thus the coupling to the magnetic-field fluctuations is relevant, ${ }^{26}$ so that very close to $T_{c}$ one finally crosses over to the critical behavior of a charged superfluid thought to be in the universality class of an "inverted $X Y$ model."27 This last regime is very close to $T_{c}$ even in the high- $T_{c}$ systems, so we restrict our attention to the scaling in the intermediate ( $X Y$ model) regime. In this intermediate critical regime, the frequency-dependent conductivity in $D$ dimensions is argued to scale as

$$
\sigma(\omega) \approx \xi^{z+2-D} \mathcal{S}_{ \pm}\left(\omega \xi^{z}\right)
$$

while the nonlinear dc conductivity scales as ${ }^{28}$

$$
E(J) \approx J \xi^{D-2-z} \mathscr{E}_{ \pm}\left(J \xi^{D-1} \phi_{0} / c T\right)
$$

where $\xi$ is the coherence length which diverges at $T_{c}, z$ is the usual dynamic critical exponent, $E$ is the electric field and $J$ is the current density. The properties of the scaling functions $\mathcal{f}_{ \pm}$and $\mathscr{E}_{ \pm}$are elaborated below. We emphasize these scaling forms here because they should apply rather generally at continuous finite-temperature normal-to-superconductor phase transitions. Indeed (1.5) has already been used to experimentally address the issue of whether or not such a transition between a vortex-fluid and a vortex-glass superconductor occurs in the presence of quenched disorder and a penetrating magnetic field. ${ }^{22}$

In Sec. $V$ we investigate the vortex-lattice phase and the melting of the lattice, all in the absence of random pinning. In addition to existing below $H_{c 2}^{\mathrm{MF}}$ as shown in Fig. 2, the vortex-fluid phase intrudes, in a "reentrant" fashion, between the Meissner and vortex-lattice phases near $H_{c 1}$, as has been emphasized in recent work ${ }^{6,7,15}$ and is depicted in Fig. 4, where we show the low-field phase diagram in the $B-T$ plane. (Note, as $H \rightarrow H_{c 1}^{+}, B \rightarrow 0$.) It was initially ${ }^{6}$ suggested that the width of this vortexliquid phase is enhanced in proportion to the anisotropy of the hopping rate, which is quite large in some of the highly layered high- $T_{c}$ materials. However, near $H_{c 1}$ the magnetic interlayer coupling between vortices keeps the stiffness of an individual vortex-flux line large even in in the limit of no direct interlayer hopping (Josephson coupling).

At much higher fields, thermal fluctuations may also melt the lattice. The melting field $B_{M}(T)$, which may be well below $H_{c 2}^{\mathrm{MF}}$, can be estimated using the Lindemann criterion. ${ }^{6,7,16,17}$ The wavelength of the dominant inplane vortex fluctuations is of order $a_{v} \equiv\left(\phi_{0} / B\right)^{1 / 2}$, the vortex lattice constant, but a factor of the anisotropy ra-

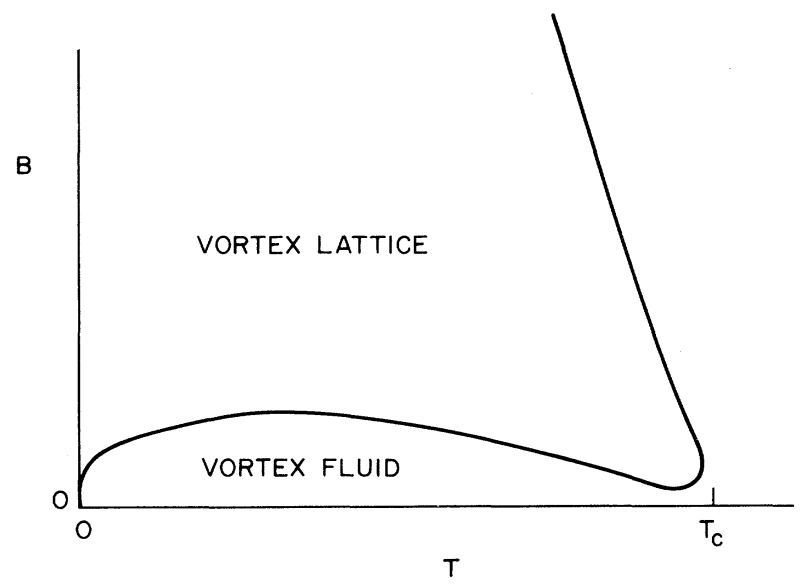

FIG. 4. Magnified view of the low- $B$ field, and thus $H$ near $H_{c 1}$, regime in Fig. 2 in the absence of quenched disorder or pinning. Note $B$ rather than $H$ is plotted, as is appropriate for a film geometry. The vortex-fluid phase actually intrudes between the Meissner $(B=0)$ and vortex-lattice phases down to arbitrarily low temperature. The width of this fluid phase in $B$ is of or$\operatorname{der} \phi_{0} / \lambda^{2}$.

tio, $\gamma$, smaller in the out-of-plane or $z$ direction. Thus, if the layer spacing $d$ exceeds $\gamma a_{v}$, the system's behavior is quasi-two-dimensional. A highly layered material like $\mathrm{Bi}_{2.2} \mathrm{Sr}_{2} \mathrm{Ca}_{0.8} \mathrm{Cu}_{2} \mathrm{O}_{8}$ (Bi-Sr-Ca-Cu-O), with ${ }^{29} \gamma \lesssim \frac{1}{50}$, is thus quasi-two-dimensional over much of the phase diagram, $B \gtrsim 3 \mathrm{kG}$. In this regime, the melting temperature will be given roughly by the $2 \mathrm{D}$ Kosterlitz-Thouless melting temperature $T_{M}^{2 \mathrm{D}}$ which is only weakly field dependent for $H_{c 2} \gg B \gg H_{c 1}$. The phase diagram for such a quasitwo-dimensional system is shown schematically in Fig. 5.

By contrast, a less anisotropic material like $\mathrm{YBa}_{2} \mathrm{Cu}_{3} \mathrm{O}_{7}$ (Y-Ba-Cu-O), with $^{29} \gamma \simeq \frac{1}{5}$, will behave three dimensionally at accessible fields. At low temperatures the melting boundary will approach $H_{c 2}^{\mathrm{MF}}(T)$, but as $T$ increases it can drop considerably below this field. Indeed, in the $X Y$ critical regime $B_{M} \simeq(0.1) H_{X}$, where

$$
H_{X} \equiv \phi_{0} /\left(2 \pi \xi^{2}\right)
$$

is a crossover field equal to the mean field $H_{c 2}^{\mathrm{MF}}$ in the Ginzburg-Landau regime, but varying as $\left(T_{c}-T\right)^{2 v}$ in the $X Y$ critical regime, with $v \simeq \frac{2}{3}$. The phase diagram near $T_{c}$ on a $\log H$ versus $\log \left(T_{c}-T\right)$ scale is shown schematically in Fig. 6 for such a three-dimensional system, illustrating the crossovers between the various critical regimes.

In Sec. VI we consider the effects of random vortex pinning on the vortex-lattice phase. For simplicity, we concentrate on the effects of microscopic pinning (e.g., oxygen defects), although macroscopic defects may be important for obtaining large critical currents. As shown by Larkin and Ovchinikov, ${ }^{9}$ the vortex-lattice translational correlations are destroyed by pinning beyond a 


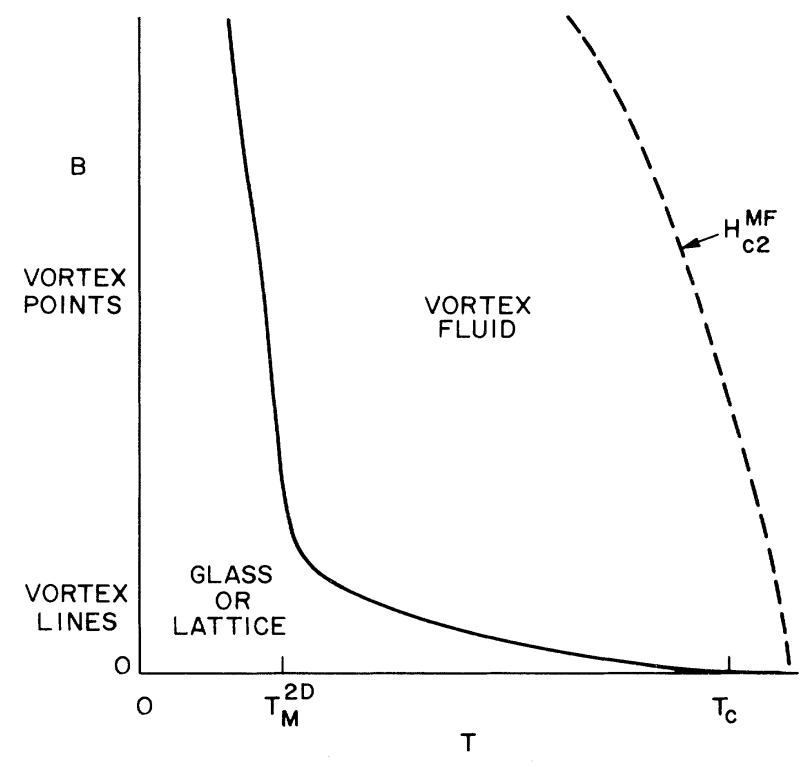

FIG. 5. Schematic phase diagram for a highly layered strongly type-II material like Bi-Sr-Ca-Cu-O. The crossover from the high-field two-dimensional regime of single vortices (vortex points) in each layer with weak interlayer interactions to the three-dimensional vortex-line regime at low fields occurs at a field scale given by the right-hand side of Eq. (5.6). At this crossover the transition temperature is of order that of an individual clean layer $T_{M}^{2 D}$, except perhaps for very strong disorder. The field is oriented normal to the layers.

length scale $L_{p}$. We estimate semiquantitatively the short-distance distortions in the lattice that are induced by the pinning in various regimes. We find that, at fixed temperature in both the two- and three-dimensional regimes, the lattice correlations measured in units of the lattice spacing $L_{p} / a_{v}$ decrease with increasing field for $B \gtrsim 2 H_{c 1}$. Thus, the direct observation of well-ordered flux lattices in Bitter decoration experiments at low fields, $B \lesssim 200 \mathrm{G}$, does not imply that these correlations persist at high fields where transport measurements are typically performed.

In Sec. VII we focus on the crossover from vortexlattice behavior to pinned behavior in the presence of weak pinning. If the melting transition in the absence of disorder is first order, as expected, then above this temperature there should be no large barriers, and the resistance will not be thermally activated. Just below this temperature the resistance will drop very rapidly and the $I-V$ characteristics become strongly nonlinear. In sum, for weak pinning the resistive transition must remain fairly sharp. The rather gradual (e.g., quasi-Arrhenius ${ }^{23}$ ) resistive transition that is generally seen in high- $T_{c}$ materials in large fields thus suggests that, for those samples, one may be in a strong-pinning regime.

The properties of the proposed vortex-glass phase are examined in Sec. VIII. We emphasize that, just like the Meissner phase and the Abrikosov vortex-lattice phase, the vortex-glass phase has off-diagonal long-range order in the pairing field $\psi$. However, the long-range order is in an essentially random pattern determined by the specific details of the pinning in the sample, just as occurs for the long-range magnetic order in a spin-glass phase.

The off-diagonal long-range order in the vortex-glass phase, although not directly observable, will manifest itself in transport properties. In this phase, in the presence of a current density $J$, there will be barriers for vortexloop (or vortex-bundle) excitations which grow for $J \rightarrow 0$ as $B(J) \sim J^{-\mu}$, with an exponent $\mu \leq 1$. Thermally activated vortex loops then lead to nonlinear dissipation which vanishes exponentially with current, as in (1.2). The current scale $J_{T}$ varies as $J_{T}^{\mu} \sim 1 / T$ at low temperature. As in the Meissner phase, there is a second current scale $J_{F}$ above which dissipation rapidly increases due to nonactivated vortex motion, i.e., vortex flow. At low temperatures a sharp critical current may be seen since $J_{F} \ll J_{T}$, but near the glass transition temperature $J_{F} \sim J_{T}$ and the $I-V$ characteristics should be rounded, as illustrated in Fig. 3.

In the vortex-glass phase a nonequilibrium current should decay with time (roughly) as ${ }^{30}$

$$
J(t) \approx J_{F}\left[1+(T / U) \ln \left(t / t_{0}\right)\right]^{-1 / \mu},
$$

with $U$ an effective barrier height such that $T / U \approx\left(J_{F} / J_{T}\right)^{\mu}$, and $t_{0} \sim 10^{-9} \mathrm{sec}$ is a microscopic "attempt" time. This form yields a $\partial J / \partial \ln t$ which is nonmonotonic in temperature at fixed time, in contrast to standard "flux-creep" theory.

The vortex-glass phase is a true superconductor with strictly zero linear resistivity, as is apparent from (1.2). Moreover, we argue that the frequency-dependent conductivity varies as $\sigma(\omega) \sim \rho_{s} /(-i \omega)$, so that the phase exhibits a nonzero superfluid density, just as in the Meissner phase. However, the equilibrium dc susceptibility $\partial M / \partial H$ is not equal to $-1 / 4 \pi$ in the vortex-glass phase, since additional dc magnetic fields will fully penetrate the sample. The penetration of an ac field becomes very inhomogeneous at low frequency, leading to various different measures of the penetration length, one of which diverges (logarithmically) for $\omega \rightarrow 0$, while another remains finite.

The continuous phase transition from vortex glass to vortex fluid is investigated in Sec. IX. In particular, we focus on the dc nonlinear current-voltage response and the ac linear conductivity near the transition, which are argued to have scaling forms similar to the zero-field transition, as in (1.4) and (1.5). The recent nonlinear $I-V$ data of Koch et al. and Gammel et al. ${ }^{22}$ have been fit quite successfully to the proposed scaling form, providing experimental evidence for the existence of the vortexglass phase. As also discussed in this section, the theoretical evidence for the existence of this phase arises from several related, but not fully realistic, models that have been studied both analytically ${ }^{11}$ and numerically. ${ }^{31}$

Section $\mathrm{X}$ contains a brief discussion of quasi-twodimensional effects in the presence of disorder which may be relevant for highly anisotropic materials like $\mathrm{Bi}-\mathrm{Sr}-\mathrm{Ca}-\mathrm{Cu}-\mathrm{O}$. In particular, the physics is conveniently 
described in terms of a $2 \mathrm{D}$ vortex-glass phase which exists only at $T=0$. As $T \rightarrow 0$, the $2 \mathrm{D}$ vortex-glass correlation length is expected to diverge, which should have observable consequences in the current-voltage characteristics.

Finally, in Sec. XI we present a discussion of some recent experiments, conclusions, and some open questions. Emphasis is placed on $\mathrm{Y}-\mathrm{Ba}-\mathrm{Cu}-\mathrm{O}$ and $\mathrm{Bi}-\mathrm{Sr}-\mathrm{Ca}-\mathrm{Cu}-\mathrm{O}$, which are the most studied of all the copper-oxide superconductors. These two materials exhibit rather different transport behavior in a magnetic field; the differences appear to be largely due to their different degrees of anisotropy.

\section{GINZBURG-LANDAU MODEL AND MEAN-FIELD THEORY}

In order to establish the important energy and length scales, we briefly review Ginzburg-Landau theory for a uniaxially anisotropic superconductor. The GinzburgLandau free-energy functional for a uniaxially anisotropic superconductor in applied magnetic field $\mathbf{H}$ is

$$
\begin{aligned}
F=\left.\int d^{3} r\left[\frac{\hbar^{2}}{2 m_{z}}|| \frac{\partial}{\partial z}-\frac{i 2 e}{\hbar c} A_{z}(\mathbf{r})\right] \psi(\mathbf{r})\right|^{2} \\
\left.+\frac{\hbar^{2}}{2 m_{\perp}}|| \nabla_{\perp}-\frac{i 2 e}{\hbar c} \mathbf{A}_{\perp}(\mathbf{r})\right]\left.\psi(\mathbf{r})\right|^{2} \\
+\alpha(T)|\psi(\mathbf{r})|^{2}+\frac{1}{2} \beta|\psi(\mathbf{r})|^{4} \\
\left.+\frac{1}{8 \pi}|\mathbf{H}-\boldsymbol{\nabla} \times \mathbf{A}(\mathbf{r})|^{2}\right]
\end{aligned}
$$

where $\boldsymbol{\nabla}_{\perp}$ and $\mathbf{A}_{\perp}$ represent the components of $\boldsymbol{\nabla}$ and $\mathbf{A}$ normal to the $z$ axis. The complex scalar orderparameter field $\psi(\mathbf{r})$ represents the wave functions of pairs of charge $2 e$. The final term couples the applied field to the magnetic induction $\mathbf{B}(\mathbf{r})=\boldsymbol{\nabla} \times \mathbf{A}(\mathbf{r})$, where $\mathbf{A}$ is the vector potential. For simplicity, we will generally assume the temperature dependence enters only through $\alpha(T)$ and that the applied field is parallel to the $z$ axis. We will always use $z$ subscripts to denote lengths in the $z$ direction, but, for notational simplicity, often drop the $\perp$ subscript for lengths in the planes normal to the $z$ axis. For stability, $\beta, m_{z}$, and $m_{\perp}$ are all positive. The smallscale quenched disorder we will consider is best modeled in (2.1) as a spatially random component of $\alpha: \delta \alpha(\mathbf{r}, T)$.

The mean-field minimization of (2.1) is well understood. ${ }^{1}$ Its phase diagram is Fig. 1. For $H=0$ and $\alpha(T)<0$, the system orders with $B=0$ and uniform $\psi$ in the gauge where $A=0$. In this Meissner phase the symmetry of $F$ under $\psi(\mathbf{r}) \rightarrow e^{i \phi} \psi(\mathbf{r})$ is spontaneously broken, resulting in off-diagonal long-range order. The meanfield magnitude of $\psi$ is given by $|\psi|^{2}=|\alpha| / \beta$. The meanfield coherence length for the decay of a perturbation of $|\psi|^{2}$ in the $x y$ plane is

$$
\xi_{\perp} \equiv \xi=\hbar /(2 m|\alpha|)^{1 / 2},
$$

where $m \equiv m_{1} ; \xi_{z}=\left(m / m_{z}\right)^{1 / 2} \xi$ is the mean-field coherence length along the $z$ direction. ${ }^{32}$ The mean-field mag- netic penetration length for a field screened by currents in the $x y$ plane (such as a field along the $z$ axis) is

$$
\lambda_{\perp} \equiv \lambda=\left(m c^{2} \beta / 16 \pi|\alpha| e^{2}\right)^{1 / 2} .
$$

For fields in the $z$ direction, the system is type II in mean-field theory when the ratio

$$
\kappa \equiv \lambda / \xi=(m c / 2 \hbar e)(\beta / 2 \pi)^{1 / 2}>2^{-1 / 2} .
$$

The penetration length for fields screened by currents along the $z$ axis is $\lambda_{z}=\left(m_{z} / m\right)^{1 / 2} \lambda$. For convenience we define an anisotropy parameter

$$
\gamma \equiv\left(\frac{m_{\perp}}{m_{z}}\right)^{1 / 2}
$$

Note that the anisotropies in $\xi$ and $\lambda$ are the reverse of each other: for $\gamma<1, \xi_{\perp}>\xi_{z}$ and $\lambda_{z}>\lambda_{1}$, so that one may define a $\kappa_{z} \equiv \lambda_{z} / \xi_{z}$ which differs from $\kappa$ by a factor of $\gamma^{-2}$.

In the limit of very weakly coupled layers, the interlayer coupling [first term in (2.1)] must be replaced by a gauge-invariant discrete derivative

$$
\left[\frac{\partial}{\partial z}-\frac{i 2 e}{\hbar c} A_{z}\right] \rightarrow \frac{1}{d} \Delta_{z}
$$

with $d$ the layer spacing and $\Delta_{z}$ the lattice derivative operator that yields the gauge-invariant difference between adjacent layers.

A small applied field $H<H_{c 1}$ is unable to penetrate the system so that the state is unchanged and $B$ remains zero. In this Meissner phase, the long-wavelength form of the free energy can be written in terms of the phase $\phi$ of the order parameter $[\psi=|\psi| \exp (i \phi)]$ as

$$
F=\int \frac{1}{2}\left(\nabla \phi-\frac{2 \pi \mathbf{A}}{\phi_{0}}\right) \overleftrightarrow{\rho}_{s}\left(\nabla \phi-\frac{2 \pi \mathbf{A}}{\phi_{0}}\right)+\frac{1}{8 \pi}(\nabla \times \mathbf{A})^{2},
$$

in terms of the normalized superfluid density tensor which is diagonal with components

$$
\left(\rho_{s}\right)_{i i}=\frac{\phi_{0}^{2}}{16 \pi^{3} \lambda_{i}^{2}}=\frac{T \Lambda_{T}}{\pi \lambda_{i}^{2}} .
$$

Above $H_{c 1}$ the field penetrates initially in isolated flux lines which contain vortices of the field $\psi$ and magnetic flux $\phi_{0}$. The mean-field free energy per unit length of such a flux line in the $z$ direction is $f=\varepsilon_{1}-H \phi_{0} / 4 \pi$, with

$$
\varepsilon_{1} \equiv \varepsilon_{1 z} \approx\left(\phi_{0} / 4 \pi \lambda\right)^{2} \ln \kappa
$$

for large $\kappa$. Thus, the magnetic field first penetrates at $H_{c 1}=4 \pi \varepsilon_{1} / \phi_{0}$. For $\kappa$ of order unity, $\varepsilon_{1}$ is roughly $\left(\phi_{0} / 4 \pi \lambda\right)^{2}$. (For simplicity, we generally analyze the 3D bulk behavior for a "needle crystal" oriented parallel to the applied field so demagnetization can be ignored. For other sample geometries $H_{c 1}$ is altered.)

For $H_{c 1}<H<H_{c 2}=\phi_{0} / 2 \pi \xi^{2}$ the mean-field minimum of (2.1) is an Abrikosov vortex lattice. The vortices are all parallel to the applied field and frozen into a triangu- 
lar array. Thus, in this vortex-lattice phase the symmetry under continuous translations in the $x y$ plane is also spontaneously broken. The applied field $H$ frustrates the system more and more as it is increased, and the order parameters are driven to zero at $H_{c 2}$.

In the absence of bulk vortex pinning, the vortexlattice phase is not a superconductor for currents running normal to the field, but instead has a nonzero Ohmic resistivity due to "flux flow." 8

The characteristic frequency scale in the vortex-lattice phase is determined by the forces between the vortex lines and their mobility; the latter also determines the flux-flow resistivity. A simple mean-field estimate of the relaxational frequency of a (overdamped) zone-boundary (ZB) flux-lattice "phonon" for $H_{c 1}<H<<H_{c 2}$ yields

$$
\begin{aligned}
& \omega_{\mathrm{ZB}} \sim \frac{\rho_{F} c^{2}}{16 \pi^{2} \lambda^{2}(T)} \\
& \simeq \frac{H}{H_{c 2}(T=0)} \frac{\rho_{N} c^{2}}{16 \pi^{2} \lambda^{2}(T=0)} \\
& \simeq 6 \times 10^{10} \mathrm{sec}^{-1} \frac{H}{H_{c 2}(0)}\left[\frac{\rho_{N}}{\mu \Omega \mathrm{cm}}\right)\left[\frac{1000 \AA}{\lambda(0)}\right]^{2}
\end{aligned}
$$

in terms of the in-plane flux-flow resistivity $\rho_{F}(H, T)$ which, in the second equality, we assume ${ }^{8}$ is roughly given by $\rho_{N} H / H_{c 2}(T)$ with $\rho_{N}$ the normal-state resistivity in the mean-field regime just above the zero-field transition temperature $T_{c}$. Near $H_{c 1}$ or $H_{c 2}$ this frequency will be smaller than given by (2.6).

Mean-field theory exhibits two ordered phases, the Meissner phase and the vortex-lattice phase, and three distinct transitions which will be, to varying degrees, altered by thermal fluctuations and quenched disorder. These are (i) the transition at $H=0$ as $\alpha(T)$ passes through zero, (ii) the Meissner-to-vortex-lattice transition at $H_{c 1}$, and (iii) the vortex-lattice-to-normal transition at $H_{c 2}$.

\section{THE MEISSNER PHASE}

We first examine the simplest phase. In the Meissner phase the system superconducts, with no dissipation occurring which is linear in the current density $J$. In a mean-field analysis there is no dissipation at all up to a critical current density where the system goes normal. Thermal fluctuations alter this, however, yielding dissipation due to thermally activated nucleation and subsequent growth of vortex loops at any nonzero current density. ${ }^{25}$ Let us examine these processes for a bulk superconductor, neglecting the processes that occur near the surfaces that are actually more important in any finitesized sample at low enough currents. At low current densities, the magnitude of the order parameter is not significantly altered and the free energy of a circular vortex loop lying in a plane normal to the current, of radius $R>\lambda$ and having the appropriate sign of the circulation, is

$$
F_{\text {loop }} \approx 2 \pi R \varepsilon_{1}-J \frac{\phi_{0}}{c} \pi R^{2}
$$

where, again, $\varepsilon_{1}$ is the free energy per unit length of the vortex line and we first consider the isotropic case $(\gamma=1)$ for simplicity. (Note we are assuming the current density is uniform, which is not strictly true for very low current densities where the current is concentrated near the other moving vortices.) The critical radius at which $F_{\text {loop }}$ has its maximum is $R_{c} \approx c \varepsilon_{1} / J \phi_{0}$ and the resulting freeenergy barrier is $\pi c \varepsilon_{1}^{2} / J \phi_{0}$. Thus, the activated dissipation rate or resistivity is

$$
\rho \equiv \frac{E}{J} \sim e^{-J_{T} / J},
$$

with the characteristic current scale set by these thermal fluctuations given by

$$
J_{T} \approx \pi c \varepsilon_{1}^{2} / \phi_{0} T .
$$

For $R$ in the range $\xi<<R \lesssim \lambda,(3.1)$ is not precisely correct since $\varepsilon_{1}$ varies as $\ln (R / \xi)$ in this range; however, this does not alter the exponential dependence of $\rho$ on $J$ in (3.2); it just changes the (unknown) prefactor.

When the current is large enough that the critical radius becomes of order $\xi$, the above picture breaks down: this occurs for $J$ near a current density $J_{F}$ which is when the onset of strong nonlinear (and nonactivated) phase slip occurs. This will be of order the mean-field (depairing) critical current: $J_{F} \sim J_{c}^{\mathrm{MF}} \sim\left(c \varepsilon_{1}\right) / \phi_{0} \xi$. The loop nucleation barrier for currents just below this mean-field critical current is of order $\varepsilon_{1} \xi$. Thus, the resistivity at currents near but below the mean-field critical current is of order $\exp \left(-\varepsilon_{1} \xi / T\right)$ times the normal resistivity. In conventional materials this is very small except very close to $T_{c}$. This is a reflection of the occurrence of two very different current scales: $J_{T}$ for the thermally activated phase slip and $J_{F}$ for deterministic (nonactivated) creation of vortex loops. In the limit $J_{T} \gg J_{F}$, there is a sharp change in behavior and an apparent critical current of order $J_{F}$ as illustrated in Fig. 3(a). It is such a wide separation of current scales which hides the effects of thermal fluctuations in conventional bulk superconductors. As $T_{c}$ is approached, however, $J_{T}$ decreases more rapidly than $J_{F}$. When they become comparable, thermal fluctuations play an important role and $I-V$ curves become rounded and much smoother as in Fig. 3(b). We will see below that this criterion $\left(J_{T}\right.$ of order $\left.J_{F}\right)$ is essentially the same as the Ginzburg criterion.

In an anisotropic material, the critical vortex loop is elliptical rather than circular. The characteristic current $J_{T} \approx \pi c\left(\varepsilon_{1 \perp} \varepsilon_{1 z}\right) / \phi_{0} T$ for in-plane current where

$$
\varepsilon_{1 \perp} \approx \gamma \varepsilon_{1 z} \propto \frac{1}{\lambda_{\perp} \lambda_{z}}
$$

is the free energy per unit length of a vortex line parallel to the planes. The mean-field critical current is when the critical loop has axes $\xi_{z}$ and $\xi_{\perp}$. The critical region is then when $\varepsilon_{1 \perp} \xi_{\perp} \sim \varepsilon_{1 z} \xi_{z} \sim T$ which, due to the inverse dependence of $\xi$ and $\lambda$ on anisotropy, will happen con- 
currently.

In the presence of quenched disorder insufficient to destroy superconductivity, the nonlinear current-voltage behavior in the Meissner phase will not be significantly changed. However, the existence of random regions in which vortices have lower free energy will cause longtime tails in equilibrium correlation functions due to long-lived thermally excited vortex loops. ${ }^{33}$ These will give rise to singular low-frequency corrections to the mean-field ac linear conductivity, probably of the form of nonuniversal powers of $\omega$. We also note in passing that, even at zero temperature, there will be dissipation at any nonzero $J$ from quantum tunneling through the above discussed barriers.

\section{THE NORMAL-TO-SUPERCONDUCTOR TRANSITION AT $H=0$}

We now consider the critical behavior in zero field. A superconductor is type II when the Cooper pair field $\psi$ is "softer" than the vector potential field A. Thus, the $\psi$ field has stronger thermal fluctuations and it is these fluctuations that first start to alter the critical behavior as $T_{c}$ is approached. The point at which the system crosses over from mean-field to fluctuation-dominated critical behavior has been called the "Ginzburg criterion." For $T<T_{c}$, one can use the simple criterion of when the ordering free energy in a coherence volume is equal to the temperature: $\alpha^{2} \xi^{2} \xi_{z} / 2 \beta \simeq T$. This translates to

$$
\lambda \simeq \frac{\gamma \phi_{0}^{2}}{16 c_{s} \pi^{3} T \kappa}=\frac{\gamma \Lambda_{T}}{c_{s} \pi \kappa},
$$

with the constant $c_{s}=4$. We will see later that there are other sensible criteria for this crossover which yield a $c_{s}$ smaller by an order of magnitude and more, and thus a crossover much closer to $T_{c}$. The criterion (4.1) is (up to the constant) the same condition as $J_{T} \simeq J_{F}$ discussed in the previous section. In the high- $T_{c}$ cuprate superconductors $T, \kappa$, and $\gamma^{-1}$ are all larger than in conventional superconductors. This makes the regime of fluctuationdominated critical behavior potentially accessible.

In mean-field theory, the resistivity has a discontinuity at $T_{c}$. This jump is found experimentally to be rounded on the scale of order a tenth of a degree $\mathrm{K}$ in, for example, $\mathrm{YBa}_{2} \mathrm{Cu}_{3} \mathrm{O}_{7}(\mathrm{Y}-\mathrm{Ba}-\mathrm{Cu}-\mathrm{O})$. It is possible that some of this rounding may actually be due to inhomogeneity of the samples, but it may also indicate the fluctuationdominated critical regime. The mean-field discontinuity of the specific heat, on the other hand, should be replaced in the critical regime by a nearly logarithmic divergence, since the specific-heat exponent is $\alpha \simeq-0.016$ for $X Y$ critical behavior ${ }^{34}$ (see below). Thus, a cusped peak in the specific heat is indicative of fluctuation effects, while rounding of that peak is indicative of sample inhomogeneities. Note that the above discussion assumes three dimensionality; that is, $\xi_{z}>d$ at the Ginzburg temperature with $d$ the layer spacing. For the two-dimensional regime $\left(\xi_{z}<d\right)$, the Ginzburg criterion is $\alpha^{2} \xi^{2} d / 2 \beta \simeq T$. However, in this limit, the behavior can better be described in terms of weakly coupled layers with almost in- dependent Kosterlitz-Thouless transitions in the layers; other criteria for the fluctuation regime are then more useful. ${ }^{35}$

We now examine the behavior in the fluctuationdominated critical regime. This serves two purposes, the first being that this regime may actually be accessible at $H=0$ if sufficiently homogeneous samples are obtained, and the second is that the scaling here is fairly similar to that expected at a finite-temperature vortexfluid-to-vortex-glass transition which will be examined in Sec. IX.

The effective dimensionless charge of a mean-field superconductor which determines the strength of the screening is

$$
\widetilde{e}=1 / \kappa=e \frac{2 \hbar}{m c}\left(\frac{2 \pi}{\beta}\right)^{1 / 2} .
$$

In a strongly type-II system, this normalized charge is small and the screening is weak, as reflected by $\lambda \gg \xi$. The crossover upon approaching $T_{c}$ is thus initially to the critical regime of a weakly charged superfluid where the fluctuations in $\psi$ are essentially those of an uncharged superfluid or $X Y$ model. In this regime, $\xi \sim\left|T-T_{c}\right|^{-v}$ with $v \simeq \frac{2}{3}$ in three dimensions $(D=3)$. For $T<T_{c}$, the superfluid density scales as ${ }^{36} \rho_{s} \sim \xi^{2-D}$. Thus, the penetration length scales as $\lambda \sim \rho_{s}^{-1 / 2} \sim \xi^{(\dot{D}-2) / 2}$ in this $X Y$ critical regime. This results in a renormalization of $\kappa$ as $\kappa \sim \xi^{(D-4) / 2}$. For $D=3$, the condition (4.1) will thus be satisfied throughout this regime with $c_{s}$ a universal constant.

In order to estimate $c_{s}$ we need to specify our convention for defining $\xi$ for $T<T_{c}$. For $T>T_{c}$, we define $\xi$ as the exponential decay length for the correlations in the field $\psi$. For $T<T_{c}$, the decay is nowhere exponential so we simply define $\xi$ so it is symmetric about $T_{c}$, namely, $\xi(\varepsilon) \equiv \xi(-\varepsilon)$ with

$$
\varepsilon \equiv \frac{T-T_{c}}{T_{c}} \text {. }
$$

In the $X Y$ critical regime for $T<T_{c}$ we have

$$
\xi_{\perp} \approx \xi_{10}|\varepsilon|^{-v}
$$

and

$$
\lambda_{1} \approx \tilde{\lambda}_{10}|\varepsilon|^{-v / 2}
$$

with

$$
\frac{\tilde{\lambda}_{\perp 0}^{2}}{\tilde{\xi}_{\perp 0}}=\frac{\gamma \Lambda_{T_{c}}}{\pi c_{s}} .
$$

The universal constant here is estimated ${ }^{37}$ to be $c_{s} \simeq 0.4$. In the Ginzburg-Landau (mean-field) regime, one has instead

$$
\begin{aligned}
& \xi_{\perp} \approx \xi_{\perp 0}|\varepsilon|^{-1 / 2}, \\
& \lambda_{\perp} \approx \lambda_{\perp 0}|\varepsilon|^{-1 / 2} .
\end{aligned}
$$

The crossover point $\varepsilon_{x}$ can then be defined as the point where the Ginzburg-Landau expression for $\lambda^{2} / \xi$ coin- 
cides with (4.6), yielding

$$
\varepsilon_{x}=\left(\frac{\lambda_{\perp 0}^{2}}{\xi_{\perp 0}} \frac{\pi c_{s}}{\gamma \Lambda_{T_{c}}}\right)^{2},
$$

which is of order $10^{-2}$ to $10^{-1}$ for $\mathrm{Y}-\mathrm{Ba}-\mathrm{Cu}-\mathrm{O}$ if we estimate $\lambda_{10} \simeq 800 \AA, 30 \lesssim \kappa \lesssim 120$, and $\gamma \simeq 0.2$, but may be larger for $\mathrm{Bi}-\mathrm{Sr}-\mathrm{Ca}-\mathrm{Cu}-\mathrm{O}$ due to the smaller $\gamma$. A twodimensional criterion is likely to be more appropriate for $\mathrm{Bi}-\mathrm{Sr}-\mathrm{Ca}-\mathrm{Cu}-\mathrm{O}$ however.

We note that there is considerable ambiguity in defining the width of the critical region. For example, the standard Ginzburg criterion, that the leading fluctuation correction to the specific heat as $T$ approaches $T_{c}$ from above be equal to the mean-field specific-heat discontinuity, gives an expression similar to Eq. (4.8) except with a factor of $\frac{1}{4}$ instead of $\pi c_{s}$ yielding an $\varepsilon_{x} 25$ times smaller than (4.8). However, this standard Ginzburg criterion appears to be an underestimate of the width of the critical regime, because the higher-order fluctuation corrections are significantly larger than the leading terms at this point. Thus, the crossover between the mean-field and fluctuation critical regimes appears to occur when the singular fluctuation corrections to the specific heat are still significantly smaller than the meanfield specific-heat jump. The situation for the conductivity appears to be similar, with the crossover occurring where the fluctuation conductivity ${ }^{2}$ is a fairly small correction. Detailed calculations of the meanfield-to $-X Y$ crossover are needed for any real comparison with experiments.

In the $X Y$ critical regime, since $\kappa \sim \xi^{(D-4) / 2}$, the charge is relevant ${ }^{26}$ for $D<4$, and $\kappa$ decreases as one approaches $T_{c}$. When $\kappa$ becomes of order unity the system crosses over into another critical regime, that of a charged superfluid. ${ }^{27}$ For an isotropic material we expect $T \xi \kappa^{2} \sim\left(\phi_{0} / 4 \pi\right)^{2}$ in the intermediate critical regime, so the final crossover occurs when $T \xi \simeq 10^{8} \mathrm{~K} \AA$, which is a very large coherence length $(\simeq 0.1 \mathrm{~mm})$, even for the high- $T_{c}$ materials.

In anisotropic materials, however, $\kappa=\kappa_{\perp}$ (which we assume is $\left\langle\kappa_{z}\right.$ ) will become of order unity when

$$
\xi_{\perp} \sim \lambda_{\perp} \sim \gamma \Lambda_{T}
$$

and at this point the magnetic field fluctuations will start to become important; however, the system is still anisotropic. What the eventual critical behavior will be is unclear in this anisotropic case and we will primarily restrict consideration in this paper to the intermediate $X Y$ critical regime. Note, however, that with small enough $\gamma$ (high anisotropy), the magnetic-field fluctuation critical regime may become accessible experimentally in extremely good samples. The various critical regimes are shown on log scales in Fig. 6.

What dynamic universality class is the intermediate $(X Y)$ critical regime? In the absence of Coulomb interactions, one would expect model- $E$ dynamics to apply with the conserved charge-density conjugate to the phase fluctuations. ${ }^{38}$ However, the plasma fluctuations (with a plasmon gap) probably effectively negate this effect and the appropriate universality class appears to be that with the number of Cooper pairs not conserved. This suggests that a fully relaxational dynamics (model $A$ ) should be appropriate. ${ }^{39}$ However, most of the expressions we obtain should be generally valid but with the value of the dynamic scaling exponent $z$ and the detailed forms of the scaling functions determined by the appropriate dynamic universality class.

Since the specific-heat exponent $\alpha$ is very close to zero for the 3D $X Y$ model universality class that applies at this $H=0$ critical point, quenched disorder is effectively a marginally irrelevant operator. Thus, weak disorder should not change the critical behavior significantly, although strong disorder will yield large corrections to scaling from the slow transients in the renormalizationgroup flows near the critical fixed point. ${ }^{40}$ We now examine the critical scaling of those quantities that are peculiar to the clean, weakly charged, rather than strictly unchanged, superfluid.

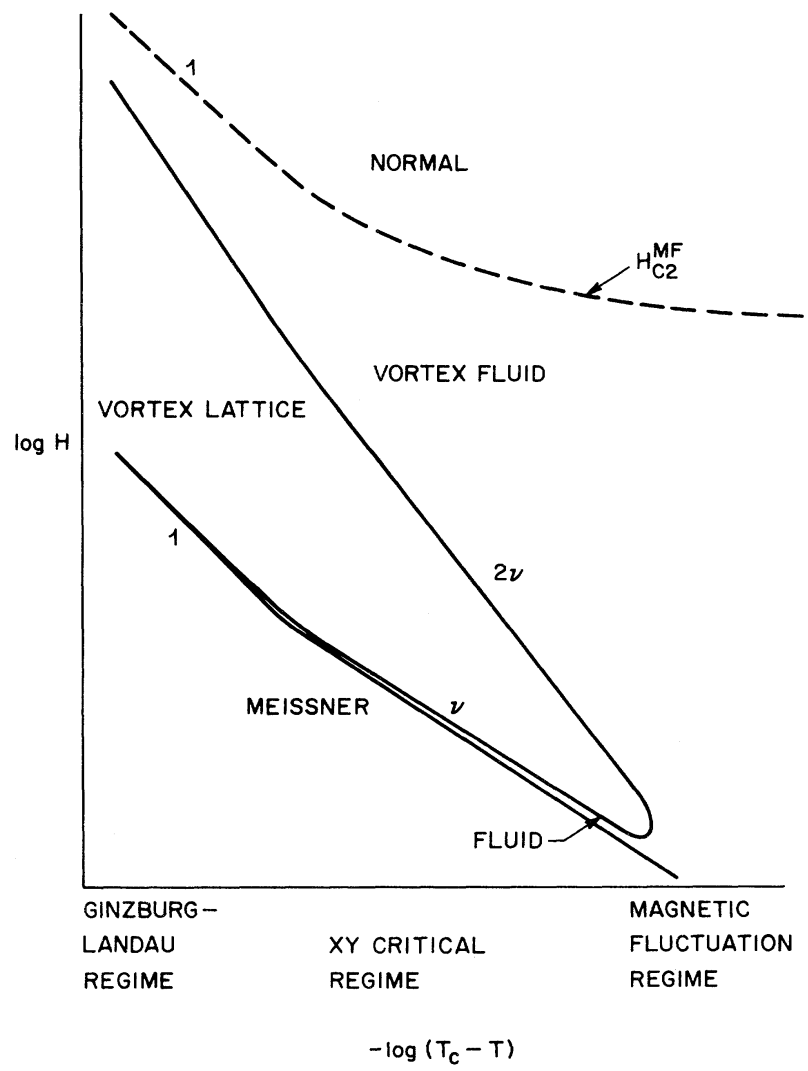

FIG. 6. Schematic phase diagram in the region near $T_{c}$ for a system with no pinning showing the critical regimes and crossovers on log scales. The system is assumed to be behaving three dimensionally throughout. The numbers $(1, v, 2 v)$ next to the phase boundaries and crossover line denote their slopes on this $\log -\log$ plot. The crossover from the $X Y$ critical regime to the magnetic fluctuation critical regime occurs near where the vortex-lattice phase terminates. 


\section{A. Fluctuation diamagnetism}

The appropriate scaling of the magnetic field in the intermediate critical regime is in terms of the number of flux quanta per coherence area. Thus, we expect the singular part of the free energy per unit volume to scale as

$$
f_{s} \approx T \xi^{-D \widetilde{F}}\left(H \xi^{2} / \phi_{0}\right),
$$

where $\widetilde{\mathscr{F}}$ is a scaling function. The resulting expression for the divergent part of the diamagnetic susceptibility is then

$$
\chi_{s}^{\prime}=\left.\frac{\partial^{2} f_{s}}{\partial H^{2}}\right|_{H=0} \sim-\frac{T}{\phi_{0}^{2}} \xi^{4-D} \sim \frac{-\xi}{\Lambda_{T}}
$$

(the final expression is for 3D), which applies both in the mean-field regime ${ }^{2}$ and the intermediate critical regime. [Note that the dependence of $\xi$ on $\left(T-T_{c}\right)$ will change at the Ginzburg crossover, as will the proportionality constants not shown in (4.10).] For $\gamma \simeq 1$ (isotropic), we see that $\chi_{s}^{\prime}$ is of order unity (full diamagnetism) only at the final crossover to the magnetic-field fluctuation regime where $T \xi \sim \phi_{0}^{2}$. For anisotropic layered materials, $\chi_{z z}$ will become of order unity at the point when $\kappa_{\perp} \sim 1$, i.e., the same criterion for the crossover to the magnetic fluctuation critical regime as given in (4.9).

\section{B. Frequency-dependent conductivity}

For $T<T_{c}$ and low frequency, the complex conductivity has the form

$$
\sigma(\omega) \approx \rho_{s} /(-i \omega+\varepsilon),
$$

where $\varepsilon \rightarrow 0+$, the real part thus having a $\delta$ function at zero frequency. Noting that, in the critical region, ${ }^{36}$ $\rho_{s} \sim \xi^{2-D}$ and that the characteristic relaxation time scales as $\tau \sim \xi^{z}$, this indicates that the low-frequency linear conductivity should scale as

$$
\sigma(\omega) \approx \xi^{z+2-D} \mathcal{\rho}_{ \pm}\left(\omega \xi^{z}\right),
$$

where the complex scaling functions $\mathcal{S}_{+}$apply above $(+)$ and below $(-) T_{c}$. The scaling functions behave as $\mathcal{S}_{+}(x) \rightarrow$ real constant and $\mathcal{f}_{-}(x) \sim 1 /(-i x)$ for $x \rightarrow 0$, reflecting the low-frequency behavior above and below $T_{c}$, while

$$
\mathcal{S}_{+}(x) \approx \mathcal{S}_{-}(x) \approx \widetilde{c} x^{[(D-2) / z-1]}
$$

with $\widetilde{c}$ a complex constant for $x \rightarrow \infty$, representing $T \rightarrow T_{c}$. Thus, at $T_{c}$, both the real and imaginary parts of $\sigma$ diverge for $\omega \rightarrow 0$ as

$$
\sigma(\omega) \sim \omega^{-(z+2-D) / z} .
$$

Note that, for $T \rightarrow T_{c}^{+}$, the dc conductivity $\sigma(0)$ diverges as $\xi^{z+2-D}$. This yields the correct results for the upper $(D=4)$ and lower ${ }^{19}(D=2)$ critical dimension since, for both cases, relaxational dynamics gives $z=2$.

A useful dimensionless quantity that might be used to locate the transition is the phase angle, $\phi_{\sigma}$ defined by $\sigma=|\sigma| e^{i \phi_{\sigma}}$. From (4.11) this should scale as

$$
\phi_{\sigma}(T, \omega) \approx P_{\sigma}\left(\left(T-T_{c}\right) \omega^{-1 / z v}\right),
$$

where $P_{\sigma}(x) \rightarrow 0$ for $x \rightarrow \infty$ and $P_{\sigma}(x) \rightarrow \pi / 2$ for $x \rightarrow-\infty$. At $T_{c}$ the low-frequency phase angle is a constant, $P_{\sigma}(0)$. Above the upper critical dimension $(D=4)$, $P_{\sigma}(0)=0$ because the system behaves like a normal metal even at $T_{c}$. At the lower critical dimension $(D=2)$, on the other hand, $P_{\sigma}(0)=\pi / 2$ because $\rho_{s}>0$ at $T_{c}$ for a Kosterlitz-Thouless transition. Thus, for $D=3$, we might guess that $P_{\sigma}(0)$ is well away from both 0 and $\pi / 2$ for this zero-field transition. Causality of $\sigma(\omega)$ combined with (4.12) indicates ${ }^{41}$ that, for $0<(z+2-D) / z<1$,

$$
P_{\sigma}(0)=\frac{\pi}{2} \frac{z+2-D}{z} \text {. }
$$

\section{Nonlinear conductivity}

In order to investigate the scaling of the nonlinear conductivity in the zero-field (intermediate) critical region, it is instructive to go back to Sec. III and examine the two characteristic current densities which appear in the Meissner phase: $J_{T}$ and $J_{F}$. In the intermediate critical region, both of these characteristic in-plane current densities are of order

$$
J_{X \perp} \sim \frac{c T_{c}}{\phi_{0}} \frac{1}{\xi_{\perp} \xi_{z}} .
$$

Thus, the characteristic current density is directly related to the critical correlation lengths. An argument, analogous to that of Josephson (see also, Sec. IX below) for the scaling of the superfluid density ${ }^{36}$ shows that this is true in general, and does not depend on the interpretation in terms of vortex loops: The characteristic current density will be that which is sufficiently large to appreciably change the statistics of the phase and vortex-line fluctuations. In a correlation volume of an isotropic system, the typical variation in the phase will be $\sim 2 \pi$ so that $|\nabla \phi| \sim 1 / \xi$ (where $\left.\psi \equiv|\psi| e^{i \phi}\right)$. The coupling of an external current to a phase gradient is via

$$
\delta F_{J}=-\int \frac{\phi_{0}}{c} \mathbf{J} \cdot \nabla \phi
$$

since the (mass) supercurrent is conjugate to the superfluid velocity. In a correlation volume $\xi^{D}, \delta F_{J}$ will be of order $T$, and thus of similar magnitude to the spontaneous fluctuations when $J \sim(c T) / \phi_{0} \xi^{D-1}$. For an anisotropic 3D system, the in-plane characteristic current will, by the analogous argument, be given by Eq. (4.15). In the $z$ direction the characteristic current density is

$$
J_{X z} \sim \frac{c T}{\phi_{0}} \frac{1}{\xi_{\perp}^{2}},
$$

which is smaller than $J_{X \perp}$ for $\gamma<1$.

For an isotropic system, we thus expect the dissipative electric field with a dc current density $J$ to scale as

$$
E \approx J \xi^{D-2-z} \mathscr{E}_{ \pm}\left(J \phi_{0} \xi^{D-1} / c T\right)
$$

for general $D$. The scaling functions should behave as 
$\mathscr{E}_{+}(x) \rightarrow$ constant as $x \rightarrow 0$ above $T_{c}$, corresponding to finite resistivity; and (for $D=3$ ) $\mathscr{E}_{-}(x) \sim e^{-a / x}$ for $x \rightarrow 0$ below $T_{c}$ due to vortex-loop nucleation as in (3.2), with an $a$ constant. At $T_{c}$,

$$
\mathscr{E}_{+}(x) \approx \mathscr{E}_{-}(x) \sim x^{(z+2-D) /(D-1)}
$$

for $x \rightarrow \infty$. This results in

$$
E \sim J^{(z+1) /(D-1)}
$$

at $T_{c}$, which, again with $z=2$, agrees with the expected Ohmic behavior at $T_{c}$ in $D=4$ and the ${ }^{19} E \sim J^{3}$ behavior at $T_{c}$ in $D=2$. Thus, for $D=3$ we expect the conductivity very near $T_{c}$ to obey (4.19) for currents much bigger than $J_{X}$ but not too large and to cross over to Ohmic behavior at low currents for $T>T_{c}$ and to exponentially small dissipation at low currents for $T<T_{c}$. For an anisotropic $3 \mathrm{D}$ system with current $J_{\perp}$ in the planes, $J \xi^{D-1}$ in (4.18) is replaced by $J_{\perp} \xi_{\perp} \xi_{z}$.

These scaling forms for the linear ac and nonlinear dc conductivity should apply generally, although with possibly different exponents and scaling functions, for any second-order normal-to-superconductor transition when the effects of screening can be ignored. Thus, they should also apply for the vortex-glass-to-vortex-fluid transition in a magnetic field with substitution of the appropriate vortex-glass correlation length, critical exponents, and scaling functions, as is discussed below in Sec. IX.

\section{VORTEX-LATTICE PHASE AND MELTING TRANSITIONS}

We now turn to the vortex-lattice phase and consider the effects of thermal fluctuations. The fluctuations give rise to overdamped phononlike excitations of the vortex lattice. When these fluctuations get sufficiently large, the system is expected to melt into a vortex liquid, as discussed by several authors ${ }^{6,7,15-18}$ and outlined below. There will also be fluctuations in the form of dislocation loops somewhat analogous to the vortex loops discussed in the previous section. Dislocation loops are necessary for the melting of a vortex lattice into a fluid phase to occur. ${ }^{42}$ These dislocation loops will also give rise to the activated relaxation of shear stresses in the vortex-lattice phase by nucleation and growth processes, in a similar fashion as activated vortex loops relax the current in the Meissner phase. Such processes could occur under conditions of nonuniform current density and may be important in determining the behavior in the presence of large length scale disorder (e.g., widely spaced pinning states).

In the absence of pinning of vortex lines, a vortex lattice will move with a nonzero linear mobility in response to any uniform applied current which has a component normal to the vortex lines. This motion produces slip in the phase of the field $\psi$, dissipation, and, hence, a nonzero electrical resistance. Thus, this phase is not really superconducting. (The question of what happens in the presence of thermal fluctuations when a very small current is precisely parallel to the vortex lines appears not to have been addressed.) In any real material, however, there will be defects which give rise to energetically preferred posi- tions for the vortex lines and thus inhibit their motion.

The long-range crystalline order of the vortices in the vortex-lattice phase is unstable against the introduction of quenched disorder or random pinning ${ }^{9}$ as discussed in more detail in the next section. Thus, the correlation length for the crystalline positional order of the vortices will be finite when there is quenched disorder present in the system. The vortex-lattice phase may then be replaced by a vortex-glass phase ${ }^{10,11}$ which still has longrange spin-glasslike order in the pairing field $\psi$. Some properties of this vortex-glass phase are discussed in Sec. VIII below. The possible phase transition between the vortex-glass phase and a vortex fluid will be discussed in Sec. IX. An alternative scenario is that the correlation length for the spin-glasslike order could remain finite all the way to zero temperature (diverging at $T=0$, see below), and the system remain a fluid, albeit a glassy one, as in the traditional Anderson-Kim ${ }^{3,4}$ theory of flux creep.

It is important to distinguish two main types of pinning: small-scale disorder from impurities, vacancies, etc., and large-scale disorder from well-spaced grain or twin boundaries, cracks, macroscopic inhomogeneities, etc. The relevant length scale with which to compare the pinning correlations is the spacing between vortices: thus, a high density of randomly placed twin or grain boundaries may result in effectively small-scale disorder if the density of such defects is homogeneous on larger scales. Small-scale disorder, which we discuss in detail in the following section, destroys the long-range positional correlations of the vortex lattice and, as we will argue, may give rise to a superconducting vortex-glass phase. Large-scale disorder, on the other hand, can leave the lattice structure intact out to long distances but can nevertheless drastically hinder macroscopic flux flow. In this case, which we will not discuss further, the melting of the vortex lattice in regions between defects may have large consequences for the resistance, since a vortex fluid will not be impeded from flowing nearly as much as a lattice would. In principle, with controlled, widely spaced, strong-pinning regions with very clean material in between, it should be possible to directly measure properties of a vortex lattice such as elastic moduli and also plastic flow under large current-induced stresses.

Before considering the effects of pinning in more detail (in the next section), we first discuss the transition, in a clean system, from the vortex lattice to a vortex-fluid phase as the thermal fluctuations increase. We do this for two reasons, first to point out some aspects of this transition that appear to have been missed in recent studies, and secondly because, in the presence of weak disorder, we expect the resistance to show marked changes near the pure system flux-lattice melting boundaries, as is discussed in Sec. VII below. For the remainder of this section we consider the system without pinning.

In mean-field theory (Fig. 1), there are two different types of second-order phase boundaries at which the vortex-lattice disappears: as the field is decreased at $H_{c 1}$ with a transition directly to the Meissner phase and as the field is increased through $H_{c 2}$ with a transition to the normal phase. We examine these in this order. 


\section{A. Melting near $\boldsymbol{H}_{c 1}$}

In mean-field theory the transition at $H_{c 1}$ is continu. ous, with the lattice constant of the Abrikosov vortex lattice going to infinity for $H \rightarrow H_{c 1}^{+}$. However, the interaction between parallel vortices falls off as $\exp (-r / \lambda)$ for vortex separation $r \gg \lambda$ and the lattice will thus be highly unstable to thermal fluctuations very close to $H_{c 1}$. Thus, in a superconductor free from random pinning, a region of vortex-fluid phase introduces at $H_{c 1}$ for all $T>0$, as has been recently emphasized ${ }^{6,7,15}$ and is illustrated in Fig. 4. (Note, this region of "reentrant" vortex fluid will be very unstable to pinning, as discussed below.) In order to estimate the width of this fluid phase we need to know the stiffness of a single vortex line to tilting. If the free energy per unit length projected on the $z$ axis of a vortex line running at angle $\theta$ to the $z$ axis is

$$
f=\varepsilon_{1}+\frac{1}{2} \widetilde{\varepsilon}_{1} \theta^{2}+O\left(\theta^{4}\right),
$$

then the width in applied field $\Delta H_{f}$ of the fluid phase is

$$
\frac{\Delta H_{f}}{H_{c 1}} \sim \frac{T^{2}}{\lambda^{2} \varepsilon_{1} \widetilde{\varepsilon}_{1}},
$$

as obtained by Nelson and Seung. ${ }^{7}$ For an isotropic superconductor, $\widetilde{\varepsilon}_{1}=\varepsilon_{1}$ and thus $\Delta H_{f} / H_{c 1} \sim \lambda^{2} / \Lambda_{T}^{2}$ (up to logarithmic factors). Even in the $X Y$ model fluctuation critical regime where $\varepsilon_{1} \sim T / \xi, \Delta H_{f} / H_{c 1}$ is only of order $1 / \kappa^{2}$. Thus, the width of this fluid phase in a strongly type-II isotropic system is quite small in units of $H_{c 1}$ except extremely near $T_{c}$ where $\kappa$ becomes temperature dependent. Now one might have thought ${ }^{6}$ that the tilt stiffness $\widetilde{\varepsilon}_{1}$ can be made arbitrarily small relative to $\varepsilon_{1}$ by taking a highly anisotropic system with small $\gamma$, thereby increasing $\Delta H_{f}$. However, the electromagnetic coupling [the $|\mathbf{H}-\boldsymbol{\nabla} \times \mathbf{A}|^{2}$ term in the free energy (2.1)] is intrinsically isotropic, so this is not, in fact, correct.

To see this, it is instructive to consider a highly anisotropic, quasi-two-dimensional layered superconductor, like Bi-Sr-Ca-Cu-O. Near $H_{c 1}$ the intruding magnetic field is confined to the vicinity of the vortex. The vortex line free energy in mean-field theory is $\varepsilon_{1} \approx\left(\phi_{0} / 4 \pi \lambda\right)^{2} \ln \kappa$ for large $\kappa$ of which $\varepsilon_{1 M} \sim\left(\phi_{0} / 4 \pi \lambda\right)^{2}$ is magnetic energy, the remainder being due to the "strain" in the $\psi$ field in each plane which thus dominates for an isotropic system. On the other hand, if the Josephson coupling between planes is very weak (small $\gamma$ ), then, when we tilt a vortex, the $\psi$ fields in each plane can remain almost unchanged relative to the position where the point vortex passes through the plane. In the limit of small $\gamma$, the increase in free energy per plane is just due to the tilting of the magnetic field and the coupling of the magnetic field of a vortex in one plane to the phase gradients (thus currents) in other planes. This magnetic energy per unit length yields $\widetilde{\varepsilon}_{1} \approx \frac{1}{2}\left(\phi_{0} / 4 \pi \lambda\right)^{2}$ so we have $\widetilde{\varepsilon}_{1} \sim \varepsilon_{1 M}$ in this highly layered limit. Thus, even if one totally eliminates hopping between layers, the long-length scale stiffness of an isolated vortex line is only reduced by a factor of $\ln \kappa_{\perp}$ and $\Delta H_{f} / H_{c 1}$ remains of order $\left(\lambda / \Lambda_{T}\right)^{2}$.

The above analysis is made ignoring demagnetization effects. Although $\Delta H_{f} / H_{c 1}$ is so small, the actual spac- ing between vortices at the melting point is roughly $a_{v} \approx \lambda\left|\ln \left(\Delta H_{f} / H_{c 1}\right)\right|$, so it may only be, say, ten times $\lambda$. Thus, this reentrant vortex fluid phase near $H_{c 1}$ might be accessible in a highly planar sample since, in this case, $\mathbf{B} \simeq \mathbf{H}$ even at very small fields and one can readily control the vortex spacing $\left(\propto B^{-1 / 2}\right)$ with the applied field. ${ }^{43}$ The low-field melting transition will occur at

$$
B_{M} \sim \frac{\phi_{0}}{\lambda_{\perp}^{2} \ln ^{2}\left(\Lambda_{T} / \lambda_{\perp}\right)},
$$

which reaches a maximum at $\simeq \frac{1}{2} T_{c}$. This is shown schematically in Fig. 4.

\section{B. Vortex-lattice melting at higher fields}

As discussed in the previous subsection, in a disorderfree superconductor, a vortex-fluid phase will intrude between the Meissner phase and the vortex-lattice phase ${ }^{6,15}$ as the field is increased from $H_{c 1}$. For a strongly type-II material with $\kappa \gg 1$, there is a wide range of fields between the point at which a vortex lattice will form $\left(B \sim \phi_{0} / \lambda_{1}^{2}\right)$ and $H_{X}=\phi_{0} /\left(2 \pi \xi^{2}\right)$ where the vortex cores strongly overlap. However, as the field is increased towards $H_{X}$, the lattice becomes soft due to the depression of the order parameters and can melt well before $H_{X}$. This transition has been examined in Refs. 6, 7, 16, and 17.

The true phase boundary from vortex lattice to fluid will occur at some melting temperature $T_{M}(H)$, whereas only a smooth crossover from a vortex fluid to completely normal-state behavior will occur in the vicinity of the mean-field transition temperature $T_{c 2}^{\mathrm{MF}}$. We consider $\mathbf{H}$ parallel to the $\hat{z}$ axis in the range $H_{X} \gg H \gg H_{c 1}$, so that the finite size of the vortex lines can be ignored. Houghton et al. ${ }^{16}$ and Brandt ${ }^{17}$ have also obtained some of the results in this region and further results for $H$ near $H_{c 2}^{\mathrm{MF}}$ in the Ginzburg-Landau regime where $H_{X} \simeq H_{c 2}^{\mathrm{MF}}$. There are two sources of anisotropy which both play important roles in this intermediate field regime: the intrinsic anisotropy $\gamma=\lambda_{1} / \lambda_{z}<1$ and the anisotropy imposed by the magnetic field.

Following other authors, ${ }^{6,7,16,17}$ we estimate the mean-square displacement of a single vortex line $W \equiv\left\langle\mathbf{u}^{2}\right\rangle$ due to thermal fluctuations. A perfect static lattice is, as usual, used as the reference state from which $\mathbf{u}$ is the displacement. A simple crude estimate of the melting temperature is given by a Lindemann-like criterion that the root-mean-square (rms) displacement be some fraction $c_{L} \cong 15 \%$ of the lattice constant $a_{v} \equiv\left(\phi_{0} / B\right)^{1 / 2}$, i.e., $\quad W \simeq\left(c_{L} a_{v}\right)^{2}$. Since, in twodimensional or quasi-two-dimensional regimes, $\left\langle\mathbf{u}^{2}\right\rangle$ diverges logarithmically with system size or other cutoff, a better Lindemann criterion for these regimes is that the rms fluctuation in the nearest-neighbor separation be some fraction of the lattice constant.

The elastic properties of an anisotropic flux lattice with magnetic and Josephson coupling between the layers are somewhat complicated and we will not discuss them in detail here. The important moduli are the in-plane shear modulus, which in mean-field theory is ${ }^{44}$ 


$$
\mu=\frac{\phi_{0} B}{64 \pi^{2} \lambda_{\perp}^{2}}
$$

and the tilt modulus $K_{T}(\mathbf{q})$. The bulk modulus for $H \gg H_{c 1}$ is much larger due to the long-range (logarithmic) interaction between the vortices. At long wavelengths the tilt modulus of the vortex lines is dominated by the magnetic energy and equal to $B^{2} / 4 \pi$ for $\mathrm{q} \rightarrow 0$. The wave-vector dependence of the tilt modulus $K_{T}(\mathbf{q})$, is crucial, however, ${ }^{16,17}$ since the dominant thermal fluctuations are generally of short wavelength compared to the penetration length: These short-wavelength fluctuations do not alter the magnetic fields by as much as longwavelength fluctuations and are hence much softer.

The integral over $\mathbf{q}$ which yields the mean-square vortex-line displacement $W$ is generally dominated by $q_{1}$ near the in-plane boundary, but there are several different regimes for $q_{z}$. For $\lambda_{z} / \lambda_{\perp} \gtrsim a_{v} / d$, i.e., very strong anisotropy, the $z$ component of the wavelength of the dominant fluctuations is of order the interplanar separation $d$ so that the system has essentially two-dimensional fluctuations. In this limit, the system is best thought of as consisting of interacting point vortices in each layer, rather than vortex lines. Here we straightforwardly obtain

$$
W \approx \frac{T}{4 \pi \mu d} \ln \left(\frac{B \lambda_{\perp}^{2}}{\phi_{0}}\right),
$$

which is just the $2 \mathrm{D}$ result ${ }^{44}$ for uncoupled layers of thickness $d$ with the large-length scale fluctuations cut off by the range of the magnetic interactions $\lambda_{1}$.

In this regime, the melting temperature should be only slightly higher than the melting temperature of a $2 \mathrm{D}$ system of logarithmically interacting point vortices which has been studied earlier by one of us. ${ }^{44}$ For this $2 \mathrm{D}$ Kosterlitz-Thouless-type melting transition,

$$
T_{M}=\frac{d \mu\left(T_{M}\right)}{2 \pi \sqrt{3}} \frac{\phi_{0}}{B} .
$$

Interplanar coupling, present in the layered system, will tend to increase the melting temperature from (5.4) slightly (the transition will occur, roughly speaking, when the interplanar coupling over the $2 \mathrm{D}$ correlation area becomes of order $T$ ), but softening of the in-plane longitudinal phonon modes due to bulk screening which causes a finite compressibility of the vortex lattice will tend to lower $T_{M}$. Ignoring these offsetting and, in this $2 \mathrm{D}$ regime with large $\kappa$, small effects, (5.4) gives an estimate of the melting transition for the stacked system. Putting in the mean-field shear modulus and estimating the renormalizations due to nonlinear phonon and vortex interactions to reduce $\mu$ by a factor between 0.4 and 0.8 yields ${ }^{44}$

$$
\begin{aligned}
T_{M}^{2 \mathrm{D}} & \simeq(1-2) \times 10^{-2} \frac{\phi_{0}^{2} d}{16 \pi^{2} \lambda_{\perp}^{2}} \\
& \simeq(20-40 \mathrm{~K})\left(\frac{1000 \AA}{\lambda_{\perp}\left(T_{M}\right)}\right)^{2}\left[\frac{d}{10 \AA}\right) .
\end{aligned}
$$

Note the leading field dependence of the shear modulus is $\mu \sim B$, so $T_{M}$ will be only weakly dependent on field in this 2D regime. A gradual drop of $T_{M}$ in proportion to the suppression of the mean-square local order parameter $\left\langle|\psi|^{2}\right\rangle$ is to be expected for increasing field. The condition for validity of this $2 \mathrm{D}$ regime is that

$$
H_{X} \gg B \gg \max \left\{\frac{\phi_{0}}{d^{2}}\left[\frac{\lambda_{1}}{\lambda_{z}}\right]^{2}, \frac{\phi_{0}}{\lambda_{\perp}^{2}}\right\} .
$$

The Kosterlitz-Thouless melting condition is roughly equivalent to a mean-square nearest-neighbor displacement of $0.03 a_{v}^{2}$.

For less anisotropic materials or for lower fields, the dominant fluctuations in the vortex lattice have wave vectors

$$
q_{z} \sim \frac{\lambda_{z}}{\lambda_{\perp}}\left(\frac{B}{\phi_{0}}\right)^{1 / 2}
$$

so that the "effective lattice constant" in the $z$ direction is a factor of $\gamma$ smaller than the in-plane lattice constant $a_{v}$. In this $3 \mathrm{D}$ regime we find for $B \gg H_{c 1}$,

$$
W \simeq \frac{1}{4} \frac{\lambda_{z}}{\lambda_{\perp}}\left(\frac{\phi_{0}}{B}\right)^{1 / 2} \frac{16 \pi^{2} \lambda_{\perp}^{2} T}{\phi_{0}^{2}} .
$$

The prefactor may actually be somewhat less due to nonlinear effects. Using this result and the Lindemann criterion yields

$$
T_{M} \simeq 4 c_{L}^{2}\left[\frac{\lambda_{\perp}}{\lambda_{z}}\right]\left[\frac{\phi_{0}}{B}\right]^{1 / 2} \frac{\phi_{0}^{2}}{16 \pi^{2} \lambda_{\perp}^{2}},
$$

similar to Brandt $^{17}$ and Houghton et al. ${ }^{16}$ With $\lambda_{z} / \lambda_{1} \approx 50, d \simeq 15 \AA$, and $\lambda_{1}(0) \simeq 1500 \AA$ appropriate for $\mathrm{Bi}-\mathrm{Sr}-\mathrm{Ca}-\mathrm{Cu}-\mathrm{O}$, the crossover between the two regimes will occur for $B$ of order $3 \times 10^{3} \mathrm{G}$. For an even more anisotropic material, the crossover from $2 \mathrm{D}$ to $3 \mathrm{D}$ may occur so close to $H_{c 1}$ that (5.8) would have no regime of validity.

As the field is decreased towards $H_{c 1}$ below this crossover, the interplanar magnetic coupling makes the system more three dimensional and the melting temperature thus increases towards $T_{c}$. However, when $B$ drops below $\phi_{0} / \lambda^{2}$, the in-plane interaction between vortices falls off and $T_{M}$ presumably starts decreasing, eventually matching onto (5.1), as is illustrated in Figs. 4 and 6. Thus, the maximum in $T_{M}$ occurs for $B \simeq \phi_{0} / \lambda_{\perp}^{2}$ where $T_{M}$ is roughly given by

$$
\begin{aligned}
\lambda_{\perp}\left(T_{M}\right) & \simeq 0.1 \frac{\gamma \phi_{0}^{2}}{16 \pi^{2} T_{M}}=0.1 \gamma \Lambda_{T_{M}} \\
& \simeq 2 \gamma\left(\frac{100 \mathrm{~K}}{T_{M}}\right) 10^{-3} \mathrm{~cm},
\end{aligned}
$$

using $c_{L} \simeq 0.15$. For a highly anisotropic material such as $\mathrm{Bi}-\mathrm{Sr}-\mathrm{Ca}-\mathrm{Cu}-\mathrm{O}$, this maximum in $T_{M}(H)$ may occur a few degrees below $T_{c}$. Note that, up to a constant, Eq. (5.9) is the same criterion as the crossover to the magnetic fluctuation critical regime Eq. (4.9).

The expression (5.8) for the melting temperature is 
only valid for $B<H_{X}\left(T_{M}\right)$. As shown by Brandt ${ }^{17}$ and Houghton et al. ${ }^{16}$ there is an extra factor of $\left(1-B / H_{c 2}^{\mathrm{MF}}\right)^{3 / 2}$ on the right-hand side of Eq. (5.8) in the Ginzburg-Laudau regime when $B$ is not much smaller than $H_{c 2}^{\mathrm{MF}}$ due to the reduction of the local order parameter and the strong overlap of the vortex cores. The condition that melting occurs at $B_{M}(T)<<H_{X}$ is readily seen, by comparing (5.8) to (4.1), to be equivalent to being in the $X Y$ critical regime, implying that (5.8) as written only applies in the $X Y$ critical regime. Indeed, putting the relation between $\lambda_{\perp}$ and $\xi_{\perp}$ from Eqs. (4.4)-(4.6) into (5.8), we obtain an upper melting field of

$$
B_{M} \simeq 0.1 H_{X} \simeq 0.1 \frac{\phi_{0}}{2 \pi \xi^{2}} \sim\left(T_{c}-T\right)^{2 v},
$$

so that, in this regime, $B_{M} \ll H_{X}$ and the corrections due to overlap between the vortex cores should be minor.

At lower temperatures, outside of the $X Y$ critical regime, there are two possibilities. First, if the critical region is small, as for $\mathrm{Y}-\mathrm{Ba}-\mathrm{Cu}-\mathrm{O}$, the melting curve will approach $H_{c 2}^{\mathrm{MF}}(T)$ as the temperature is lowered. For $\mathrm{Bi}-\mathrm{Sr}-\mathrm{Ca}-\mathrm{Cu}-\mathrm{O}$, on the other hand, where the $X Y$ critical region should be wider, the behavior at intermediate temperature can be given by the quasi-2D result of Eq. (5.5). We will not work out details of the crossover here. The behaviors are illustrated schematically in Figs. 2 and 5.

Note that all the constants in this section, with the exception of those in Eqs. (5.2)-(5.5), are quite uncertain: $c_{L}$ could be considerably less than the value of 0.15 we have used, although $c_{L} \simeq 0.15$ is consistent with the $2 \mathrm{D}$ limit if we use, instead, the equivalent nearest-neighbor displacement. Indeed, any estimate of melting temperature using the Lindemann criterion is very roughessentially just amounting to a balance of thermal and elastic free-energy scales. A proper theory of melting must take into account the effects of dislocations which may be rather different for such intrinsically anisotropic systems than for the conventional solids for which the Lindemann criterion empirically works rather well. In addition, it is possible that, in a disorder-free system, an intermediate 3D hexatic phase might occur between the vortex lattice and the isotropic fluid phase ${ }^{42}$ as occurs in some liquid crystals. We leave these problems for future investigations.

\section{DISORDER AND PINNING}

So far we have mostly considered perfectly clean superconductors. In such an idealized system the only phase transitions which are readily observable in transport measurements are those into the Meissner phase at $H=0$ and $T=T_{c}$, or at $H_{c 1}(T)$. As mentioned earlier, the vortex melting transitions occurring for $H>H_{c 1}$ will not strongly affect the resistive behavior (except, perhaps, for currents parallel to $\mathbf{B}$ ) since, in the absence of disorder, there will be a linear response to an applied current determined by the vortex mobility which will not be strongly affected by a melting transition. Thus, in the absence of pinning, the system will not be a real superconductor for $H>H_{c 1}$, instead having nonzero Ohmic resistivity.
Any real material is, of course, imperfect and the imperfections will tend to pin the vortices, particularly at low temperatures below the clean system's lattice freezing temperature. As stated at the beginning of the previous section, we will restrict our detailed discussion to the effects of microscopic inhomogeneities where much of the physics should be independent of the details of the pinning. Macroscopic inhomogeneities may, however, be more important for obtaining large critical currents. Intermediate scale disorder, particularly microscopic $(\sim 100-\AA$ scale $)$ twin boundaries, will play a role qualitatively similar to microscopic defects, as long as their spacing is less than or of order the intervortex spacing. We will not make quantitative estimates of their effects here, although (see Sec. XI) they may well be important in some experiments on cuprate superconductors.

A vortex line will feel a locally random potential $V(\mathbf{r})$ which arises from interaction with impurities or other local defects (probably oxygen vacancies, interstitials, or microscopic twin boundaries in the cuprate superconductors). This will act primarily on the vortex core and is hence referred to as core pinning; larger-scale defects also couple to the currents around the core. For a vortex line running roughly in the $z$ direction with transverse position $\mathbf{r}_{\perp}(z)$ at height $z$, the pinning free energy is

$$
F_{P}=\int d z V\left(\mathbf{r}_{\perp}(z), z\right),
$$

where the random potential $V(\mathbf{r})$ is due to all the impurities within of order a vortex core size $\xi_{\perp}$ from the vortex center.

As argued by Larkin and Ovchinikov, ${ }^{9}$ the random forces due to even a weak-pinning potential will cause divergent distortions of the vortex lattice at large distances. This is a result of the competition of the energy due to the random potential which, in an only slightly distorted volume of $L^{D}$, grows as $\left(\Gamma L^{D}\right)^{1 / 2}$, and the elastic energy $\sim K L^{D-2}$ of the displacements of order a lattice constant which are necessary to optimize the disorder. Here $K$ is a characteristic elastic modulus and $\Gamma$ is a measure of the mean-square pinning potential. For any $D<4$, the disorder will dominate for length scales larger than a pinning length $L_{P}$ which scales as $L_{P} \sim\left(K^{2} / \Gamma\right)^{1 /(4-D)}$ for weak disorder (small $\Gamma$ ). On length scales up to $L_{P}$, the system will look like a lattice while, on larger scales, the lattice positional correlations will be lost. How drastic will this destruction of the lattice order be? The conventional calculation ${ }^{45}$ includes only the effects of elastic strains; however, on length scales larger than $L_{P}$, dislocations will also appear. This can be seen by considering the elastic energy of a dislocation loop (or equivalently a dislocation pair in 2D) of size $L$. Up to logarithms, this energy is also of order $K L^{D-2}$. Because the dislocation loop will induce displacements of order a lattice constant in a volume of size $\sim L^{D}$, the potential gain in pinning energy is again $\left(\Gamma L^{D}\right)^{1 / 2}$ so that dislocations will become energetically favorable on length scales greater than $L_{P}$. Thus, on scales larger than $L_{P}$, the vortex-lattice description breaks down completely and one is faced with either a fluid or a new nonfluid phase: the vortex-glass phase. Nevertheless, if the disor- 
der is weak, the lattice order will persist out to longlength scales and the energetics of the lattice fluctuations without disorder will set the overall scale of the thermal fluctuations.

Before studying the crossover due to pinning further, we make some rough estimates of the effects of the pinning in various regimes of the phase diagram. If we assume the defects are randomly distributed, the most important quantity is the correlation function of the deviation of $V$ from its mean value, which we take to be zero. In Fourier space, we define

$$
\overline{V(\mathbf{q}) V\left(\mathbf{q}^{\prime}\right)}=(2 \pi)^{D} \delta\left(\mathbf{q}+\mathbf{q}^{\prime}\right) \Gamma_{p}(\mathbf{q}),
$$

where the overbar denotes an average over the random potential. Surprisingly, in most of the strongly fluctuating regimes of interest, the vortex core size, which cuts off $\Gamma_{p}(q)$ for high $q$, will drop out and only $\Gamma_{0} \equiv \Gamma_{p}(\mathbf{q}=0)$ will be important. For random impurities with one site per unit cell of size $a^{2} d$ and fractional concentration $n_{I}$, we estimate from the Ginzburg-Laudau theory that $\Gamma_{0}$ is roughly temperature independent and given by

$$
\Gamma_{0} \approx \frac{1}{4} \gamma_{I}^{2}\left(a^{2} d\right) n_{I}\left(\frac{T_{c}}{T-T_{c}}\right)^{2}\left(\frac{\phi_{0}}{4 \pi \lambda_{\perp}}\right)^{4},
$$

where

$$
\gamma_{I}=\frac{d \ln T_{c}\left(n_{I}\right)}{d n_{I}}
$$

is a dimensionless coupling constant to the impurities and we have estimated the numerical prefactor. Note that the effects of averaging the potential over the core volume $\left(\sim \xi^{2} d\right)$ and the temperature dependence of the order parameter yield the expression (6.3) for $\Gamma_{0}$ which depends only on $\lambda$ (within the Ginzburg-Landau theory), since the singular part of the energy density which couples to the impurities,

$$
\sim d\left(\phi_{0}^{2} / \lambda^{2} \xi^{2}\right) / d(\ln T),
$$

is related to $\lambda$ and $\xi$ in such a way that $\Gamma_{0}$ is independent of $\xi$.

In the presence of thermal fluctuations, the effects of a weak-pinning potential can be strongly reduced due to motional averaging over the area of the thermal vortexline fluctuations. This will give rise to a reduction of the high- $q_{\perp}$ components of $\Gamma_{p}(q)$ for wave vectors greater than $W^{-1 / 2}$, where $W$ is the mean-square displacement of a vortex line due to short-wavelength thermal fluctuations as calculated in Sec. V. Similar effects have been discussed recently by Feigel'man and Vinokur ${ }^{46}$ and Inui et al. ${ }^{5}$

Other types of randomly spaced defects with high enough density will give rise to similar behavior but with a different $\Gamma_{0}(T)$. If the spacing or size of the defect is larger in-plane than the size of the thermal fluctuations of a vortex line $\sqrt{W}$ or larger than $\gamma a_{v}$ in the $z$ direction, the behavior will change somewhat, giving rise to different temperature and field dependences. Large-scale defects such as weak-link grain boundaries will yield qualitatively different behavior; we will not explore these more macroscopic types of pinning in detail here.

\section{A. Effects of pinning on the vortex lattice}

The effects of the pinning can be estimated in a vortexlattice phase by calculating the relative displacements due to the pinning of vortices a distance $\mathbf{r}$ apart. We define a fractional mean-square displacement

$$
\Delta_{D}(\mathbf{r})=\frac{B}{\phi_{0}} \overline{[\mathbf{u}(\mathbf{r})-\mathbf{u}(0)]^{2}} .
$$

A particularly useful measure of the strength of the disorder is the mean-square relative displacements of nearestneighbor (NN) vortex lines, $\Delta_{D}^{N N}=\Delta_{D}\left(\mathbf{r}_{\perp}=\mathbf{r}_{\mathrm{NN}}, z=0\right)$ For relatively weak disorder, we can estimate $\Delta_{D}$ from linearized elastic theory. The dominant Fourier components of the random potential are those near reciprocal-lattice vectors $\mathbf{G}_{\perp}$. However, thermal fluctuations of the vortex lines will smear out the random potential over length scales $\sim W^{1 / 2}$ thus cutting off the high- $G_{\perp}$ Fourier components of $V$. The mean-square pinning force per unit length on a single vortex line $f_{P}$, which is what determines the displacements, is given by

$$
\overline{\left\langle f_{P}^{2}\right\rangle} \propto \int_{Q} Q^{2} \Gamma_{p}(Q) .
$$

But the thermal fluctuations cut off this integral at high momenta $Q_{\perp} \gtrsim W^{-1 / 2}$ yielding $\overline{\left\langle f_{P}^{2}\right\rangle} \sim \Gamma_{0} / W^{2}$. For temperatures high enough that $W>\xi_{1}^{2}$, we then obtain

$$
\Delta_{D}(\mathbf{r})=\left[\frac{B}{\phi_{0}}\right]^{2} \int_{\mathbf{q} \varepsilon B Z} \operatorname{Tr}[\overleftrightarrow{\Lambda}(\mathbf{q})]^{-2} \frac{\Gamma_{0}}{2 \pi W^{2}}(2-2 \cos \mathbf{q} \cdot \mathbf{r}),
$$

where $\overleftrightarrow{\Lambda}(\mathbf{q})$ is the elastic tensor. Since this is proportional to $q^{2}$ for small $q, \Delta_{D}(\mathbf{r})$ diverges for large $r$ in $D<4$. However, due to the strongly wave-vector-dependent nature of the vortex elasticity, the detailed distance dependence of $\Delta_{D}$ will be rather complicated. When the relative distortions become of order a lattice constant, linear elasticity theory, and thus (6.6), is no longer valid; this occurs at length $L_{P}$. (See, however, the discussion at the end of Sec. VII.)

We now estimate $\Delta_{D}^{\mathrm{NN}}$ in various regimes to gauge the strength of the pinning. We will ignore numerical coefficients of order unity. This is simplest in the quasitwo-dimensional intermediate field regime where the layers are weakly coupled and the vortices are logarithmically interacting within each layer. In this case, the only interesting field and temperature dependences arise from the motional averaging of the random potential over an area of order $W$ from Eq. (5.3), ignoring the effects of the long-wavelength contributions to $W$. This gives rise to

$$
\Delta_{D}^{\mathrm{NN}} \sim \frac{B d}{\phi_{0}} \frac{\Gamma_{0}}{T^{2}}
$$

which will apply provided $W>\xi_{\perp}^{2}$ (or the scale of the defect separations) and the disorder is weak $\left(\Delta_{D}^{\mathrm{NN}}<1\right)$. We thus find that, at fixed temperature, high enough that $W>\xi_{1}^{2}$, the effects of the disorder are weaker for small fields provided the system is in the lattice phase. The lat- 
tice correlation length $L_{P} \sim T^{2} / B$ in this quasi- $2 D$ regime. For numbers appropriate to $\mathrm{Bi}-\mathrm{Sr}-\mathrm{Ca}-\mathrm{Cu}-\mathrm{O}$, $\gamma \simeq 0.02$ and $\lambda_{1}(0) \simeq 1500 \AA$, for $B=10^{4} \mathrm{G}$ and $T=30 \mathrm{~K}$, we obtain a rough estimate $\Delta_{D}^{\mathrm{NN}} \approx 0.2 \gamma_{I}^{2} n_{I}$ from impurities which indicates that the effects of disorder can be relatively strong with reasonable impurity coupling.

In the $3 D$ regime with $H_{c 2} \gg B \gg H_{c 1}$ and the Josephson coupling between the layers dominant, the thermal smearing has a weaker effect yielding

$$
\Delta_{D}^{\mathrm{NN}} \simeq \frac{1}{10} \frac{\lambda_{\perp}}{\lambda_{z}}\left(\frac{B}{\phi_{0}}\right)^{1 / 2} \frac{\Gamma_{0}}{T^{2}},
$$

again provided $W>\xi_{\perp}^{2}$ and $\Delta_{D}^{\mathrm{NN}}<<1$. In this regime, the $B$ dependence of $L_{P}$ is complicated, but at fixed $T$ the lattice will have longer-range correlations for smaller $B$.

\section{B. Pinning near the melting transition}

It is useful to obtain a naive estimate of the effects of the disorder near the melting temperature of the clean system, assuming it is first order so that the reduction near the transition of the elastic constants, etc., is not too large.

(1) Intermediate fields. As mentioned earlier, the regime where the upper melting line $B_{M} \ll H_{X}$ is restricted to the $X Y$ critical region. Here we can estimate $\Gamma_{0}$ by merely using the appropriate form Eq. (4.5) for $\lambda(T)$ in Eq. (6.3); it can be readily checked that this has the correct temperature dependence. Doing this, one finds in the $X Y$ critical region that, for impurity coupling,

$$
\Delta_{D}^{\mathrm{NN}}\left(B=B_{M}\right) \sim \gamma_{I}^{2} n_{I}\left(\frac{a^{2} d}{\gamma \xi_{0 \perp}^{3}} \varepsilon_{x}^{-1 / 2}|\varepsilon|^{3 v-2}\right),
$$

with the width of the critical region $\varepsilon_{x}$ given by Eq. (4.8) and $\varepsilon \equiv\left(T-T_{c}\right) / T_{c}$. The coefficient in Eq. (6.9) is likely to be quite small, but it will be enhanced by softening of the lattice. Note that, in this regime, the thermal fluctuations of a vortex line are still somewhat bigger than (although of order) $\xi_{\perp}$ so that Eq. (6.6) still applies. As should have been anticipated for $v=\frac{2}{3}, \Delta_{D}^{\mathrm{NN}}$ is independent of the reduced temperature $\varepsilon$. This is just because disorder is marginal at the zero-field transition for $v=2 / d$. The factor

$$
\frac{\varepsilon_{x}^{-1 / 2}}{\gamma \xi_{0 \perp}^{3}}=\frac{1}{\widetilde{\xi}_{0 \perp}^{2} \widetilde{\xi}_{0 z}} \equiv \widetilde{v}_{c}^{-1}
$$

is just the critical amplitude of the inverse correlation volume. Not surprisingly, $\Delta_{D}^{\mathrm{NN}}$ is just proportional to the ratio of the impurity volume to $\widetilde{v}_{c}$. For $\mathrm{Y}-\mathrm{Ba}-\mathrm{Cu}-\mathrm{O}$, with $\xi_{01} \simeq 15 \AA$, the expression in parentheses in Eq. (6.9) is of order one, which suggests that the lattice correlations may not extend to very long distances near melting, as we discuss further below - although there are clearly large uncertainties in this estimate and the actual behavior can vary strongly from sample to sample, depending on the type of density of defects.

If the field is scaled by $\phi_{0} / \xi^{2}$ then, in the $X Y$ critical regime, the effects of microscopic disorder are independent (up to logarithmic corrections) of the reduced temperature. Thus, all manifestations of the disorder, in particular, the vortex-glass transition which we discuss in Sec.
IX, will scale in this same way. The effects of the disorder will only become logarithmically weaker as $T_{c}$ is approached due to the marginal irrelevance of disorder at the 3D $X Y$ critical point. This will also obtain for other types of defects provided the spacing between them is smaller than the correlation lengths, $\xi_{\perp}$ and $\xi_{z}$ in this regime.

(2) High fields. At low temperatures for which $B_{M}$ is near $H_{c 2}^{\mathrm{MF}}$, the softening of the lattice due to vortex overlap needs to be taken into account. A simple estimate yields $\Delta_{D}^{\mathrm{NN}}\left(T_{M}\right) \sim 1 / T_{M}$ in this regime; thus, the effects of the disorder become larger as $B$ increases and $T_{M}$ decreases.

In general, then, along the higher-field melting line, we expect the effects of disorder to gradually grow as the field is increased, perhaps most substantially as the melting field grows from its value given by (5.10) in the $X Y$ critical region towards $H_{c 2}^{\mathrm{MF}}$ as the temperature is lowered.

(3) Small fields. In small enough fields such that the vortices are far apart compared to $\lambda_{\perp}$, the effects of the pinning can be analyzed in terms of pinning of individual lines. This will tend to pin the flux lines even in the fluid regime, and a relatively small amount of disorder should be sufficient to turn the low-temperature part of the lowfield reentrant fluid phase that is illustrated in Figs. 4 and 6 into part of the vortex-glass phase. A simple guess is that the effects of disorder on the melting transition will be weakest in this low-field regime when $T_{M}$ is closest to $T_{c}$. We leave more detailed investigation of this regime for future work. The effects of thermal averaging of the pinning due to fluctuations of the individual vortex lines will need to be taken into account. ${ }^{5,46}$

\section{CROSSOVER FROM VORTEX LATTICE TO A PINNED PHASE}

We now consider how the crossover from a vortex lattice to a phase dominated by pinning will manifest itself for weak disorder, both within the vortex-lattice phase and near to the melting transition. At this point we will not presume that the resulting phase which describes the long-wavelength behavior is a vortex glass; it could instead be a strongly correlated pinned vortex fluid. We will return to this issue in the next section; here we consider the effects of disorder on length scales where a lattice description is still appropriate.

In the presence of an applied current $J$ normal to the vortex lines, a bulk force is exerted on all the flux lines. In a volume $L_{P}^{D}$, motion of the vortices by one lattice constant in a direction normal to the current will gain an energy $F_{J} \sim J L_{P}^{D}$ from the current. When this is of order the pinning energy in the correlation volume $F_{P} \sim L_{P}^{D-2}$, the flux lines will start to flow. ${ }^{9}$ This yields the fluctuationless weak-pinning critical current: $J_{c}^{P} \approx J_{F} \sim L_{P}^{-2}$ which will characterize the onset of strong nonlinearities in the current-voltage characteristics. Of course, with thermal fluctuations, the dissipation will be nonzero for $J<J_{F}$ due to thermal creep of bundles of flux, which we will discuss in more detail below. As in the Meissner phase, a measure of the strength of the fluctuations is ob- 
tained by estimating the activation barrier for flux creep at a current density of order but less than the fluctuationless critical current density. The simplest guess is that this barrier scales as the pinning energy $F_{P}$ in the volume $L_{P}^{D}$. For weak disorder in three dimensions, $F_{P}$ will be much larger than $T$ so any additional rounding of the $I-V$ curve due to thermal fluctuations will be small. (Note that, if the barriers grow more rapidly than pinning energies, ${ }^{14}$ the rounding will be further reduced.)

If the behavior is quasi-two-dimensional, on the other hand, $F_{P}$ does not grow with $L_{P}$ (except perhaps logarithmically) so that the barriers will be smaller near $J_{F}$ and thermal fluctuations may affect the $I-V$ curve significantly at temperatures of order $T_{M}$. This is no surprise, since 2D systems generally tend to exhibit fluctuation effects much more strongly than their 3D counterparts.

We now consider the effects of weak pinning near to the disorder-free flux-lattice melting transition temperature. The behavior depends crucially on the nature of the melting transition in a completely clean system, i.e., on whether it is first or second order. If, as appears likely in three dimensions, ${ }^{47}$ the melting transition is first order, then we expect that, with weak enough pinning, it will remain so. In this case, there should be no large barriers above $T_{M}$ and the resistivity will not be thermally activated. Immediately below $T_{M}$, however, the $I-V$ curve will suddenly become strongly nonlinear at low currents although at currents higher than several times the fluctuationless critical current $J_{F}$, it will change by very little. Thus, there will not be a gradual decrease of the linear resistivity to very small values; The linear resistivity will drop discontinuously at $T_{M}$ by at least a factor of $e^{-F_{P}\left(T_{M}\right) / T_{M}}$ and probably-if the low-temperature phase is indeed a vortex glass - all the way to zero. Macroscopic inhomogeneities can, of course, round out this behavior - they would result in a percolationlike superconducting transition which should be identifiable.

If the pure system's melting transition is continuous, on the other hand, the behavior will be rather different. In this case, the elastic moduli of the lattice will vanish for $T \rightarrow T_{M}^{-}$as ${ }^{36} K \sim T_{M} \xi_{L}^{2-D} a_{v}^{-2}$, where $\xi_{L}$ is the correlation length for translational order of the lattice which will diverge as $T_{M}$ is approached. (We are assuming that there is a critical melting transition at $T_{M}$ at which isotropic hyperscaling relations are satisfied.) This implies that, for $L_{P} \gg \xi_{L}$, the characteristic scale of the barriers to move regions of size $L_{P}$ is of order

$$
B\left(L_{P}\right) \sim T_{M}\left(\frac{L_{P}}{\xi_{L}}\right)^{D-2} .
$$

Thus, for $T$ less than but near $T_{M}$, there will only be activation barriers on scales larger than $\xi_{L}$. On smaller scales, and at all scales above $T_{M}$, the system will be fluidlike so that there will be no real barriers, and the dynamics will be primarily determined by conventional power-law critical slowing down. Close enough to $T_{M}$, however, even for arbitrarily weak pinning, $\xi_{L}$ will eventually become comparable to $L_{P}$. This is a consequence of the relevance at the melting critical point of random pinning - which acts like a random field on the lattice order parameter. Because of the reduction of the order parameter, with an exponent $\beta_{L}$, the effective pinning strength $\quad \Gamma \sim\left(T_{M}-T\right)^{2 \beta_{L}}$. This yields, via $L_{P} \sim\left(K^{2} / \Gamma\right)^{1 /(4-D)}$, a pinning length which grows less rapidly than $\xi_{L}$ (or actually decreases) as $T_{M}$ is approached. The crossover to disorder-dominated behavior will occur when $\xi_{L} \sim L_{P}$ at a length scale

$$
L_{X} \sim a_{v}\left(\frac{T_{M}^{2}}{\Gamma_{0}}\right)^{1 /\left(2-\eta_{L}\right)},
$$

with $n_{L}$ the critical correlation function exponent of the melting transition. Thus, for small disorder, the resistivity will only show a large decrease very close to $T_{M}$ once $\xi_{L} \sim L_{X}$. In sum, for weak pinning, the resistive drop at $T_{M}$ will hence be very sharp for both first- and secondorder pure system melting transitions. A gradual drop of the resistivity (e.g., approximately Arrhenius over a significant range) is thus indicative of strong effects of pinning.

It is not a priori clear whether increasing pinning strength will raise or lower the transition temperature $T_{G}$. For very strong pinning, the individual vortex lines will be pinned even in what was the pure system's vortex-fluid phase. Thus, we expect very strong pinning to move the vortex-glass transition up closer to the onset of the vortex-fluid regime at $H_{c 2}^{\mathrm{MF}}$. We note, however, that for both strong and weak pinning, the change in the vortex-glass phase boundary will, in general, depend on the type of pinning.

In the $X Y$ critical regime (as mentioned in Sec. VI above), the effects of disorder will be approximately independent (up to logarithmic factors) of the reduced temperature if the magnetic field is scaled by $H_{X} \sim \phi_{0} / \xi^{2}$. Thus, for fixed pinning strength, the normalized crossover lengths $L_{P} / \xi$ and $L_{P} / a_{v}$ will only depend on the scaled field $H \xi^{2}$ in this regime (at least for fields well above $H_{c 1}$. )

We have implicitly assumed in this section that the correlation length of the vortex pinning potential is less than or of order a vortex lattice constant $a_{v}$. Because of the thermal smearing effects, this will effectively be true near to the melting temperature but may not be true for $T \ll T_{M}$. In this case there is an intermediate lengthscale regime where the relative displacements of the lattice due to pinning are between the core size, $\xi_{1}$, and $a_{v}$. In this regime, for lengths less than $L_{P}, \Delta_{D}(r)$ grows as a nontrivial power of the length scale, barriers will appear, but dislocations are not yet important. We will not discuss this regime further here. Some properties in this intermediate regime have been investigated by other authors. 30,45

\section{VORTEX-GLASS PHASE}

We now turn to the behavior at large-length scales $\left(>L_{P}\right)$ and low temperatures in the presence of a penetrating magnetic field. The fundamental issue here is whether a system of vortex lines pinned by the disorder is, at low temperatures, (i) a fluid with finite linear 
conductivity - albeit one which is strongly impeded by the pinning and thus glassy as in the Anderson-Kim theory ${ }^{3,4}$ - or (ii) a true superconductor with infinite linear dc conductivity. If it is a true superconductor, this would be a new equilibrium phase which we call a vortex glass by analogy with spin glasses (rather than "true" glasses which may well be just nonequilibrium fluids). In this section we discuss some properties of such a putative vortex-glass phase, which we assume exists for temperatures below a transition at $T_{G}(B)$.

A vortex-glass phase will have off-diagonal long-range order of a type analogous to spin glasses: ${ }^{13,14}$ Neglecting gauge fluctuations, the complex scalar pair field $\langle\psi(\mathbf{r})\rangle$ will be nonzero at each point in space (except at the vortex locations, where $\langle\psi(\mathbf{r})\rangle$ vanishes), but its phase will vary from point to point. It is this phase coherence at distances large compared to the range of vortex lattice order $L_{P}$, that makes the vortex-glass phase fundamentally different from that which implicitly appears in the traditional "collective vortex pinning" theories. ${ }^{4}$ In these theories, the possibility of phase coherence on such long-length scales and its effects on vortex dynamics have generally not been considered.

Let us fix the gauge so $\boldsymbol{\nabla} \cdot \mathbf{A}=0$. In this gauge, a correlation function which measures the long-range phase coherence and its onset as the vortex-glass (VG) phase is entered upon cooling, is

$$
G_{\mathrm{VG}}(\mathbf{r})=\overline{\left.\left\langle\psi^{*}\left(\mathbf{r}^{\prime}\right) \psi\left(\mathbf{r}^{\prime}+\mathbf{r}\right)\right\rangle\right|^{2}},
$$

where the angular brackets denote a full thermal average for a given sample and a given $\mathbf{r}^{\prime}$ and the overbar denotes a spatial average over points $\mathbf{r}^{\prime}$. We expect that, in the vortex-glass phase, the correlation function $G_{\mathrm{VG}}(\mathbf{r})$ approaches a nonzero constant as $\mathbf{r} \rightarrow \infty$, whereas, in the fluid phase, $G_{\mathrm{VG}}(\mathbf{r})$ falls off exponentially with the glass correlation length $\xi_{G} \cdot{ }^{48}$

The simplest scenario for the vortex-glass order is that, by analogy with recent theories of spin glasses, ${ }^{14,49}$ there is a unique equilibrium state up to gauge changes. The possibility of many states unrelated by symmetry, as in the replica symmetry-breaking solution of the Sherrington-Kirkpatrick model, ${ }^{13}$ will not be considered here. In any case, we believe that arguments similar to those for Ising spin glasses ${ }^{14,49}$ imply that this is unlikely.

Unfortunately, the off-diagonal correlations manifest in (8.1) cannot be probed directly. Vortex-glass order can, however, be revealed indirectly by the system's electromagnetic response, as detailed below. This can be most readily described by focusing on the vortex lines (zeroes of $\psi$ ), "integrating out" the smoothly varying spin-wave (phase of $\psi$ ) background. As we shall see, it is primarily the dynamic correlations of the vortex lines which are sensitive to the transition into the vortex-glass phase. Evidence for vortex-glass order is probably only present in rather subtle properties of purely static vortex-line correlations, in contrast to the vortex-lattice phase in the pure case in which the static correlations directly exhibit the broken translational symmetry. Indeed, the global $U(1)$ rotational symmetry of the phase of the field $\psi(\mathbf{r})$, which is broken in the vortex-glass state, leaves the positions of the vortex lines unchanged. Of course, when pinning is present, the thermally averaged vortex density will be spatially inhomogeneous in both the fluid and the vortex-glass phases.

\section{A. Nonlinear current-voltage response}

In the Anderson-Kim theory ${ }^{3,4}$ of flux creep, each vortex line (or bundle of lines) is effectively modeled as a single, approximately independent, zero-dimensional "particle" moving in a random pinning potential with some energy scale $U$ which can depend on the applied current $J$ but remains finite for $J \rightarrow 0$. Thermal activation over the barriers leads to a flux-creep resistivity $\rho \sim e^{-U / T}$ which is nonzero at any positive temperature, even in the limit of zero current. In a bulk superconductor, though, vortex lines are extended one-dimensional objects and it is essential to consider possible length dependence of the energy (or, more precisely, free energy) scale $U$. Indeed, as we shall see below, if the energy grows with length scale $L$, no flux-creep linear in the applied current will be possible and the system will be a true superconductor $(\rho=0)$. To illustrate this point we consider first a single, infinitely long, vortex line in the presence of random pinning.

A single vortex line subject to quenched impurities is formally equivalent to a "directed polymer in a random medium" and has been studied extensively in the recent past. ${ }^{50}$ For the physically relevant $3 \mathrm{D}$ case, the vortex line is believed to be in a disorder-dominated pinned phase for any temperature and pinning strength. (For $D>3$, an unpinned phase is also possible at high temperatures.) The long-length-scale fluctuations of the pinned line are determined by the scaling at a zero-temperature strong-disorder fixed point. It is found numerically ${ }^{50}$ that the transverse displacements $L_{\perp}$ of a vortex-line segment of length $L_{z}$, scale as

$$
L_{\perp} \sim L_{z}^{\zeta}
$$

with an exponent $\zeta \simeq 0.6$, a value larger than that with thermal fluctuations but no pinning, $\zeta_{T}=\frac{1}{2}$. The characteristic free-energy cost in moving a vortex-line segment of length $L_{z}$ is found to grow as $L_{1}^{2} / L_{z} \sim L_{z}^{2 \zeta^{-1}}$, scaling as the elastic energy of the vortex line.

As shown by Feigel'man and Vinokur, ${ }^{46}$ these scaling forms can be used to deduce the response of a single vortex line to an applied current. To this end, consider a set of low-free-energy excitations which consist of displacing a segment of line of length $L_{z}$ by an amount of $L_{\perp}\left(\sim L_{z}^{\xi}\right)$ transverse to both the magnetic field and an applied current, as depicted schematically in Fig. 7. These excitations can be described as oriented vortex loops (see Fig. 7) when viewed with respect to the ground-state configuration. A typical loop with given area $S=L_{\perp} L_{z} \sim L_{\perp}^{\kappa}$ will have free energy which grows with size as

$$
F_{L_{\perp}} \approx \Upsilon L_{\perp}^{\theta_{\perp}}
$$

with $\Upsilon$ a stiffness coefficient and $\kappa=1+(1 / \zeta)$. (Note the exponent $\kappa$ in this section differs from the ratio $\kappa=\lambda / \xi$ used elsewhere in this paper.) By analogy with the drop- 


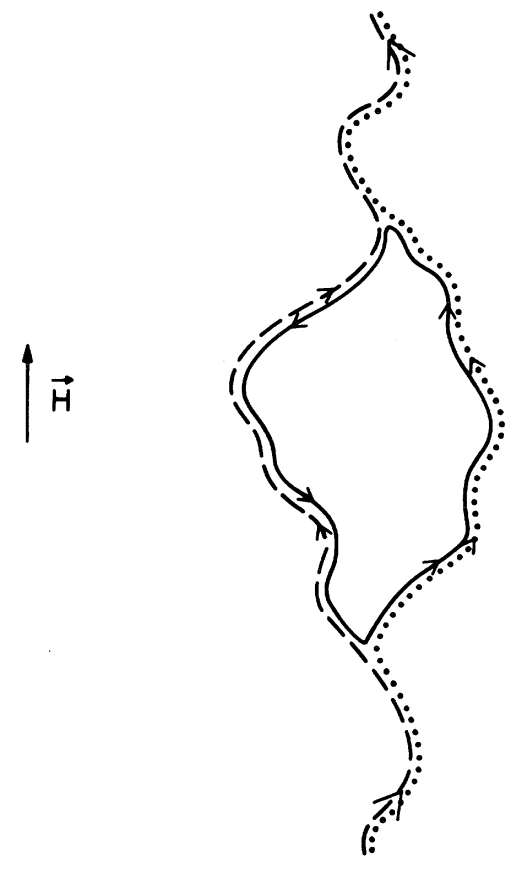

FIG. 7. An excitation of a single pinned vortex line. The dashed line represents the initial configuration, while the dotted line is the final configuration. The excitation may be described as the solid vortex loop, which has been added to the dashed initial configuration, canceling the initial vortex where it is on top of and antiparallel to it, yielding the dotted final configuration. The applied current is assumed to be normal to the paper.

let theory of spin glasses, ${ }^{14}$ we have introduced an exponent $\theta_{\perp}$ to describe the energy dependence as a function of $L_{\perp}$. For the special case of a single vortex-line excitation considered here, $\theta_{\perp}=2-(1 / \zeta) \simeq \frac{1}{3}$.

To obtain these loop excitations from the ground state by continuous deformation, the vortex line will typically have to pass over free-energy barriers $B_{L_{1}}$ which grow at least as fast with area as the loop energies themselves:

$$
B_{L_{\perp}} \sim \Delta L_{\perp}^{\psi_{\perp}}
$$

with an exponent $\psi_{\perp} \geq \theta_{\perp}$. In the presence of an external current $J$, however, the barriers for motion of large sections of line will be reduced since they can be decomposed into motion of a series of smaller sections with length of order $L_{\perp J}$, with each such motion lowering the total free energy. The size $L_{\perp J}$ is determined by balancing the energy gained from the current $\sim J L_{\perp}^{\kappa}$ against $\Upsilon L_{\perp}^{\theta_{\perp}}$. Motion of the vortex line will proceed via thermal nucleation of loops of size $L_{\perp J}$ at a rate proportional to $e^{-B_{L_{\perp J}} / T}$ This motion causes phase slips which, for a model system of many noninteracting pinned vortex lines, corresponds to a steady-state dissipative electric field

$$
E(J) \sim \exp \left[-\left(J_{T} / J\right)^{\mu}\right]
$$

with $\mu=\psi_{\perp} /\left(\kappa-\theta_{1}\right)$ and $J_{T} \sim \Upsilon(\Delta / T)^{1 / \mu}$.

Equation (8.5) indicates that the dissipative motion of even a single randomly pinned vortex line is nonperturbative in the applied current provided it is infinitely long. Thus, it provides no linear resistivity. This should be contrasted with the conventional flux-creep theory. ${ }^{3,4}$

The notion of a vortex-loop excitation can be generalized to the physically relevant case of many interacting vortex lines in a vortex-glass phase. Consider the excitation sketched schematically in Fig. 8, in which a group of vortex lines are effectively translated perpendicular to both $\mathbf{H}$ and an applied current by one intervortex spacing. Such an excitation can also be described as a relative vortex loop as depicted by the solid line in Fig. 8. Indeed, any local excitation of the vortex lines can be described in terms of one or more vortex loops relative to the initial state. In $3 \mathrm{D}$, a vortex-loop excitation is equivalent to moving a plane of vortex lines spanning the loop, each by one vortex spacing (Fig. 8). For simplicity, we will restrict consideration to vortex-loop excitations of size smaller than the "bare" penetration length $\lambda_{s}$ in the absence of vortex motion. On larger scales screening effects may enter; we will not investigate this latter regime in any detail.

Let us denote by $S$ the projected area of a vortex-loop excitation as "seen" by an observer looking along the direction of an applied current, $\mathbf{J}=J \widehat{\mathbf{x}}$. The product $\phi_{0} S J / c$ is then precisely the energy gained from the "magnus force" of the applied current when the loop is created. Of interest are those particular loop excitations which, for given area $S$ and in each given region of the sample, have minimal excitation free energy $F_{L_{\perp}}$ (when $J=0$ ). For a given area $S$ the parallel and transverse scales of the loop should vary, as in (8.2), as $L_{\perp} \sim L_{z}^{\xi}$, with $S=L_{\perp} L_{z} \sim L_{\perp}^{\kappa}$. An exponent $\xi \neq 1$ allows for the possibility of anisotropic scaling in the vortex-glass phase. A

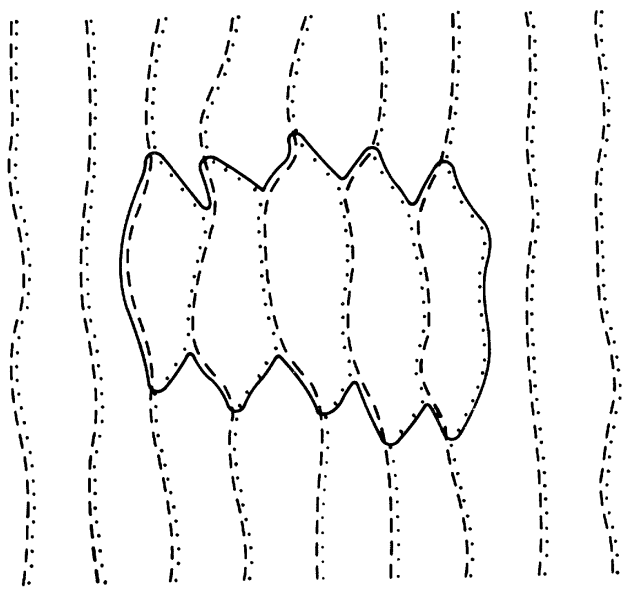

FIG. 8. Excitation of a multiple vortex-line array. As in Fig. 7 , the dashed lines are the initial configuration, the dotted lines are the final configuration, the solid line is the relative vortex loop, and the current is normal to the paper. 
natural ansatz is that $F_{L_{\perp}}$ will scale with the linear size $L_{\perp}$ as in (8.3), except with a different exponent $\theta_{\perp}$. We assume stability of the vortex glass to thermal fluctuations, which requires $\theta_{\perp} \geq 0$, and an upper bound is $\theta_{\perp} \leq \kappa / 2$, which corresponds to a circular loop with nonzero line tension and no relaxation of the other vortices, an excitation that is always possible, as in the Meissner case. Free-energy barriers to create vortex-loop excitations will also presumably scale as in (8.4), with $\psi_{\perp}$ satisfying

$$
0 \leq \theta_{\perp} \leq \psi_{\perp} \leq \kappa / 2
$$

With $J \neq 0$, nucleation of loops with linear size $L_{\perp J}$ such that $J L_{\perp J}^{\kappa} \sim \Upsilon L_{\perp J}^{\theta_{\perp}}$ are likely to be the dominant source of dissipation, causing a phase-slip-induced voltage depending exponentially on current, as in (8.5). Provided that $\theta_{1}>0$, so that the vortex-glass phase is stable at $T \neq 0$, it will be a true superconductor with infinite dc linear response conductivity. The upper bounds on $\theta_{\perp}$ and $\psi_{1}$ in (8.6) give an upper bound on $\mu$, namely $\mu \leq 1$. This bound on $\mu$ arises simply from the fact that one can certainly make circular vortex-loop excitations with free energy proportional to their circumference. Recall that $\mu=1$ in the Meissner phase (3.2) where $\xi=1$ for the loops.

At this point, it is not clear, however, that single vortex-loop excitations, as described above, will always be the dominant nonlinear dissipative process in the vortex-glass phase for small $J$. It is plausible that, instead, 3D bundles of vortex lines might tend to dominate the phase-slip process. Such bundles could be described as a superposition of many vortex loops and would have energies and coupling to current scaling with different exponents $\theta_{\perp}$ and $\kappa$. In any case, we expect the exponential $I-V$ relationship (8.5) to hold at low enough currents, with the properties of the dominant dissipative processes determining $\mu$.

The exponential current-voltage characteristic in the vortex-glass phase leads to an extremely slow relaxation of a remanent (persistent) current (or, equivalently, a remanent magnetization). To be concrete, consider the decay of a persistent current set up by suddenly changing the strength of an applied magnetic field. At extremely short (microscopic) times ( $t \lesssim t_{0} \sim 10^{-9} \mathrm{sec}$ ), the current will decay via a nonactivated, deterministic phase slip, until, at $t_{0}$, it reaches a current density of roughly $J_{F}$. Further decay of current then proceeds by thermally activated vortex-loop excitations at a rate $\partial J / \partial t \sim E(J)$, with $E(J)$ given in (8.5). Integrating with respect to time gives a long-time decay of the form

$$
J(t) \approx J_{T}\left[\ln \left(t / t_{0}\right)\right]^{-1 / \mu},
$$

valid for $J \ll J_{F}$. A reasonable interpolation between the short- $\left(t \sim t_{0}\right)$ and long-time limits is ${ }^{30}$

$$
J(t) \approx \frac{J_{F}}{\left[1+(T / U) \ln \left(t / t_{0}\right)\right]^{1 / \mu}}
$$

with

$$
U / T \approx\left(J_{T} / J_{F}\right)^{\mu}
$$

The form (8.7) should also apply to the decay of a persistent current in the Meissner phase.

When $U \gg T$, on laboratory time scales (8.7) can be replaced by

$$
J(t) \approx J_{F}\left[1-(T / \mu U) \ln \left(t / t_{0}\right)\right],
$$

which, up to constants, is the form predicted by Anderson-Kim flux-creep theory. ${ }^{3,4}$ As emphasized in Secs. III and IV, an essential difference between conventional low $-T_{c}$ superconductors and the high- $T_{c}$ oxides, is that, in the latter, $J_{T}$ is not necessarily much larger than $J_{F}$ even at temperatures well below $T_{c}$. Moreover, in the vortex-glass critical regime near $T_{G}$ (and in the $X Y$ critical regime near $T_{c}$, when $H=0$ ) the ratio $J_{T} / J_{F}$ should be a constant of order 1 . Thus, in the high- $T_{c}$ superconductors, which are expected to have a wide critical regime, it should be possible experimentally to get into the long-time limit of (8.7), $(T / U) \ln \left(t / t_{0}\right) \gtrsim 1$, and check its validity. An essential feature of (8.7) is that for a fixed range of $\ln t, \partial J(t) / \partial \ln t$ is predicted to be a nonmonotonic function of temperature, in contrast to the AndersonKim theory. Such a nonmonotonicity has indeed been observed in $\mathrm{Y}-\mathrm{Ba}-\mathrm{Cu}-\mathrm{O}$ crystals, ${ }^{51}$ although it remains unclear whether this is the correct explanation of those experiments. For temperatures above the crossover temperature, $T_{\mathrm{cr}} \approx U / \ln \left(t / t_{0}\right)$, but below the vortex-glass critical regime where $U$ becomes temperature dependent, $\partial J / \partial \ln t$ should vary as $T^{-1 / \mu}$. To interpret experiments on samples bigger than $\lambda$, an analysis taking into account current inhomogeneities due to screening, is needed. In the linear regime, some such effects are discussed below.

\section{B. Frequency-dependent linear response}

The low-frequency behavior of the linear ac response in the vortex-glass phase is rather subtle. Two simple arguments can be made which are apparently conflicting. Firstly, if the vortices are truly pinned and cannot move, then the vortex glass would be just like the Meissner phase with conductivity

$$
\sigma(\omega) \simeq \frac{\rho_{s}}{-i \omega},
$$

with $\rho_{s}=\rho_{s 0}$ the superfluid density in the absence of vortex motion. Thus, it would have a finite magnetic penetration length

$$
\lambda_{G}(\omega) \sim \frac{1}{\sqrt{\omega|\sigma(\omega)|}}
$$

in the $\omega \rightarrow 0$ limit. We argue below that although $\rho_{s}$ will, in general, be smaller than $\rho_{s o}$, the vortex-glass phase does indeed have a finite penetration length as $\omega \rightarrow 0$. On the other hand, if an additional static field is applied, in equilibrium, extra flux will penetrate the whole of the sample since (at $\omega=0$ ) the limit of $\delta B / \delta H$ as $\delta H \rightarrow 0$ is not zero in the vortex-glass phase. Thus, we appear to have rather different behavior of $\omega=0$ (static field) than is obtained by taking the limit $\omega \rightarrow 0$, and thus a noncommuting of the $\delta H \rightarrow 0$ and $\omega \rightarrow 0$ limits.

We first consider the limit where the Ginzburg-Landau 
penetration length ignoring vortex motion $\lambda_{s}$ is much larger than the intervortex spacing and the crossover length $L_{P}$ from vortex-lattice to vortex-glass behavior. In this limit, we may ignore magnetic fields generated by the screening currents in estimating $\sigma(\omega)$ and then compute the frequency-dependent penetration length from Eq. (8.10).

At any nonzero temperature, the positions of the vortex lines will fluctuate, dissipating an external ac current $J(\omega)$ and reducing the stiffness of the system, i.e., the macroscopic $\rho_{s}$. At high frequencies many vortices can move on small-length scales and, especially near the transition temperature $T_{G}, \rho_{s}$ will be substantially decreased in all of the samples. At low frequencies, however, the behavior is somewhat different. For simplicity let us assume the excitations are indeed relative vortex loops as discussed above, which, moreover, are isotropic, i.e., $\zeta=1$ and $L_{1} \sim L_{z} \sim S^{1 / 2}$ so we may drop the $\perp$ and $z$ subscripts. Only vortex loops of size up to

$$
L_{\omega} \approx\left|\frac{T}{\Delta} \ln \omega\right|^{1 / \psi}
$$

will respond appreciably to a linear ac current with frequency $\omega$, since the barrier for larger loops will be too high to be crossed at rate $\omega$.

Since, in a typical region, the vortex-loop excitations of large size $L$ will have free energy $F_{L} \sim \Upsilon L^{\theta} \gg T$, they will mostly be only very weakly linearly polarized by an external current and can be ignored. Note, however, that for any fixed nonzero current $J$, all large excitations will be nonlinearly polarized since $\theta<2$; this gives rise to the nonlinear flux creep discussed in the previous subsection, which is expected to be relatively homogeneous in space. The thermally active excitations of size $L$, on the other hand, i.e., those with free energy $F \lesssim T$, can be polarized by an infinitesimal current. However, only a small fraction of the system will be occupied by such large active excitations, the simplest expectation being that a fraction $T / \Upsilon L^{\theta}$ of the excitations of size $L$ will be active, since we expect a constant density of excitation energies for $F_{L} \ll \Upsilon L^{\theta}$. (It is possible that a different exponent $\theta$ could appear here, although there is no obvious reason for the behavior of these excitations to differ in this respect from Ising spin glasses. ${ }^{14}$ ) Thus, the large excitations that respond linearly are dilute so that lowfrequency linear response behavior is expected to be spatially quite inhomogeneous. This has interesting consequences, some of which we will now discuss.

At low frequencies, we can make a quasistatic approximation to obtain the effective long-wavelength stiffness $\rho_{s}(\omega)$. We simply ignore all excitations with barriers larger than $T|\ln \omega|$ (i.e., loops bigger than $L_{\omega}$ ) and assume the smaller excitations are polarized as they would be by an infinitesimal dc current. An overestimate of the effects of the active regions can then be made by replacing each of them by an insulating hole which can carry no supercurrent. But, since large active excitations do not percolate, all this will do is reduce the macroscopic stiffness by an amount of order the volume fraction of the holes. Thus, we conclude that, as $\omega \rightarrow 0$, the linear macroscopic $\rho_{s}$ is nonzero in the vortex-glass phase. At low, but nonzero, frequency there will be corrections of the form

$$
\operatorname{Re} \rho_{s}(\omega) \approx \rho_{s}(\omega=0)\left(1+\frac{C}{|\ln \omega|^{\theta / \psi}}\right),
$$

due to the contributions from loops of scale $L_{\omega}$. From the Kramers-Kronig relations we then obtain

$$
\operatorname{Im} \rho_{s}(\omega) \sim \frac{1}{|\ln \omega|^{1+\theta / \psi}} .
$$

This is smaller than the real part for small $\omega$ thus justifying the quasistatic approximation. In addition to the $\delta$ function at $\omega=0$, the low-frequency behavior of the real part of the conductivity is

$$
\operatorname{Re} \sigma(\omega) \sim \frac{1}{|\omega||\ln \omega|^{1+\theta / \psi}}
$$

yielding more excess dissipation of a low-frequency ac current than occurs even in a dirty Meissner phase (see Sec. III).

If we can treat the vortex-glass system as being homogeneous on scales of order $\lambda_{s}$, then we can obtain the ac penetration length from Eq. (8.10) yielding a finite $\lambda_{G}(\omega)$ as $\omega \rightarrow 0$. At temperatures of order half- $T_{G}, \lambda_{G}(\omega=0)$ will be some not too large multiple of $\lambda_{s}$ since the contribution from small-scale vortex loops will reduce $\rho_{s}$ by an amount of order unity. Near $T_{G}, \lambda_{G}$ will diverge. We discuss its behavior in Sec. IX.

More careful considerations show that, even if we take into account inhomogeneities of the vortex glass on scales of order $\lambda_{s}$ and larger, the ac component of an (infinitesimal) applied magnetic field at a typical point a distance $x$ from the surface will fall off as $e^{-x / \lambda_{G}(\omega)}$ as in a Meissner phase, with $\lambda_{G}(0)$ finite. Nevertheless, because of the inhomogeneities, the results of some experimental measurements will, as for spin glasses, ${ }^{14}$ not reflect this typical behavior.

Let us consider an ac field-penetration measurement through a slab of superconductor of macroscopic transverse dimensions and thickness $D$. The uniform dc background field and temperature are chosen to make the system a vortex glass with typical penetration length $\lambda_{G}(0)$. A very small low-frequency ac field $B(\omega)$ is applied above the slab and the resulting spatially averaged ac penetrating field

$$
B_{p}(\omega) \equiv B(\omega) \chi_{p}(\omega, D)
$$

is measured some distance below the slab.

The important feature here is the measuring of the spatially averaged penetrability $\chi_{p}(\omega, D)$ over a large region (but, of course, not large enough to include the effects of fields which go around, rather than through, the slab). We must thus consider the effects of the rare regions in which the field can penetrate locally. We consider frequencies low enough that $L_{\omega}>\lambda_{G}(0)$. Since we are interested in bulk properties we consider only the limit $D \gg L_{\omega}$; in the opposite limit, a two-dimensional description is needed (see Sec. X). In the absence of a perturbation, there will be inhomogeneous static and fluctuating 
fields near the surface of the slab due to vortices within $\lambda_{s}$ of the bottom surface. Indirect polarization of those vortices by interactions with vortices near the top surface, which directly couple to the applied field, can give rise to a locally penetrating ac field. This occurs where there is an active excitation with characteristic relaxation time $\tau<1 / \omega$ which runs from the top to the bottom surface. A roughly circular loop of size $D$ will typically have a barrier much too large to respond at frequency $\omega$ since $D \gg L_{\omega}$. The probability that the barrier of such a loop will be anomalously small is probably of order $e^{-D^{2}}$. These excitations will thus be so rare that we can neglect them. Elongated loops which can be excited by a number of roughly independent smaller loops, of size $L_{\omega}$, will, on the other hand, play an important role. An elongated loop of length $\sim D$ and width $\sim L_{\omega}$ has a small probability of being active, $P_{D}$, but, if it is active, it has a barrier for creation which is not too large. Such an excitation can cause fields of order $B(\omega)$ to penetrate locally. A crude estimate of $P_{D}$ is as the product of $D / L_{\omega}$ roughly independent parts of each section of length $L_{\omega}$, each with probability $P_{L_{\omega}}$. Since $P_{L_{\omega}}$ will have a power-law dependence on $L_{\omega}, \Upsilon$, and $T$, but probably not $D$ [i.e., $\left.P_{L_{\omega}} \sim T /\left(\Upsilon L_{\omega}^{\theta}\right)\right]$, the dominant dependence on $D$ and $L_{\omega}$ is exponential:

$$
P_{D} \sim \exp \left[-b D /\left(L_{\omega} / \ln L_{\omega}\right)\right],
$$

with $b$ a constant. Thus, averaging over a large region of the slab will give a penetrability which is dominated by these elongated active loops:

$$
\chi_{P}(\omega, D) \sim \exp \left[-D / \bar{\lambda}_{G}(\omega)\right],
$$

with an effective penetration length for the average field which diverges for $\omega \rightarrow 0$ as

$$
\bar{\lambda}_{G}(\omega) \sim|\ln \omega|^{1 / \psi}
$$

[with $\ln (\ln \omega)$ factors ignored]. It thus appears, at least at this level of approximation, that there will be two different measurable penetration lengths: one divergent one, $\bar{\lambda}_{G}(\omega)$, measured from the penetration of the average magnetic field, and the other finite one that can be obtained from the screening of a dc applied current which will be dominated by a surface layer of finite thickness $\lambda_{G}(\omega \rightarrow 0)$. A more detailed analysis of these and related issues will be left for future research.

\section{VORTEX-FLUID-TO-VORTEX-GLASS TRANSITION}

\section{A. Scaling behavior}

Let us now consider the scaling behavior near the transition from the vortex-fluid phase to the vortex-glass phase which, for dimensionality $D=3$, may occur at nonzero temperature in the presence of a penetrating magnetic field and random pinning. At least for strong disorder, this transition is expected to be continuous (second order), in contrast to the vortexlattice-to-vortex-fluid transition in the pure case which may be first order. ${ }^{47}$ The width of the vortex-glass critical regime, however, should depend sensitively on the strength of the disorder. As discussed in Sec. VII, the critical regime will be very small (or nonexistent if the transition is first order) in the weak-pinning limit, when the vortex-lattice order persists out to long scales $L_{P}$. If the transition is continuous, it should nevertheless obey the same scaling laws as in the more accessible strongpinning case. It is possible, however, that for weak enough pinning, a first-order glass-to-liquid transition may occur due to first-order lattice melting in the pure case. We will not analyze this latter possibility here.

As the vortex-glass critical temperature $T_{G}$ is approached, the vortex-glass correlation length $\xi_{G}$, which determines the long-distance behavior of the correlation function (8.1), diverges. Since the system is intrinsically anisotropic, one might expect that correlation lengths parallel and perpendicular to the field would have different critical exponents. However, the replica-matrix field theory appropriate to the static critical phenomena at the vortex-glass transition in high dimensions $(D \gtrsim 6)$, as derived by Lubensky and John, ${ }^{12,52}$ has anisotropies of a sort which can be eliminated by a simple rescaling of lengths. The asymptotic critical regime for $D=3$ thus may well be isotropic.

We then expect a single exponent $v$ describing the divergence of the vortex-glass correlation length via $\xi_{G} \sim\left|T-T_{G}\right|^{-v}$ and a characteristic relaxation time ${ }^{38}$ $\tau_{G}$, which exhibits critical slowing down with $\tau_{G} \sim \xi_{G}^{z}$. (Anisotropic critical scaling with $v_{z} \neq v_{\perp}$ would complicate, but not fundamentally change, the following analysis.) Above the upper critical dimension of the glass transition, $D_{u}=6, v=\frac{1}{2}$, and ${ }^{53} z=4$ as for conventional spin glasses. ${ }^{13}$ In $6-\varepsilon$ dimensions, $v$ and $z$ both increase. ${ }^{54}$ The exponent $z$, however, increases by less than in an Ising spin glass ${ }^{13}$ suggesting that in $3 \mathrm{D}$ it may be less than the Ising value $z_{\text {Ising }} \simeq 6 \pm 1$. Thus, a value in the range 4-7 appears most likely. Note that, if $T_{G}$ is close to $T_{c}$, as occurs in a small applied magnetic field, the temperature range of the vortex-glass critical regime will be reduced (up to logarithmic factors) in proportion to $\left(T_{c}-T_{G}\right)$. Currents, lengths, and frequencies should all be appropriately scaled since $T_{c}$ is really a multicritical point; here we generally assume the applied field is large enough that these considerations may be ignored.

(1) Nonlinear current-voltage response. We now consider the general scaling of current density $J$ and electric field $E$ in terms of the exponents $v$ and $z$. We will consider $\mathbf{E}$ and $\mathbf{J}$ along the $x$ axis (for simplicity, ignoring the possible Hall angle between $\mathbf{E}$ and $\mathbf{J}$ ) with $\mathbf{H} \simeq \mathbf{B}$ along the $z$ axis.

Now since $\mathbf{E}=-\partial \mathbf{A} / \partial t$ and $\mathbf{A}$ enters as an inverse length in (2.1), E presumably scales as $1 /$ (length $\times$ time). The appropriate scaling combination is thus $E \xi_{G}^{(z+1)}$. Similarly, we have $\mathbf{J} \sim \partial f / \partial \mathbf{A}$ and, assuming hyperscaling, $f$ scales as (length) ${ }^{-D}$, suggesting that $J$ scales as (length $)^{1-D}$. The appropriate scaling combination is then $J \xi_{G}^{D-1}$. Thus, we obtain the scaling ansatz

$$
E \xi_{G}^{z+1} \approx \widetilde{\mathscr{E}}_{ \pm}\left(J \xi_{G}^{D-1}\right)
$$

for temperatures above $(+)$ and below $(-) T_{G}$, where $\widetilde{\mathscr{E}}_{ \pm}(x)$ is an appropriate scaling function. This is 
equivalent to the scaling form (4.18) obtained for $\mathbf{H}=0$, except here $z$ is different and the appropriate correlation length is $\xi_{G}$. For the vortex-glass transition, the scaling functions have the same qualitative behaviors as for the zero-field transition (4.18) for large and small arguments, except from (8.5) we have $\widetilde{\mathscr{E}}_{-}(x) \sim e^{-a / x^{\mu}}$ for $x \rightarrow 0$ with $\mu$ possibly less than unity.

At the vortex-glass transition temperature, a powerlaw $I-V$ curve is expected, as in (4.19), with

$$
E \sim J^{(z+1) /(D-1)} .
$$

This can serve as a useful criterion to locate the transition; it has been so employed in Ref. 22. Notice that, at the upper critical dimension $D=6$ where $z=4$, Eq. (9.2) yields Ohmic behavior at $T_{G}$. This is consistent with mean-field theory ${ }^{53}$ for which we expect a linear conductivity that jumps discontinuously to infinity at $T_{G}$, precisely as in the mean-field treatment of the pure $H=0$ normal-to-superconductor transition. ${ }^{2}$

Implicit in this scaling analysis, both above and below, is that measurements are performed in a regime where the current flows through the bulk of the sample, with $J$ essentially uniform, rather than being screened out of the bulk and flowing at the surfaces only. This requires that, above the transition, the screening length

$$
\lambda(\omega) \sim[\omega|\sigma(\omega)|]^{-1 / 2},
$$

with $\omega$ the frequency of measurement, be larger than the sample's smallest dimension (e.g., film thickness). The relevant criteria in the nonlinear regime below the transition are more subtle and we have not investigated them in detail.

(2) Linear conductivity. The low-frequency linear conductivity near the vortex-glass transition should scale as in $(4.11)$, with $\xi$ replaced by $\xi_{G}$. The complex scaling functions $\mathcal{S}_{ \pm}(x)$ entering in $(4.11)$ behave essentially as described there, except with $\ln \omega$ corrections to the conductivity in the vortex-glass phase as in (8.12)-(8.14). Note that, at $T_{G}$, both the real and imaginary parts of the conductivity diverge as $\omega^{-(z+2-D) / z}$ as in Eq. (4.12), which again correctly matches the mean-field result at $D=6, z=4$. The low-frequency phase angle again should scale as in (4.13) and (4.14) but with different exponents $z$ and $v$. For example, for $z \simeq 5$, as estimated by Koch et al. ${ }^{22}$ the critical phase angle ${ }^{41}$ should be $\phi_{\sigma} \simeq 2 \pi / 5$.

In this section we have ignored the possible screening effects of an applied current that were discussed in Sec. VIII for the vortex-glass phase. In the vortex-fluid phase, the dc conductivity is finite and the penetration length in the limit of low frequencies is thus infinite so that a welldefined uniform dc conductivity exists. However, as the vortex-glass phase transition is approached, $\sigma(\omega)$ diverges for low frequencies. At the characteristic frequency $\Omega_{G} \sim \xi_{G}{ }^{z}$, the ac penetration length, assuming a uniform system, varies as

$$
\lambda_{G}\left(\omega=\Omega_{G}\right) \sim\left[\Omega_{G} \sigma\left(\Omega_{G}\right)\right]^{-1 / 2} \sim \xi_{G}^{(D-2) / 2} .
$$

This becomes very large near $T_{G}$, but grows less rapidly than the correlation length $\xi_{G}$ in three dimensions. When $\lambda_{G}\left(\Omega_{G}\right)$ becomes comparable to $\xi_{G}$, which sets the scale of the inhomogeneities in the local conductivity, it is certainly no longer appropriate to treat the system as uniform.

In this asymptotic regime, one must explicitly consider the effects of inhomogeneity on the transport properties. Whether this changes the scaling forms or the exponents for measurable quantities, or even destabilizes the ordered phase, eliminating the transition, we leave as an open question. The behavior in this regime is somewhat related to the asymptotic zero-field critical regime in which $\lambda \simeq \xi$ (see Sec. IV). In any case, if the field is high enough and the disorder strong enough that both $a_{v}$ and $L_{P}$ are much smaller than $\lambda\left(T_{G}\right)$, there will be a wide regime in which these effects can be ignored. Below $T_{G}$, however, the effect of finite current penetration on both linear and nonlinear transport measurements must be taken into account in large samples.

(3) Behavior as $T_{G} \rightarrow T_{c}$. In the $X Y$ critical regime at small fields and close to $T_{c}$, the vortex-glass transition field $B_{G}$ and the width of the vortex-glass critical region will (up to logarithms) both scale with the zero-field coherence length as $B_{G} \sim \xi^{-2}$ as does the melting line in the absence of disorder (5.10): thus,

$$
\left[T_{c}-T_{G}(H)\right] \sim H^{1 / 2 v_{0}},
$$

with $v_{0} \simeq \frac{2}{3}$ the zero-field exponent. ${ }^{55}$

(4) Small fields. For fields much less than $H_{X}$, the dynamics of a 3D vortex glass (and a reentrant vortexglass-to-liquid transition, which is possible at very low fields, $H \rightarrow H_{c 1}$, if the disorder is weak enough) may both be affected by whether or not vortex lines can cross and recombine in the time scale probed by a measurement. This relates to questions of vortex entanglement as studied by Nelson and Seung. 6,7 When the vortex separation $a_{v} \gg \xi$, there can be a large barrier for vortex recombination. If these processes are too slow to occur, the dynamic universality class of both the vortex-glass phase and the transition may well change. We leave these subtle and interesting, but currently probably not experimentally relevant, questions for future work.

\section{B. Theoretical evidence for the transition}

So far, we have not produced any evidence that a vortex-glass phase actually occurs in the 3D systems of interest. Indeed, since the broken symmetry is $X Y$-like and it appears ${ }^{13,56}$ that a conventional $X Y$ spin-glass phase does not exist for $T>0$ with short-range interactions in $3 \mathrm{D}$, the natural guess would be that the vortexglass phase is also unstable to thermal fluctuations in 3D.

Nevertheless, although this is largely an open question, we mention two suggestive pieces of evidence for the existence of a stable 3D vortex-glass phase at low temperatures. The first (already discussed by one of us in Ref. 11) is the existence of such a phase in two dimensions for a system of vortex lines. Although this represents a different dimensional continuation than the traditional one which preserves the $X Y$ symmetry but not the $1 \mathrm{D}$ character of the vortex lines, it is nevertheless highly suggestive. Indeed, even a single vortex line in a random potential is in a "glassy" phase ${ }^{50}$ with nontrivial response as discussed above in Sec. VIII. We also note that, if the disorder does not depend on the $z$ direction (direction of 
applied $H$ ), then the system is isomorphic to a 2D system of interacting bosons ${ }^{6,15}$ in a random potential at $T=0$, a system that has a (localized) glass phase in any dimension. 57

Another consideration is the nature of the symmetries of various 3D systems: Ising spin glass, $X Y$ spin glass, $X Y$ gauge glass (which is a lattice Landau-Ginzburg model with a quenched statistically isotropic random gauge field $\left.{ }^{58}\right)$, and the anisotropic vortex glass of interest with a dynamic gauge field. At first glance, the Ising system is radically different from the others in having only a discrete rather than a continuous symmetry. Nevertheless, as first pointed out by Villain, ${ }^{59}$ the continuous degrees of freedom in an $X Y$ spin glass can be integrated out in favor of discrete vortex variables which interact via long-range couplings. Thus, it must be some property of the interactions among the discrete degrees of freedom, rather than the spin-wave fluctuations, which destroys the $X Y$ spin-glass phase in 3D (assuming that it is indeed unstable at any $T>0$ ). The gauge glass and the vortex glass both have lower symmetries than the $X Y$ spin glass since they lack the discrete $\psi \rightarrow \psi^{*}$ symmetry (improper rotation in spin space or time-reversal invariance). Perhaps, these systems with lower orderparameter symmetries have a stronger tendency to order (see below). Here, again, the continuous degrees of freedom can be integrated out in favor of discrete vortex degrees of freedom ${ }^{15}$ - in these cases with no obvious remaining symmetries. In the case of a vortex glass with a fluctuating gauge field, the resulting interactions between the vortices are short ranged. Whether or not this appreciably distinguishes it from the extreme type-II case with a static $B$ field and infinite-ranged vortex interactions remains unclear at this point. Note, however, that both with and without a fluctuating gauge field the vortex glass is anisotropic due to the external field, in contrast to the other models. The observation above on the case with $z$-independent disorder suggests that anisotropy may tend to enhance ordering.

Numerical simulations ${ }^{31}$ of a 3D isotropic gauge glass with quenched random magnetic field show, perhaps surprisingly, that it is behaving more like the Ising than the $X Y$ spin glass and thus may well order at a nonzero $T_{G}$. A more convincing theoretical resolution of this important question may have to wait for high-quality Monte Carlo or high-temperature series work on simple models of the vortex glass. In the mean time, as we discuss in the final section of this paper, the experimental evidence in favor of a vortex-glass transition 22,60 - at least when the magnetic fluctuations can be ignored-is starting to mount up.

If a vortex-glass transition does not occur in $3 \mathrm{D}$ at positive temperature, then we expect the low-temperature behavior will be controlled by a zero-temperature vortex-glass transition with a glass correlation length $\xi_{G} \sim 1 / T^{v_{3}}$. This will result in a characteristic time scale and linear conductivity both diverging for $T \rightarrow 0$ as

$$
\tau \sim \exp \left(\Delta \xi_{G}^{\psi} / T\right) \sim \exp \left(b T^{-1-\psi v_{3}}\right),
$$

with the barrier exponent $\psi \leq 1$ and $b$ a constant. The current density where the crossover from Ohmic to nonlinear $I-V$ behavior occurs should, in this event, vanish for $T \rightarrow 0$ as

$$
J_{X} \sim T /\left(\xi_{G}^{2}\right) \sim T^{\left(1+2 v_{3}\right)},
$$

in contrast to the $J_{X} \sim T$ at low temperatures in the usual flux-creep theory. ${ }^{3,4}$ In this case, much of the lowtemperature behavior will be qualitatively similar to that discussed in the next Section.

\section{2D VORTEX-GLASS REGIMES}

In the absence of Josephson coupling between the layers, a layered superconductor can still undergo a superconducting transition in zero field to a phase which is a superconductor in the planes. This is true despite the fact that a "single-layer" sample will, strictly speaking, not have a positive-temperature superconducting phase due to out-of-plane magnetic fields which screen out the logarithmic interactions between vortices beyond a length $\lambda_{2 \mathrm{D}} \approx 2 \lambda^{2} / d$, with $d$ the layer thickness. (For thin-film "superconductors," $\lambda_{2 \mathrm{D}}$ is often macroscopic, ${ }^{61}$ so, in practice, one can have a good superconductor.) This out-of-plane screening effect will not happen in a system of stacked layers with no Josephson coupling; instead, the vortices in one layer will interact logarithmically at all distances.

For temperatures below the 2D Kosterlitz-Thouless transition $T_{\mathrm{KT}}$, the linear resistivity of such a system will vanish. However, as in the 3D Meissner phase, there will be nonlinear dissipation due to vortex nucleation: in this case vortex-antivortex pairs. Since the energy of a vortex pair grows logarithmically with separation, this gives rise to nucleation barriers which grow logarithmically with the current density and thus to power-law $I-V$ curves $^{19}$

$$
E \sim J^{\delta(T)}
$$

with $\delta(T)$ decreasing with increasing temperature and taking on the value 3 at $T_{\mathrm{KT}}$ (it jumps to unity for $\left.T>T_{\mathrm{KT}}\right)^{19}$

In a magnetic field above $H_{c 1}$, the field-induced vortices will destroy the 2D Kosterlitz-Thouless behavioralthough $2 \mathrm{D}$ vortex lattices are possible in a clean system. ${ }^{44}$ In the presence of disorder, a layered system can behave, over some range of temperature, as a stack of almost independent $2 \mathrm{D}$ vortex glasses. In strictly $2 \mathrm{D}$ superconductors, however, there is no positive-temperature vortex-glass phase. Rather, there will be a glass correlation length $\xi_{G}$ which diverges only at zero temperature as $\xi_{G} \sim T^{-v_{2}}$. The vortex-glass phase thus only truly exists at $T=0$ when the vortex positions are completely frozen. (Note, we neglect the interesting issue of quantum fluctuations of the vortices. ${ }^{62}$ ) On scales smaller than $\xi_{G}$, there will be barriers for vortex motion which may again grow logarithmically (they can grow no faster than $\ln \xi_{G}$ ) giving rise to a range of nonuniversal power-law $I-V$ curves. For small currents, however, the scales probed will be longer than $\xi_{G}$ and the system will act like a vortex fluid with finite Ohmic conductivity. In 2D, the characteristic length scale $L_{J}$, probed by a nonlinear 
current density $J$, is $L_{J} \sim c T /\left(J \phi_{0} d\right)$. Thus, the currènt density where the crossover from Ohmic to nonlinear behavior occurs should vanish as $J_{X} \sim\left(T / \xi_{G}\right) \sim T^{\left(1+v_{2}\right)}$ for $T \rightarrow 0$, or, depending on subtleties about how the current lowers the barriers, even more rapidly with temperature. This contrasts to the usual collective flux-creep picture where $^{3.4} J_{X} \sim T$. In practice, $J_{X}$ can be taken as the current density at which the ratio $E(J) / J$ is, say, twice the value of the linear $(J \rightarrow 0)$ resistivity. At low temperatures, there will also be a larger characteristic current density scale $J_{F}$ (as in an ordered phase) at which the onset of nonactivated vortex flow occurs.

If the barriers do grow logarithmically with $\xi_{G}$, this will give rise to a slightly faster than Arrhenius temperature dependence of the (linear) conductivity

$$
\sigma(T) \sim e^{(b|\ln T|) / T} .
$$

At low enough temperatures, however, the magnetic interactions between vortices on different layers of a layered system or the residual Josephson coupling between layers will yield a crossover to 3D vortex-glass behavior. How this crossover occurs in various regimes we leave for future investigations.

\section{EXPERIMENTS}

In this section we discuss several pertinent experiments. We consider first experiments on $\mathrm{YBa}_{2} \mathrm{Cu}_{3} \mathrm{O}_{7}$ (Y-Ba-Cu-O) - the best studied high- $T_{c}$ material with regard to macroscopic electromagnetic properties.

\section{A. Y-Ba-Cu-O}

Before discussing transport and related measurements, we comment on the relevant experimental parameters. The zero-field transition temperature $T_{c}$ for $\mathrm{Y}-\mathrm{Ba}-\mathrm{Cu}-\mathrm{O}$ is typically near $90 \mathrm{~K}$. A series of low-temperature penetration length measurements by a variety of techniques ${ }^{63}$ have found the in-plane penetration length at low temperature to be $\lambda_{1}(T=0) \simeq 1400 \AA$. The temperature dependence of $\lambda_{\perp}$ up to $\simeq \frac{1}{2} T_{c}$ is less than for conventional superconductors suggesting that the amplitude $\lambda_{10}$ of the penetration length

$$
\lambda_{\perp}(T) \approx \lambda_{\perp 0}\left(\frac{T_{c}-T}{T_{c}}\right)^{1 / 2}
$$

in the Ginzburg-Landau regime may be rather smaller than, say, the conventional dirty limit result $\lambda_{10} \simeq 0.6 \lambda(0)$, so that we expect $\lambda_{10} \lesssim 800 \AA$. A penetration length anisotropy parameter $\gamma=\lambda_{1} / \lambda_{z} \simeq 0.2$ has been found at low temperatures. ${ }^{64}$ Unfortunately, there is no good measurement of the coherence length. Below $T_{c}$ in the presence of fluctuations it is, in fact, poorly defined. The appropriate $\xi$ from above $T_{c}$, which is conventionally used in the Ginzburg-Landau theory and remains well defined with fluctuations, cannot be directly measured. Thus, there is considerable uncertainty in the Ginzburg-Landau $\xi_{10}$ and hence also $\kappa$. Assuming a $\kappa \simeq 100$, the width of the critical region as given by Eq. (4.8) is $\varepsilon_{x} \simeq 0.1$, and thus the conventional Ginzburg criterion [described just below Eq. (4.8)] would yield $\varepsilon_{x} \simeq 4 \times 10^{-3}$ for specificheat experiments roughly consistent with the data. ${ }^{65}$ In any case, much of the phase diagram of $\mathrm{Y}-\mathrm{Ba}-\mathrm{Cu}-\mathrm{O}$ will be in the Ginzburg-Landau regime and the anisotropy is weak enough that two-dimensional effects will not play a large role.

There are two natural sources of small-scale disorder in Y-Ba-Cu-O; oxygen vacancies or interstitials, for which the estimates in Sec. VI should apply, and semimicroscopic twin boundaries which might appear in epitaxially grown films. Provided the spacing between twin boundaries is less than the vortex lattice constant $a_{v}=\left(\phi_{0} / B\right)^{1 / 2}$ and they are randomly located, the qualitative effects will be similar to those of vacancies, although correlations between the locations of the defects in successive planes may lead to some differences. We first consider the apparent phase boundary for onset of high conductivity as the temperature is lowered in a strong magnetic field $\gg H_{c 1}\left(H_{c 1} \simeq 200 \mathrm{G}\right.$ at low $\left.T\right)$.

Traditionally, dc magnetic susceptibility measurements are used to infer $H_{c 2}$. However, in the presence of fluctuations, the mean-field transition $H_{c 2}^{\mathrm{MF}}(T)$ is just a crossover line, near which the resistivity will begin to drop, and a significant diamagnetism will develop. ac susceptibility measurements ${ }^{21}$ on single-crystal $\mathrm{Y}-\mathrm{Ba}-\mathrm{Cu}-\mathrm{O}$ have revealed significant structure at temperatures well below this crossover line, at least for strong fields $(H \geq 10 \mathrm{kG})$. Specifically, the response to an additional small ac field at frequency $\omega$, applied parallel to the dc field, was found to exhibit a sharp diamagnetic signature in $\operatorname{Re} \chi(\omega)$ and a corresponding peak in $\operatorname{Im} \chi(\omega)$ at a fairly well-defined "irreversibility" temperature $T_{\text {irr }}(\omega)$. The resulting irreversibility line plotted in the $H-T$ plane lies well below the conventionally estimated $H_{c 2}^{\mathrm{MF}}$ line and shows significant frequency dependence, particularly at high fields. ${ }^{21}$

These ac susceptibility measurements are essentially an indirect measure of the resistivity of a sample. For $T>T_{\text {irr }}$ the vortex-line mobility, which is proportional to the sample resistivity, is sufficiently large to allow additional vortex lines to diffuse fully into and out of the sample (or the region of the perturbing field) in a period of the ac field. For $T \lesssim T_{\text {irr }}, \operatorname{Re} \chi(\omega)$ become more diamagnetic since additional vortex lines diffuse too slowly to appreciably penetrate the sample and dissipate the screening currents. Detailed interpretation of these measurements, though, depends on the experimental geometry.

The regime below the crossover $H_{c 2}^{\mathrm{MF}}$ line, but above the irreversibility lines, can be conveniently described as a vortex liquid ${ }^{18}$ (except perhaps at low fields for $T \gtrsim T_{c}$ in the $X Y$ critical regime). Conventional flux-creep theory ${ }^{3,4}$ would predict that, when probed at sufficiently low frequencies, this vortex-liquid regime would persist down to $T=0$, i.e., $T_{\text {irr }}(\omega) \rightarrow 0$ in the $\omega \rightarrow 0$ limit. In contrast, if there is a low-temperature vortex-glass phase, $T_{\text {irr }}(\omega)$ will approach the glass transition temperature $T_{G}$ in the limit $\omega \rightarrow 0$. More specifically, scaling of the conductivity as in Eq. (4.11) implies that

$$
\left[T_{\mathrm{irr}}(\omega)-T_{g}\right] \sim \omega^{1 /[(z+2-D) v]}
$$

for small $\omega$. (Note that this scaling is not $\Delta T \sim \omega^{1 / z v}$ because of the geometry effects.)

So far, the only direct experimental probe of the vortex correlations that has been used is imaging of the flux lines 
after Bitter decoration with magnetic particles. Initial bitter decorations on field-cooled single-crystal Y-Ba-CuO samples showed only short-range $(\sim 2-3$ lattice spacings) order. ${ }^{20}$ As with all Bitter decorations, one is restricted to low fields (in this case, 10-200 G) where the vortex-line separation is larger than the penetration length, so that the magnetic flux tubes on adjacent vortices do not overlap too much. Later experiments on different crystals revealed longer-ranged order in twinfree regions of the samples, suggesting twin boundaries were responsible for the disorder in the earlier results. ${ }^{20}$ In the latter crystals, the vortex-lattice was surprisingly immobile at temperature $T \simeq 4 \mathrm{~K}$ when the field strength was varied or turned off. Dolan et al. ${ }^{20}$ attributed this, very plausibly, to microscopic pinning defects such as oxygen vacancies.

In a film geometry, as used by Dolan et al. ${ }^{20} B \simeq H$ which is fixed while the sample is cooled. As can be seen From Fig. 4, the disorder-free melting transition is reentrant at fixed small $B$. As the system is cooled, it will try to form a lattice at the high-field melting transition which will be in the $X Y$ critical regime. Here, the effects of microscopic disorder can be weak, since large thermal fluctuations of the vortex lines are effective at averaging it out, and a relatively long lattice correlation length $\sim a_{v} / \Delta_{D}^{\mathrm{NN}}$ as estimated by (6.8) for microscopic pinning may occur. As the temperature is decreased, the effects of the disorder increase as shown in (6.8). However, once the lattice is formed, the vortex lines cannot easily rearrange. Thus, if the sample is cooled at a reasonable rate, the vortex lines will fall out of equilibrium, with the microscopic pinning effectively freezing in position the quite long-range lattice order which was established near $T_{c}$. The apparent long-range-ordered lattice phase, as seen in the Bitter decorations on untwinned samples, would then be a metastable state. The true equilibrium lowtemperature phase, as originally argued by Larkin and Ovchinikov, ${ }^{9}$ does not have long-ranged crystalline order and may well be a vortex glass (see below).

For fields low enough that a reentrant fluid appears, the eventual freezing transition will take place at lower temperatures (see Fig. 4) where the effects of disorder will be much larger, as shown in (6.8); the lattice correlations will thus be of much shorter range. Preliminary evidence for this has been seen by Dolan et al. ${ }^{20}$ at very low fields.

We now turn to much higher fields, $H \gtrsim 5 \mathrm{kG}$, where most of the transport measurements have been carried out. If the disorder were indeed unimportant near the pure system's vortex-liquid to lattice freezing temperature and this transition were first order as expected, ${ }^{47}$ one would not find significant precursor effects in properties such as transport above this temperature. However, the irreversibility lines measured by ac susceptibility (as described above) can vary appreciably with frequency, particularly at high fields and frequencies [e.g., at $50 \mathrm{kG}$, $T_{\text {irr }}(\omega)$ drops by $\simeq 3 \mathrm{~K}$ when $\omega$ is reduced from 3.0 to 0.1 $\mathrm{MHz}$ (Ref. 66)]. Disorder is thus apparently playing a far more significant role here at high fields than in the lowfield Bitter decorations. This is entirely consistent with the field dependence in (6.8), which shows the increasing effect of microscopic disorder with increasing field near the melting transition and outside of the $X Y$ critical regime. In addition to the field dependence, the crystals used in the susceptibility and most of the transport measurements are believed to have a higher twinning density than in the "best" decoration samples. Thus, pinning from twins may further increase the importance of disorder in these samples.

Neither of the experiments described above shed significant light on the fundamental question of whether or not a true equilibrium superconducting phase is present at low temperature for fields above $H_{c 1}$; i.e., in the presence of pinning is a zero-resistance vortex-glass phase present or is there always a small, but nonzero, linear resistance as in conventional flux-creep theory? Recent nonlinear current-voltage $(I-V)$ measurements on $0.4-\mu \mathrm{m}$-thick epitaxial films of $\mathrm{Y}-\mathrm{Ba}-\mathrm{Cu}-\mathrm{O}$ by Koch et al. ${ }^{22}$ offer preliminary evidence of a vortex-glass phase. As described in Secs. IV C and IX, the presence of a continuous normal-to-superconducting phase transition should leave a distinctive signature in the nonlinear $I-V$ characteristics. At the transition temperature $T_{G}$, a power-law $I-V$ is expected at low current densities, as in (4.19) and (9.2). For $T$ near $T_{G}$, the $I-V$ characteristics should approach this power law for currents bigger than $J_{X \perp}$ in (4.15), crossing over to Ohmic behavior at low current for $T>T_{G}$, and to an exponentially vanishing voltage for $T<T_{G}$. Precisely such behavior was found by Koch et al. ${ }^{22}$ with a voltage appearing to vanish more rapidly than a power of $J$ for $T<T_{G}$. The $I-V$ data were found to scale as predicted by (4.18) and (9.1) near $T_{G}$, and critical exponents $z$ and $v$ were extracted. The values obtained for the higher-field data $(H=20-40 \mathrm{kG}), z \simeq 5$ and $v \simeq 1.8$, are consistent with expectations for the vortex-liquid-to-glass transition (Sec. IX). Note that the absence of any discontinuities in the low current data appears to rule out all but a very weak first-order transition.

Very recent data $^{67}$ on thin films, 250-500- $\AA$ thick, show that the signature of the transition in the $I-V$ characteristic is washed out, consistent with theoretical expectations that a $T \neq 0$ vortex-glass phase can only exist in bulk (3d) samples.

It is instructive to examine the length scales probed in the Koch et al. ${ }^{22}$ measurements. At current density $J$, one is effectively probing the system on a length scale

$$
L_{J}=\left(c k_{B} T / J \phi_{0}\right)^{1 / 2},
$$

as seen from Eq. (4.18). (More correctly, because of the intrinsic anisotropy and that due to the field, $L_{J}$ is roughly the geometric mean of two lengths, parallel and perpendicular to the field, whose ratio is likely to be of order $\gamma$.) For $H=40 \mathrm{kG}$, the range of current densities measured at $T_{G}$ corresponds to lengths from about 200 to $5000 \AA$. (Thus, suggesting why the transition is washed out for $250-500-\AA$ thick films. ${ }^{67}$ ) Deviations from the critical power law are not evident, even at the highest current densities, indicating that the vortex-liquid to glass critical regime is very wide, extending down to length scales comparable to the intervortex spacing $(\sim 200 \AA$ at $40 \mathrm{kG})$. At $H=5 \mathrm{kG}$, deviations from 
power-law behavior are seen at currents corresponding to a length of about $600 \AA$, roughly equal to the intervortex separation. This feature of the data suggests that, in these films, disorder is playing a strongly dominant role, destroying the vortex-lattice translational order on a scale of a few lattice spacings.

Very recent nonlinear $I-V$ data on single crystals ${ }^{60}$ show features which are not inconsistent with a vortexglass transition, but with a critical regime which is $m u c h$ narrower than in the films studied by Koch et al. ${ }^{22}$ At $H=70 \mathrm{kG}$, the $I-V$ curve at the apparent transition temperature shows only a narrow regime of low currents which could be consistent with the expected power law. This occurs only for currents corresponding to lengths $L_{J}$ greater than about a micron, or roughly 75 times the intervortex spacing. This length may thus correspond to the crossover length $L_{P}$ from lattice to vortex-glass critical behavior as discussed in Sec. VII - although the data may also be consistent with a weakly first-order transition. Further study of such very clean samples will be instructive.

It is possible that the differences between the $I-V$ data on films and crystals are due to very different twinning densities. The crystals are believed to have twins on the micron scale, whereas, due to the epitaxial growth, the films might possibly have twins with spacing of order 100 $\AA$ which would effectively act like microscopic pinning. Clearly more work is needed here. (Note, a recent paper by Marchetti and Nelson ${ }^{68}$ has interpreted the data in the fluid phase of Ref. 60 in terms of widely spaced strong pinning sites impeding the vortex flow.)

Another important experiment which can, in principle, distinguish between a vortex-glass phase and AndersonKim "flux creep" is the decay of a remanent (persistent) current. In the vortex-glass phase a persistent current is predicted to decay as ${ }^{30}$

$$
J(t) \approx J_{F}\left[1+(T / U) \ln \left(t / t_{0}\right)\right]^{-1 / \mu},
$$

from (8.7), with $U$ roughly temperature independent for $T$ below $\simeq 0.5 T_{G}$. This form implies that, for a fixed range of $\ln t,-\partial J / \partial \ln t$ is a non-monotonic function of temperature, decreasing as $T^{-1 / \mu}$ above a crossover temperature $T_{\mathrm{cr}} \approx U / \ln \left(t / t_{0}\right)$. This is in contrast to the Anderson-Kim theory which predicts an exponential decay of $J(t)$ for temperatures above $T_{\mathrm{cr}}$. Magnetic relaxation data by Yeshurun et al. ${ }^{51}$ on $\mathrm{Y}-\mathrm{Ba}-\mathrm{Cu}-\mathrm{O}$ crystals are consistent with a logarithmic time decay over the range from 200 to $4000 \mathrm{sec}$, measured for all temperatures below $80 \mathrm{~K}$ in a $600-\mathrm{G}$ field. Moreover, a striking nonmonotonicity is seen in $-\partial J / \partial \ln t$ with a peak at a crossover temperature $\sim 20 \mathrm{~K}$. The data are consistent with the form predicted in the vortex-glass phase, (8.7), with a value of $U$ in the range $200-300 \mathrm{~K}$. Further relaxation experiments on other materials and over a wider range in time and sample size are clearly desirable, especially in order to distinguish between this interpretation and the more conventional one given by Yeshurun et al. ${ }^{51}$

An important measurement that has not yet been done is to measure the frequency-dependent linear conductivity over a wide frequency range near the vortex-glass tran- sition to compare to the scaling forms proposed in Sec. IV B. Evidence for a divergent time scale in the linear conductivity which diverges at the same temperature as the nonlinear data indicates the phase transition would provide extra evidence for a true vortex-glass transition. In particular, it would help to rule out effects due to macroscopic inhomogeneities which, similar to Sec. VIII, affect the linear and nonlinear conductivity in rather different ways.

The magnetic field scale of the vortex-glass transition found by Koch et al. ${ }^{22}$ is roughly consistent with being a small fraction of $H_{X}$, i.e., $B_{G} \approx c_{G} \phi_{0} / 2 \pi \xi_{\perp}^{2}$ with $c_{G}$ of or$\operatorname{der} 10^{-1}$ if we use $\xi_{10}=10 \AA$. In the $X Y$ critical region, this is consistent with the glass transition occurring near to where the lattice melting transition would be in the absence of disorder. In the region near to $T_{c}$ we expect that $\left(T_{G}-T_{c}\right) \sim H^{1 / 2 v}$, with $v \simeq \frac{2}{3}$ due to critical fluctuations. Some curvature of the phase boundary is indeed seen in the Koch et al. ${ }^{22}$ data consistent with this behavior. The phase boundary (presumably separating vortex-glass and liquid phases) found by Worthington et al. ${ }^{60}$ is also consistent with a $\left(T_{G}-T_{c}\right) \sim H^{1 / 2 v}$ dependence, as are earlier experiments which measure various other crossover lines. ${ }^{69}$

It is important to note that the data so far, while strongly suggestive of a vortex-glass transition, have not probed the regime in which the magnetic fluctuations become important, which occurs when $\lambda_{G} \sim \xi_{G}$ (Sec. IX). Thus, a rounding of the liquid-to-glass transition and a destruction of the vortex-glass phase itself by magnetic fluctuations has not been ruled out, although there is no particular reason to expect that this will occur.

\section{B. Bi-Sr-Ca-Cu-O}

We now turn to experiments on $\mathrm{Bi}_{2} \mathrm{Sr}_{2} \mathrm{CaCu}_{2} \mathrm{O}_{8}$ (Bi-Sr-Ca-Cu-O) which shows strikingly different resistive behavior in magnetic fields. Indeed, in modest fields of a few tens of kilogauss, $\mathrm{Bi}-\mathrm{Sr}-\mathrm{Ca}-\mathrm{Cu}-\mathrm{O}$ does not become a better conductor than good copper until the temperature is below $30 \mathrm{~K},{ }^{23}$ a factor of 3 below the zero-field transition at $T_{c} \simeq 85 \mathrm{~K}$.

We first estimate the relevant Ginzburg-Landau parameters. $\mathrm{Bi}-\mathrm{Sr}-\mathrm{Ca}-\mathrm{Cu}-\mathrm{O}$ is far more anisotropic than $\mathrm{Y}-\mathrm{Ba}-\mathrm{Cu}-\mathrm{O}$ with an apparent anisotropy ${ }^{29}$ of $\gamma=\lambda_{\perp} / \lambda_{z} \lesssim \frac{1}{50}$. We use $\gamma \simeq \frac{1}{50}$ in the estimates below. Direct measurements of the in-plane penetration length have not been performed, and preliminary indirect evidence from muon-spin resonance, ${ }^{70}$ which measures the root-mean-square variations in the local magnetic field that are proportional to $1 / \lambda^{2}$ for a weakly fluctuating vortex lattice in which the muon does not significantly perturb the vortex locations, would yield the surprising conclusion that, at $\sim 20 \mathrm{~K}, \lambda_{\perp} \sim 5000 \mathrm{~A}$. We will see below that an alternative interpretation of this data is perhaps somewhat more plausible.

Another indirect measurement of $\lambda_{\perp}$ near $T_{c}$ has been made by Martin et al. ${ }^{71}$ who observe that the linear resistivity just above $T_{c}$ in zero field fits well a twodimensional Kosterlitz-Thouless form for uncoupled lay- 
ers. This is eminently reasonable since the in-plane correlations must grow to distances $\sim \xi^{\mathrm{MF}}\left(T_{c}\right) / \gamma$ before the interplane Josephson coupling causes three-dimensional order. From this data, ${ }^{71}$ and the universal relationship between $\lambda\left(T_{c}\right)$, the layer thickness $d \simeq 15 \AA$, and $T_{c}$ in two dimensions, one extracts $\lambda\left(T_{c}\right) \simeq 4000 \AA$. From a fit to the Aslamasov-Larkin fluctuation resistivity at higher temperatures, Martin et al. ${ }^{71}$ find a $2-\mathrm{K}$ suppression of the true transition $T_{c}$ from the mean-field transition $T_{\text {BCS }}$, essentially the width of the resistive transition. This suggests that the Ginzburg-Landau critical amplitude is $\lambda_{10} \sim 600 \AA$, not inconsistent with $\mathrm{Y}-\mathrm{Ba}-\mathrm{Cu}-\mathrm{O}$, but hardly consistent with the naive interpretation of the muon-spin resonance data mentioned above. Note that a $\lambda_{10}$ of several thousand $\AA$ would have given rise to an extremely broad resistive transition in zero field, so is quite inconsistent with the resistivity data.

Although the zero-field resistive transition in $\mathrm{Bi}-\mathrm{Sr}-\mathrm{Ca}-\mathrm{Cu}-\mathrm{O}$ is rather sharp (comparable to $\mathrm{Y}-\mathrm{Ba}-\mathrm{Cu}-$ $\mathrm{O})$, the behavior in a field is quite different. Palstra et $a l .{ }^{23}$ have found that, in fields above a few $\mathrm{kG}$, the linear resistivity appears approximately Arrhenius over a wide temperature range, down to below $20 \mathrm{~K}$ at $100 \mathrm{kG}$ with activation energies decreasing from $\sim 1000 \mathrm{~K}$ at 1 $\mathrm{kG}$ to $\sim 300 \mathrm{~K}$ at $100 \mathrm{kG}$.

Frequency-dependent susceptibility data reported by Gammel $^{24}$ yield the temperature dependence of a relaxational frequency of the vortices which is again close to Arrhenius with similar field-dependent barriers to those found in the resistive data ${ }^{23}$ although deviations from Arrhenius law are seen. At the lowest frequency of $10 \mathrm{~Hz}$, the vortices do not respond below about $26 \mathrm{~K}$ in $10 \mathrm{kG}$ and $19 \mathrm{~K}$ in $50 \mathrm{kG}$, while at the highest frequency of 10 $\mathrm{MHz}$, the temperatures are $\sim 70$ and $50 \mathrm{~K}$, respectively. The distribution of relaxation frequencies inferred from the ac susceptibility ${ }^{24}$ at a given field and temperature is rather broad, as expected for glassy behavior, although its dependence on the parameters has not yet been analyzed.

Given the weak Josephson coupling between the layers, which will be particularly ineffective at high fields (see Sec. V), a natural interpretation of these data is in terms of approximately independent sets of point vortices in each layer. These vortices will be strongly affected by disorder at low temperatures and thus tend to form a stack of weakly coupled $2 \mathrm{D}$ vortex glasses. As described in Sec. $X$, each individual layer has a transition only at zero temperature with a relaxational time diverging with an Arrhenius law or perhaps slightly faster as $\tau \sim e^{+b|\ln T| / T}$. The field dependence of the barrier is rather complicated. However, we expect that, as the field is lowered, the enhanced magnetic coupling between the layers and the increased local order parameter $\psi$ will tend to make the barriers grow, consistent with the experiments. ${ }^{20,21}$ We leave a detailed analysis for future work.

Evidence for a somewhat-faster-than-Arrhenius behavior for the characteristic time is found in the ac susceptibility data: $\mathrm{Gammel}^{24}$ fits $\tau \sim \exp \left(1 / T^{1.5}\right)$ [which could correspond to a 3D zero-temperature vortex-glass transition with $\psi v_{3}=\frac{1}{2}$ as in (9.5)], but the data also fits to a power-law divergent time at a positive-temperature 3D glass transition with $T_{G} \approx 14 \mathrm{~K}$ at $50 \mathrm{kG}$ increasing to $\sim 24 \mathrm{~K}$ at $5 \mathrm{kG}$. This latter behavior is what one would expect when the three-dimensional coupling between the layers comes into play, which it will do at higher temperatures in smaller fields. The crossover between two- and three-dimensional regimes at a fixed $\sim 3 \mathrm{kG}$ as suggested by Eqs. (5.5) and (5.8) is consistent with the data. ${ }^{24}$

A natural scale for the characteristic temperature in high fields (but $\ll H_{c 2}^{\mathrm{MF}}$ ) is given by estimating the $2 \mathrm{D}$ vortex-lattice melting temperature in the absence of disorder. For $T_{M}=15 \mathrm{~K}$, Eq. (5.5) yields $\lambda_{\perp}\left(T_{M}\right)$ $=1500-2000 \AA$, consistent with Y-Ba-Cu-O and the data of Martin et al. ${ }^{71}$ near $T_{c}$ if we assume a rather flat temperature dependence of $\lambda(T)$ at low temperatures. Note that the relatively large activation barriers indicated by the transparent data are evidence for quite strong correlations within each layer, since the core pinning energy of a single one-layer vortex is at most only of order $200 \mathrm{~K}$, even if a hole is punched in the film. Thus, we expect the characteristic temperatures to be determined by the correlation yielding $T_{G} \lesssim T_{M}$.

Further evidence for the role of vortex correlations could be obtained from nonlinear $I-V$ curves. As discussed in Sec. X, appreciable nonlinearities will be expected due to suppression of barriers when the length scale probed by the current density $L_{J} \sim\left(c k_{B} T\right) / J \phi_{0} d$ in two dimensions (with $J$ the $3 \mathrm{D}$ current density) becomes of order the (2D) glass correlation length $\xi_{G}$. Thus, at low temperatures, the characteristic current for nonlinearities $J_{X}$ scales as $T / \xi_{G}$ which vanishes as $T^{1+v_{2}}$ in the $2 \mathrm{D}$ regime. By contrast, traditional flux-creep mod$\mathrm{els}^{3-5}$ assume a temperature-independent characteristic length for vortex creep yielding $J_{X} \sim T$. Operationally, $J_{X}$ can be taken as the current density at which the ratio $E / J$ is, say, twice the value of the linear, Ohmic $(J \rightarrow 0)$ resistivity. Note that, at low temperatures, as in an ordered vortex-glass phase, there is another large characteristic current scale $J_{F}$ for onset of strong vortex flow. This will act as an approximately temperatureindependent apparent critical current at low temperatures.

At smaller fields, the interlayer couplings will play a more dominant role, increasing $T_{G}$, and causing a crossover to $3 \mathrm{D}$ behavior.

In very small fields $B \sim 50 \mathrm{G}$, Bitter decoration shows a well-correlated flux lattice in carefully prepared samples, ${ }^{24}$ as for $\mathrm{Y}-\mathrm{Ba}-\mathrm{Cu}-\mathrm{O}$. In such small fields the magnetic interlayer coupling is dominant, so that the behavior is three dimensional and the differences between $\mathrm{Y}-\mathrm{Ba}-\mathrm{Cu}-\mathrm{O}$ and $\mathrm{Bi}-\mathrm{Sr}-\mathrm{Ca}-\mathrm{Cu}-\mathrm{O}$ should be much less pronounced.

This leaves us with the puzzle of understanding the muon-spin resonance ( $\mu \mathrm{SR})$ data on $\mathrm{Bi}-\mathrm{Sr}-\mathrm{Ca}-\mathrm{Cu}-\mathrm{O} .{ }^{70}$ What is effectively measured here is the inhomogeneity in the local fields (in a fixed applied field $\leq 4 \mathrm{kG}$ ) averaged over the lifetime $\tau_{\mu} \simeq 2 \times 10^{-6}$ sec of the muons. Several effects can decrease the measured inhomogeneity $\Delta B$. Disorder, which causes the vortices in each layer to be pinned independently of those in other layers, gives rise to some suppressions [by a factor $\sim\left(d / a_{v}\right)^{1 / 2}$ ] as does 
motional averaging due to vortex motion on time scales $\lesssim \tau_{\mu}$. However, thermal vibrations of vortex lines by $\sim a_{v}$ about quasiequilibrium positions will only suppress $\Delta B$ by relatively small amounts. Thus, neither of these effects appear to be sufficient to reduce $\Delta B$ by the factor $\geq 20$ found in the experiments at temperatures $\geq 15 \mathrm{~K}$. Another effect, which certainly appears to occur, ${ }^{70}$ is local distortions of the vortex positions around the charged muon. Because of its quasi-two-dimensionality, these distortions are expected to be much larger in Bi-Sr-Ca-Cu-O than Y-Bi-Cu-O and complicate the analysis of the $\mu \mathrm{SR}$ data for $\mathrm{Bi}-\mathrm{Sr}-\mathrm{Ca}-\mathrm{Cu}-\mathrm{O}$. Thus, we leave as a puzzle the apparent discrepancies between the estimates of $\lambda$, which may well be due to large differences between the types of samples used.

\section{CONCLUSION}

We have seen that much of the data on $\mathrm{Y}-\mathrm{Ba}-\mathrm{Cu}-\mathrm{O}$ and $\mathrm{Bi}-\mathrm{Sr}-\mathrm{Ca}-\mathrm{Cu}-\mathrm{O}$ can be qualitatively and sometimes semiquantitatively explained in terms of the theoretical framework established here and in earlier papers. More experiments, in a variety of regimes of temperature, field, and anisotropy are clearly needed. It is especially important to make nonlinear transport and frequency-dependent linear susceptibility or conductivity measurements on the same sample since differences between samples appear to be potentially quite large. In addition, modifying the pinning in a controlled way would be especially instructive.

Finally, we note that many of the fluctuations effects which arise in high- $T_{c}$ superconductors also arise in other "weak" superconductors, such as films, granular superconductors, and artificially layered materials. Further investigations of these other types of materials, where parameters are better known and may be more easily controlled, are clearly called for.

\section{ACKNOWLEDGMENTS}

We thank G. Aeppli, D. J. Bishop, E. H. Brandt, E. Fradkin, P. L. Gammel, B. I. Halperin, A. F. Hebard, P. C. Hohenberg, R. H. Koch, P. B. Littlewood, A. P. Malozemoff, T. T. M. Palstra, S. H. Shenker, E. D. Siggia, A. Sudb $\varnothing$, J. Toner, and T. K. Worthington for useful discussions. One of us (D.S.F.) was partially supported by the National Science Foundation (NSF) under Grant No. DMR 8719523 and by the Alfred P. Sloan Foundation.
*Present address: Physics Department, Harvard University, Cambridge, MA 02138.

${ }^{1}$ For a review of the mean-field theory of type-II superconductors see, A. L. Fetter and P. C. Hohenberg, in Superconductivity, edited by R. D. Parks (Dekker, New York, 1969), Vol. 2.

${ }^{2}$ For a review of fluctuations about mean-field theory see, W. J. Skocpol and M. Tinkham, Rep. Prog. Phys. 38, 1049 (1975).

${ }^{3}$ P. W. Anderson and Y. B. Kim, Rev. Mod. Phys. 36, 39 (1964).

${ }^{4} \mathrm{~A}$ recent discussion of collective vortex pinning is $\mathrm{P}$. $\mathrm{H}$. Kes, $\mathrm{J}$. Aarts, J. van den Berg, C. J. van der Beek, and J. A. Mydosh, Supercond. Sci. Tech. 1, 242 (1989).

${ }^{5}$ M. Inui, P. B. Littlewood, and S. N. Coppersmith, Phys. Rev. Lett. 63, 2421 (1989).

${ }^{6}$ D. R. Nelson, Phys. Rev. Lett. 60, 1973 (1988); J. Stat. Phys. 57, 511 (1989).

${ }^{7}$ D. R. Nelson and H. S. Seung, Phys. Rev. B 39, 9153 (1989).

${ }^{8}$ See, e.g., Y. B. Kim and M. J. Stephen, in Superconductivity (Ref. 1).

${ }^{9}$ A. I. Larkin and Yu. N. Ovchinikov, J. Low Temp. Phys. 34, 409 (1979).

${ }^{10}$ W. Y. Shih, C. Ebner, and D. Stroud, Phys. Rev. B 30, 134 (1984).

${ }^{11}$ M. P. A. Fisher, Phys. Rev. Lett. 62, 1415 (1989).

${ }^{12}$ S. John and T. C. Lubensky, Phys. Rev. B 34, 4815 (1986).

${ }^{13}$ For a review of spin glasses see, K. Binder and A. P. Young, Rev. Mod. Phys. 58, 801 (1986).

${ }^{14}$ D. S. Fisher and D. A. Huse, Phys. Rev. B 38, 386 (1988); 38, 373 (1988).

${ }^{15}$ M. P. A. Fisher and D. H. Lee, Phys. Rev. B 39, 2756 (1989).

${ }^{16}$ A. Houghton, R. A. Pelcovits, and S. Sudbo, Phys. Rev. B 40, 6763 (1989).

${ }^{17}$ E. H. Brandt, Phys. Rev. Lett. 63, 1106 (1989).

${ }^{18}$ P. L. Gammel, L. F. Schneemeyer, J. V. Waszczak, and D. J. Bishop, Phys. Rev. Lett. 61, 1666 (1988).

${ }^{19}$ V. Ambegaokar, B. I. Halperin, D. R. Nelson, and E. D. Sig- gia, Phys. Rev. B 21, 1806 (1980).

${ }^{20}$ P. L. Gammel, D. J. Bishop, G. J. Dolan, J. R. Kwo, C. A. Murray, L. F. Schneemeyer, and J. V. Waszczak, Phys. Rev. Lett. 59, 2592 (1987); G. J. Dolan, G. V. Chandrashekhar, T. R. Dinger, C. Field, and F. Holtzberg, ibid. 62, 829 (1989).

${ }^{21}$ A. D. Malozemoff, T. K. Worthington, Y. Yeshurun, F. Holtzberg, and P. H. Kes, Phys. Rev. B 38, 7203 (1988).

${ }^{22}$ R. H. Koch, V. Foglietti, W. J. Gallagher, G. Koren, A. Gupta, and M. P. A. Fisher, Phys. Rev. Lett. 63, 1511 (1989). A very recent extension to this work to much (3 orders of magnitude) lower current densities and a crystalline sample is $\mathbf{P}$. L. Gammel, L. F. Schneemeyer, and D. J. Bishop (unpublished).

${ }^{23}$ T. T. M. Palstra, B. Batlogg, L. F. Schneemeyer, and J. V. Waszczak, Phys. Rev. Lett. 61, 1662 (1988).

${ }^{24}$ P. L. Gammel, J. Appl. Phys. 67, 4676 (1990).

${ }^{25}$ See, e.g., J. S. Langer and M. E. Fisher, Phys. Rev. Lett. 19, 560 (1967).

${ }^{26}$ B. I. Halperin, T. C. Lubensky, and S. K. Ma, Phys. Rev. Lett. 32, 292 (1974).

${ }^{27}$ C. Dasgupta and B. I. Halperin, Phys. Rev. Lett. 47, 1556 (1981).

${ }^{28}$ See S. A. Wolf, D. U. Gubser, and Y. Imry, Phys. Rev. Lett. 43, 324 (1979) for an early paper on scaling of the nonlinear conductivity in thin-film superconductors.

${ }^{29}$ D. E. Farrell, S. Bonham, J. Foster, V. C. Chang, P. Z. Jiang, K. G. Vandervoort, D. J. Lam, and V. G. Kogan, Phys. Rev. Lett. 63, 782 (1989).

${ }^{30}$ T. Nattermann, Phys. Rev. Lett. 64, 2454 (1990).

${ }^{31}$ D. A. Huse and H. S. Seung, Phys. Rev. B 42, 1059 (1990).

${ }^{32}$ The conventionally defined coherence length actually represents the exponential decay length of correlations at equivalent reduced temperature above $T_{c}$. With fluctuations, the coherence length is ill defined below $T_{c}$ and we thus use this convention throughout the discussion of the small- and zero-field critical behavior, particularly in Sec. IV. 
${ }^{33}$ For analogous effects in Ising systems see D. A. Huse and D. S. Fisher, Phys. Rev. B 35, 6841 (1987).

${ }^{34}$ A. Singsaas and G. Ahlers, Phys. Rev. B 30, 5103 (1984).

${ }^{35}$ B. I. Halperin and D. R. Nelson, J. Low Temp. Phys. 36, 599 (1979).

${ }^{36}$ M. E. Fisher, M. N. Barber, and D. Jasnow, Phys. Rev. A 8, 1111 (1973).

${ }^{37}$ P. C. Hohenberg, A. Aharony, B. I. Halperin, and E. D. Siggia, Phys. Rev. B 13, 2986 (1976).

${ }^{38}$ For a review of dynamic scaling, P. C. Hohenberg and B. I. Halperin, Rev. Mod. Phys. 49, 435 (1977).

${ }^{39}$ We thank B. I. Halperin for discussions on this question.

${ }^{40}$ Strong disorder has been seen to modify the critical behavior at the 3D superfluid transition in the closely related system of ${ }^{4} \mathrm{He}$ absorbed in porous media. See, M. H. W. Chan, K. I. Blum, S. Q. Murphy, G. K. S. Wong, and J. D. Reppy, Phys. Rev. Lett. 62, 1950 (1988).

${ }^{41}$ A. T. Dorsey (unpublished) obtained (4.14).

${ }^{42}$ M. C. Marchetti and D. R. Nelson, Phys. Rev. B 41, 1910 (1990).

${ }^{43}$ The estimate for the width of the low-field fluid region, (5.1), ignored the effects of short-wavelength vortex-line fluctuations. Because the magnetic coupling is long range in the $z$ direction - with range essentially $\lambda_{\perp}$ for small $\gamma$ - the longwavelength tilt stiffness of the vortex lines will only apply for wavelengths greater than $\lambda_{\perp}$ and the vortex line is less stiff at short wavelengths. This will yield an extra contribution to the vortex-line fluctuations which will, however, only give displacements of order $\lambda_{1}$ in the final critical region where $\lambda_{z}=\Lambda_{T}$. Thus, the expression (5.1) remains valid in the $X Y$ critical regime. An exception to this is if the layers are so weakly coupled that $\lambda_{z}>\lambda_{1}^{2} / d$, in which case the fluctuations with wavelengths of order the layer spacing can play a role-and the system becomes quasi-2D. We will not investigate this regime in detail here, but just note that it is unlikely to apply sufficiently close to $T_{c}$ to have much effect on the melting phase boundary even for parameters appropriate for Bi-Sr-Ca-Cu-O.

${ }^{44}$ D. S. Fisher, Phys. Rev. B 22, 1190 (1980).

${ }^{45}$ M. V. Feigel'man, V. B. Geshkenbein, A. I. Larkin, and V. M. Vinokur, Phys. Rev. Lett. 63, 2303 (1989). These authors did not consider the effects of dislocations in the vortex lattice on scales larger than $L_{P}$.

${ }^{46}$ M. V. Feigel'man and V. M. Vinokur, Phys. Rev. B 41, 8986 (1990).

${ }^{47}$ E. Brezin, D. R. Nelson, and A. Thiaville, Phys. Rev. B 31, 7124 (1985).

${ }^{48}$ In a vortex lattice, the phonon fluctuations make evaluation of the correlations, $G_{\mathrm{VG}}(\mathbf{r})$, subtle; a recent claim [M. A. Moore, Phys. Rev. B 39, 136 (1988)] that there is no long-range order is based on the incorrect handling of the long-wavelength fluctuations. See A. Houghton, R. A. Pelcovits, and A. Sudbo, Phys. Rev. B 42, 906 (1990).

${ }^{49}$ D. A. Huse and D. S. Fisher, J. Phys. A 20, L997 (1987); D. S. Fisher and D. A. Huse, ibid. 20, L1005 (1987).

${ }^{50}$ For example, M. Kardar and Y.-C. Zhang, Phys. Rev. Lett. 58, 2087 (1987); D. S. Fisher and D. A. Huse (unpublished).

${ }^{51}$ Y. Yeshurun, A. P. Malozemoff, and F. Holtzberg, J. Appl. Phys. 64, 5797 (1988), and references therein; see also A. P.
Malozemoff and M. P. A. Fisher, Phys. Rev. B 42, 6784 (1990).

${ }^{52}$ The vortex-glass transition in the low-dimensional "toy" model studied in Ref. 11 also exhibits isotropic scaling behavior.

${ }^{53}$ A. T. Dorsey, M. Huang, and M. P. A. Fisher (unpublished).

${ }^{54}$ A. Houghton and M. A. Moore, Phys. Rev. B 38, 5045 (1988), have performed a static $6-\varepsilon$ expansion. The dynamical exponent $z$ can be obtained to $O(\varepsilon)$ from the static exponents, provided van Hove theory holds to leading order, as it does for the Ising spin glass; see A. Zippelius, Phys. Rev. B 29, 2717 (1984).

${ }^{55}$ With $v_{0}=\frac{2}{3}$ there will be logarithmic corrections to some quantities due to the marginal irrelevance of the disorder in this case. With the expected $v_{0}$ slightly bigger than $\frac{2}{3}$, the disorder is irrelevant at $H=0$ and the width of the vortex-glass critical regime will decrease slightly more rapidly than $\left[T_{c}-T_{G}(H)\right]$ as $H \rightarrow 0$.

${ }^{56}$ See, for example, B. W. Morris, S. G. Colbourne, M. A. Moore, A. J. Bray, and J. Canisius, J. Phys. C 19, 1157 (1986); S. Jain and A. P. Young, ibid. 19, 3913 (1986).

${ }^{57}$ M. P. A. Fisher, P. B. Weichman, G. Grinstein, and D. S. Fisher, Phys. Rev. B 40, 546 (1989).

${ }^{58}$ The continuum gauge glass was studied by J. A. Hertz, Phys. Rev. B 18, 4875 (1978).

${ }^{59} \mathrm{~J}$. Villain, in Ill-Condensed Matter, edited by R. Balian et al. (North-Holland, Amsterdam, 1979), p. 521.

${ }^{60}$ T. K. Worthington, F. H. Holtzberg, and C. A. Field, Cryogenics 30, 417 (1990).

${ }^{61}$ See, e.g., M. R. Beasley, J. E. Mooij, and T. P. Orlando, Phys. Rev. Lett. 42, 1155 (1979).

${ }^{62}$ Inclusion of quantum fluctuations gives a nontrivial $T=0$ quantum transition, as $H$ is increased, from $2 \mathrm{D}$ vortex glass to insulator (Fermi or Bose glass), as has been examined by $\mathrm{M}$. P. A. Fisher, Phys. Rev. Lett. 65, 923 (1990). Moreover, when one approaches the $T=0$ vortex-glass phase, quantum variable range hopping of vortices may dominate the resistivity, instead of the thermal processes we discuss here.

${ }^{63}$ See, L. Krusin-Elbaum, R. L. Greene, F. Holtzberg, A. P. Malozemoff, and Y. Yeshurun, Phys. Rev. Lett. 62, 217 (1989), and references therein.

${ }^{64}$ G. J. Dolan, F. Holtzberg, C. Field, and T. R. Dinger, Phys. Rev. Lett. 62, 2184 (1989).

${ }^{65}$ For example, S. E. Inderhees, M. B. Salamon, N. Goldenfeld, J. P. Rice, B. G. Pazol, D. M. Ginsberg, J. Z. Liu, and G. W. Crabtree, Phys. Rev. Lett. 60, 1178 (1988).

${ }^{66}$ T. K. Worthington, Y. Yeshurun, A. P. Malozemoff, R. M. Yandfrofski, F. H. Holtzberg, and T. R. Dinger, J. Phys. (Paris) Colloq. 50, C8-2093 (1989).

${ }^{67}$ R. H. Koch (unpublished).

${ }^{68}$ M. C. Marchetti and D. R. Nelson, Phys. Rev. B (to be published).

${ }^{69}$ B. Oh, K. Car, A. D. Kent, M. Naito, M. R. Beasley, T. H. Geballe, R. H. Hammond, A. Kapitulnik, and J. M. Graybeal, Phys. Rev. B 37, 7861 (1988); T. K. Worthington, W. J. Gallagher, D. L. Kaiser, F. H. Holtzberg, and T. R. Dinger, Physica C 153-155, 32 (1987).

${ }^{70}$ D. R. Harshman et al. (unpublished).

${ }^{71}$ S. Martin. A. T. Fiory, R. M. Fleming, G. P. Espinosa, and A. S. Cooper, Phys. Rev. Lett. 62, 677 (1989). 